University of South Florida

DIGITAL COMMONS Digital Commons @ University of @ UNIVERSITY OF SOUTH FLORIDA South Florida

3-1-2008

\title{
Testing the Impact of Personalized Feedback on Household Travel Behavior (TRAC-IT 2)
}

CUTR

Follow this and additional works at: https://digitalcommons.usf.edu/cutr_nctr

\section{Recommended Citation}

"Testing the Impact of Personalized Feedback on Household Travel Behavior (TRAC-IT 2)," National Center for Transit Research (NCTR) Report No. CUTR-NCTR-RR-2005-05, Center for Urban Transportation Research, University of South Florida, 2008.

DOI: https://doi.org/10.5038/CUTR-NCTR-RR-2005-05

Available at: https://scholarcommons.usf.edu/cutr_nctr/163

This Technical Report is brought to you for free and open access by the National Center for Transit Research (NCTR) Archive (2000-2020) at Digital Commons @ University of South Florida. It has been accepted for inclusion in Research Reports by an authorized administrator of Digital Commons @ University of South Florida. For more information, please contact digitalcommons@usf.edu. 


\title{
Testing the I mpact of Personalized Feedback on Household Travel Behavior \\ (TRAC-IT Phase 2)
}

\author{
Final Report
}

FDOT BD 549 WO 24

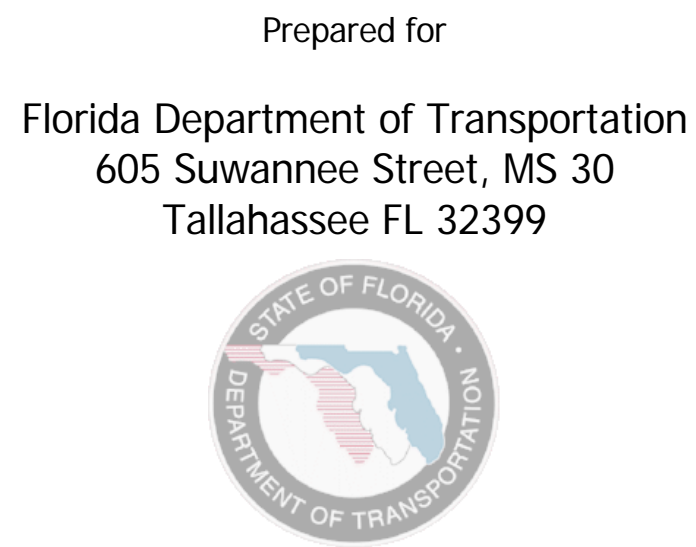

Prepared by

National Center for Transit Research (NCTR) at the Center for Urban Transportation Research (CUTR) University of South Florida

4202 E. Fowler Ave. CUT100

Tampa, Florida, 33620-5375

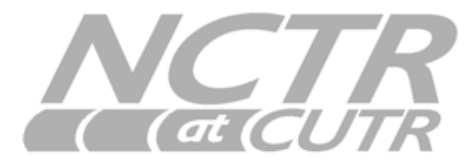

March 2008 


\section{Disclaimer}

The opinions, findings, and conclusions expressed in this publication are those of the author(s) who are responsible for the facts and accuracy of the data presented herein. The contents do not necessarily reflect the views or policies of the Florida Department of Transportation or the Research and Special Programs Administration. This report does not constitute a standard, specification, or regulation.

The report is prepared in cooperation with the State of Florida Department of Transportation and the U.S. Department of Transportation.

Patents Pending

Multiple patents on TRAC-IT system and components are pending, USF 2008. 


\begin{tabular}{|c|c|c|c|}
\hline $\begin{array}{l}\text { 1. Report No. } \\
\text { FDOT-BD 549, } \\
\text { RPWO\#24 } \\
\text { NCTR-77609-00 }\end{array}$ & \multicolumn{2}{|c|}{ 2. Government Accession No. } & 3. Recipient's Catalog No. \\
\hline \multirow{2}{*}{\multicolumn{3}{|c|}{$\begin{array}{l}\text { 4. Title and Subtitle } \\
\text { Testing the Impact of Personalized Feedback } \\
\text { on Household Travel Behavior (TRAC-IT Phase 2) }\end{array}$}} & \begin{tabular}{|l|} 
5. Report Date \\
March 2008
\end{tabular} \\
\hline & & & 6. Performing Organization Code \\
\hline 7. Author(s) & rbeau, S & J., and Georggi, Nevine L. & 8. Performing Organization Report No. \\
\hline \multirow{2}{*}{\multicolumn{3}{|c|}{$\begin{array}{l}\text { 9. Performing Organization Name and Address } \\
\text { National Center for Transit Research, Center for Urban } \\
\text { Transportation Research, University of South Florida } \\
\text { 4202 E. Fowler Avenue, CUT100 } \\
\text { Tampa, FL 33620-5375 }\end{array}$}} & 10. Work Unit No. (TRAIS) \\
\hline & & & $\begin{array}{l}\text { 11. Contract or Grant No. } \\
\text { DTRS } 98-9-0032\end{array}$ \\
\hline \multirow{2}{*}{\multicolumn{3}{|c|}{$\begin{array}{l}\text { 12. Sponsoring Agency Name and Address } \\
\text { Office of Research and Special Programs } \\
\text { U.S. Department of Transportation, Washington, D.C. } 20690 \\
\text { Florida Department of Transportation } \\
605 \text { Suwannee Street, MS 26, Tallahassee, FL } 32399\end{array}$}} & $\begin{array}{l}\text { 13. Type of Report and Period Covered } \\
\text { Final }\end{array}$ \\
\hline & & & 14. Sponsoring Agency Code \\
\hline \multicolumn{4}{|c|}{$\begin{array}{l}\text { 15. Supplementary Notes } \\
\text { Sponsored by a grant from the Florida Department of Transportation and the U.S. Department of } \\
\text { Transportation }\end{array}$} \\
\hline \multicolumn{4}{|c|}{$\begin{array}{l}\text { 16. Abstract } \\
\text { When presented with their household's travel patterns and practical ways to improve their trip } \\
\text { planning or even eliminate trips, more travelers could find new opportunities to use transit, } \\
\text { bicycling or walking, and do less solo driving. This three-phase study harnessed the exponential } \\
\text { growth of personal mobile electronic devices integrated with increasingly accurate global } \\
\text { positioning systems to develop a non-proprietary location aware information system (TRAC-IT). } \\
\text { The conceptual phase used a personal digital assistant equipped with GPS and developed a } \\
\text { rule-based expert system to collect travel data and generate travel suggestions. The second } \\
\text { phase created a Personal Travel Coach consisting of an enhanced rule-based expert system and } \\
\text { a real-time path prediction prototype. The third phase resulted in the first non-proprietary, } \\
\text { intelligent software system on GPS-enabled mobile phones to successfully track person } \\
\text { movements across all modes and also developed innovative analysis techniques such as } \\
\text { purpose and mode detection algorithms. Path prediction allows TRAC-IT to detect potential } \\
\text { incidents within the user's probable travel route and provide real-time travel advice to the } \\
\text { traveler before they reach the problem areas. The TRAC-IT system should advance the quality } \\
\text { and quantity of household multimodal data collected in travel surveys. The TRAC-IT } \\
\text { application's Personal Travel Coach components; the Expert System and Path Prediction } \\
\text { Prototype, give transportation professionals the next-generation location aware information } \\
\text { system they need to understand, plan, and influence travel behavior. Technical and policy- } \\
\text { based challenges, inherent with such innovative research, are also discussed. }\end{array}$} \\
\hline \multicolumn{2}{|c|}{$\begin{array}{l}\text { 17. Key Word } \\
\text { travel behavior survey, global } \\
\text { positioning systems, cellular } \\
\text { phones, travel feedback advice, } \\
\text { path prediction, route prediction } \\
\text { 19. Security Classif. (of this report) }\end{array}$} & \multicolumn{2}{|c|}{$\begin{array}{l}\text { 18. Distribution Statement } \\
\text { Available to the public through the National Technical Information } \\
\text { Service (NTIS), 5285 Port Royal Road, Springfield, VA 22161, (703) } \\
\text { 487-4650, http://www.ntis.gov/, and through the NCTR web site at } \\
\text { http://www.nctr. usf.edu/ }\end{array}$} \\
\hline $\begin{array}{l}\text { 19. Security Classif. } \\
\text { Unclassified }\end{array}$ & is report) & 20. Security Classif. (of this page) & \begin{tabular}{|l|l|} 
21. No. of Pages & 22. Price \\
125 & \\
\end{tabular} \\
\hline
\end{tabular}




\section{Acknowledgements}

This report is prepared by the National Center for Transit Research through the sponsorship of the Florida Department of Transportation (FDOT) and the U.S. Department of Transportation.

FDOT Project Manager:

Michael Wright, FDOT Public Transit Office

Project Team:

Philip L. Winters, Principal Investigator and TDM Program Director, CUTR

Co-Principal Investigators:

Sean Barbeau, Research Associate, CUTR

Nevine Labib Georggi, Research Associate, CUTR

Rafael Perez, PhD, College of Engineering, USF

Miguel Labrador, PhD, College of Engineering, USF

Computer Science and Engineering Students Team:

David Aguilar

Paola Gonzalez

Narin Persad-Maharaj

Samuel Rivera

Jeremy Weinstein

Tiffany Burrell

Dmitry Belov

Alfredo Perez

Diana Arteaga

Milena Sarmiento

The research team thanks the Sprint-Nextel Application Developer Program for its cooperation and the donation of phones and cellular service that were tested as part of this research project. 


\section{Executive Summary}

This final report is the second in a series to describe a joint collaboration between the Transportation Demand Management (TDM) Program at the Center for Urban Transportation Research (CUTR) at the University of South Florida (USF), the Computer Science Engineering (CSE) Department at the College of Engineering at USF. This three-phase study, sponsored by the Florida Department of Transportation (FDOT), harnessed the exponential growth of personal mobile electronic devices integrated with increasingly accurate global positioning systems (GPS) to develop a non-proprietary location aware information system (TRAC-IT). The TRAC-IT system should advance the quality and quantity of household multimodal data collected in travel surveys.

This three-phase study builds on previous research conducted by CUTR for the FDOT Research Center Office in 1999 (1). The project offered a practical exercise that led households to re-appraise their needs and rationale for travel. Specific suggestions aimed at use of public transit service, bike paths, trip chaining, ridesharing, and e-commerce options were provided based on specific travel patterns observed in the activity diaries. A weeklong travel diary recorded on a paper handout formed the basis of the personalized advice provided. The 1999 study demonstrated that providing personalized travel advice to households based on their travel habits did reduce vehicles miles of travel relative to the control group.

The first phase developed a travel data collecting tool that consisted of a personal digital assistant (PDA), a GPS device, and a wireless card. The GPS unit provided a means of tracking time, route, and speed, while the PDA provided a means of recording items such as mode, occupancy, and trip purpose. Phase 1 also designed a preliminary rule-based feedback advisory system (also referred to as the expert system) that generated tailored suggestions to improve household trip planning or even eliminate trips. The advice provided more information on other travel options such as transit, bicycling or walking, telecommuting, ridesharing and online shopping. TRAC-IT Phase 1 successfully tested the proof-of-concept of collecting and using data from mobile devices with TRAC-IT software and generated tailored feedback from the expert system for each user based on his/her household travel behavior over the entire test period (2).

The second phase, the focus of this report, produced a Personal Travel Coach consisting of an enhanced rule-based Expert System and a real-time Path Prediction Prototype. The Path Prediction prototype has the ability to potentially change travel behavior in real-time while the Expert System module focuses on long-term and sustained behavior change. The TRAC-IT application's Personal Travel Coach components, the Expert System and Path Prediction Prototype, gives 
transportation professionals the next-generation location aware information system they need to understand, plan and influence travel behavior.

The Expert System is a rule-based, automated system that analyzes individual and household travel behavior data represented as GPS and user-entered survey information and generates personalized feedback to the user. This feedback contains specific travel advice, such as carpooling or information about using alternate modes of transportation that could potentially save the user time and/or money. The TRAC-IT Expert System rules for this research project were developed by the TDM community for use in travel behavior change field studies (2). The rules are used to analyze trip data to identify inefficient travel behavior and construct meaningful feedback to users based on specific trips that the user recorded. The initial TRAC-IT system from Phase 1 was able to successfully upload the GPS and user-entered survey data collected by the mobile device to the server and provide automated suggestions, generated by the expert system, for more efficient transportation options based on the household travel behavior. Collected data could also be manually reviewed by transportation professionals at any time through access to the TRAC-IT server database where the trip data is stored. The expert system was designed to automate some of the trip analysis that a transportation demand management professional would normally perform.

Phase 2 also focused on the development of a Path Prediction module for the Personal Travel Coach which uses sophisticated real-time GIS analysis to predict a user's probable trip path and provides real-time alerts of any traffic incidents associated with predicted path. Path prediction records user travel behavior, predicts the most probable immediate trip route, looks ahead at each path of the route, and warns the user of any incidents detected along these paths. This information is delivered to the user immediately and alerts the user to take an alternate route before encountering the incident on their path.

The third phase of this research effort was dedicated to the design, development, and testing of the GPS-enabled cellular phone as a TRAC-IT unit. Phase 3 resulted in the first non-proprietary, intelligent software system on GPS-enabled mobile phones to successfully track person movements across all modes and also developed innovative analysis techniques such as purpose and mode detection algorithms. Path prediction allows TRAC-IT to detect potential incidents within the user's probable travel route and provide real-time travel advice to the traveler before they reach the problem areas. This TRAC-IT feature, when combined with the ubiquity of mobile phones, should increase the effectiveness of 511 information systems towards changing travel behavior by moving to an information push model instead of pull (i.e., user automatically receives notices instead of calling into the system to retrieve data) while ensuring only relevant information is sent to the user (i.e., location information is used to filter out irrelevant data). 
Phase 2 planned on investigating the impact of the feedback advice on household travel behavior. Though the testing of the effectiveness of feedback advice using a before and after survey was not accomplished, this leapfrog research project has made the following significant contributions:

1. The development of an enhanced Personal Travel Coach with a new and improved implementation that supports eight rules that analyze household and individual travel patterns before providing suggestions.

2. The redesign of the TRAC-IT travel data collection tool to include userfriendly wizards with minimal input from the participant. The PDA version was successfully transferred to a new cell phone version. The new software system (i.e., server and mobile phone software) that supports GPS-enabled mobile phones was developed and tested. This mobile phone version is the first software-only travel behavior data collection tool that can track a person's travel behavior regardless of mode (e.g., auto, bus, bike, and walk) by using a hardware device that most people already own and is always with them as opposed to previous systems that track vehicle (e.g., AVL for transit, Vehicle Navigation Systems for auto) or require proprietary devices dedicated to data collection.

3. It increased the depth and breadth of travel behavior data with minimal burden on the participant by automatically collecting route, travel time, time and day of week, and travel distance from GPS data while still collecting survey data input manually from the user for items such as purpose, mode of transportation, beginning and ending location descriptions, and occupancy.

4. It identified how to passively collect trip purpose through integration of GPS position data with local tax GIS records.

5. It identified how to passively collect mode of transportation data through a mode detection algorithm.

6. It introduced feedback in the form of email with a visual map to reach participants after they have finished recording their trip so participants can instantly confirm their trip details via a reply email before they forget.

7. It developed the Path Prediction prototype module of the Personal Travel Coach that is able to predict the users' travel paths based on their real-time location and their previous personalized travel behavior history. Path prediction allows the TRAC-IT system to detect potential incidents that lie within the user's possible travel route and push travel advice to the user in real-time via text or audio alerts to the mobile phone before they reach problem areas. This TRAC-IT feature should increase the effectiveness of 511 information systems towards changing travel behavior by moving to an information push model instead of pull (i.e., user automatically receives notices instead of calling into the system to retrieve data) while assuring only relevant information is sent to the user (i.e., location information is used to filter out irrelevant data). 
8. It developed an electronic orientation session that can be uploaded to the Internet, emailed as an attachment, or sent to a survey participant in a CDROM format. The advantage of the electronic session is that the survey participant can view it at their own convenience and their own pace. Also, they can repeatedly view it in case they need to refresh their memory on how to use TRAC-IT. If consent forms are electronically signed, the entire recruitment process of participants can be remotely conducted eliminating the time it would take both survey participants and researchers to conduct and attend live orientation sessions.

Once the TRAC-IT system is stabilized, the experimental testing outlined as prepared for this project should be carried out in the future as the potential benefits of impacting travel behavior by providing personalized feedback have proven effective in previous pilot studies. The following tasks must be accomplished before TRAC-IT is deployed and the experimental testing is conducted:

(1) Resolve remaining TRAC-IT technical software issues:

- Distance calculation - Software that handles distance calculation is an issue that occasionally results in erroneously large distance measurements. This problem should be resolved so distance-based expert system rules can provide more accurate advice.

- Database table locking - There is a problem with the TRAC-IT system during periods of intermittent connectivity between the mobile phone and server. If there is a communication failure (i.e., cellular network not available) during times when the TRAC-IT user tries to begin or end a trip, the database is left in a state of an exclusive lock for the last phone that attempted access. Once this lock occurs, no other phones can perform any functions, including log-in to the system and starting or stopping trip recording until a system administrator manually intervenes. This issue must be resolved to stabilize the system and always allow users to log in and record their trips.

- Server restarts - There exists a configuration issue that requires the TRAC-IT server to restart when new updates become available to protect the system. However, this update process interferes with the TRAC-IT software server and does not always allow the system to restart properly. This is one of the reasons users are occasionally prevented from logging in and recording trips. The server must be configured to schedule restarts at opportune times enabling the TRAC-IT software server to remain functional throughout this procedure. 
(2) Implement enhanced TRAC-IT features to resolve existing weaknesses and improve system performance:

- Trip data caching - The current version of TRAC-IT relies on always-on network connectivity in order to immediately transfer the results of a trip to the TRAC-IT server. This system design allows even low-end cell phones to use TRAC-IT since no on-board data storage for trips are required. However, there are many cases where data connectivity is lost to mobile applications due to lack of cellular coverage, roaming, or wireless interference. These issues account for the greatest loss in reported trip data for the current TRAC-IT system, necessitating an improvement to the system. To enhance the reliability of the TRAC-IT system, temporary on-board caching of trip data should be implemented in future versions. Temporary caching of trip data would allow TRAC-IT to remain operable even when network connectivity is interrupted and therefore is recommended as a critical module to be developed.

Although additional new features may not be critical to the implementation of experimental testing, it would greatly enhance the performance of the TRAC-IT system in general. Passive tracking is a feature that will optimize the number of reported trips by the TRAC-IT system and increase the validity of results. The number one usability issue reported by participants in the TRAC-IT 3 field tests was their trouble remembering to start recording trips using the TRAC-IT interface. However, participants did report that they did not have nearly as much difficulty remembering to carry the TRAC-IT mobile phone with them. Since the goal of TRAC-IT is for the software application to be installed on personal mobile phones, it is very likely that future participants carrying TRAC-IT on their own phone will always have the phone with them. Passive tracking, which does not require the user to actively start/stop recording trips, is a feature that will decrease the number of missed trips.

Future research should also examine linking TRAC-IT to 511 or other forms of real-time advanced traveler information systems to push information to the traveler based on where they are currently and where they are likely to be going. Such real-time information could include traffic incidents, dynamic congestion pricing, managed lane pricing, highly-targeted commercial advertising (when appropriate), transit scheduling changes, and other useful data.

The research team attempted to gain real-time access to traffic information through Traffic.com in Tampa Bay. However, issues concerning intellectual property rights and data ownership failed to provide access. Now that Navteq has purchased Traffic.com, it may be possible to re-open a dialog with Traffic.com to test TRAC-IT using real-time speed sensors on Tampa Bay highways. Alternately, other cities with different types of advanced traveler information systems in areas such as South Florida could be evaluated for the possibility of linking those 
systems to TRAC-IT. Future enhancements for incident detections and alerts could include relaying driving directions for alternate routes and verbal feedback to the user as an improvement over the current text message that simply alerts the user of an incident. Since TRAC-IT is not restricted to a vehicle, such information could reach individuals before they are traveling on multiple modes of transportation such as walking, biking, and transit.

Finally, the applications of TRAC-IT are not limited to travel behavior change. This tool could help evaluate the travel patterns of various market segments or evaluate policy decisions. For example, TRAC-IT could be used to evaluate the impacts of various land use proposals such as transit-oriented development on transit use, green house gases, and mobility. TRAC-IT provides a simple method to collect any type of location-based data. Consequently, it might be useful to link mobile phones to alternate types of devices, such as carbon-dioxide sensors, or to vehicles to collect other types of data via the mobile phone. 


\section{Table of Contents}

Chapter One - I ntroduction ...................................................................................... 1

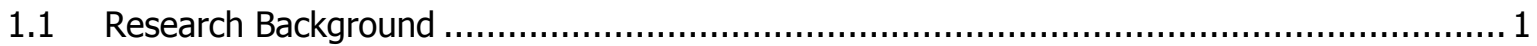

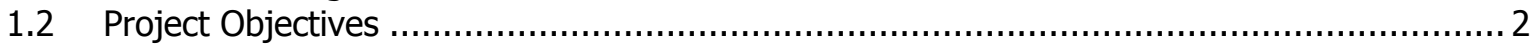

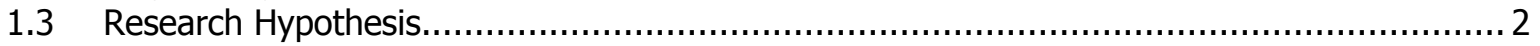

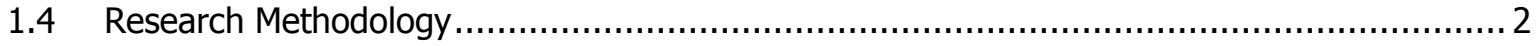

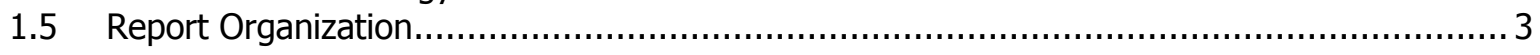

Chapter Two - Literature Review.......................................................................................... 4

2.1 Sample Size of Travel Survey...................................................................... 4

2.2 Travel Behavior Change Techniques................................................................. 7

2.2.1 Overview of TRAC-IT Phase 1 ................................................................. 7

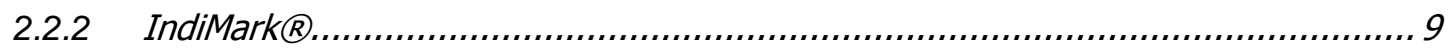

2.2.3 Individualized Marketing Demonstration Program (IMDP) ............................... 10

2.2.4 TravelSmart Australia ................................................................................ 12

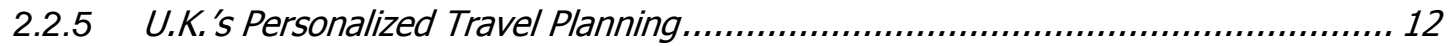

2.2.6 Japan's Travel Feedback Program .......................................................... 13

2.3 Types of Feedback and Delivery methods ........................................................... 14

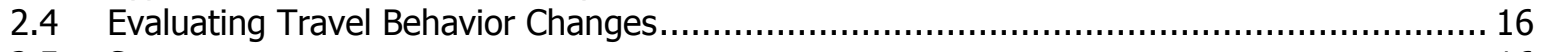

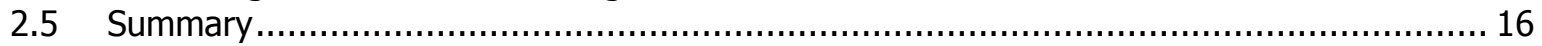

Chapter Three - Personal Travel Coach: Expert System Module .................................. 18

3.1 TRAC-IT Expert System Concept................................................................... 18

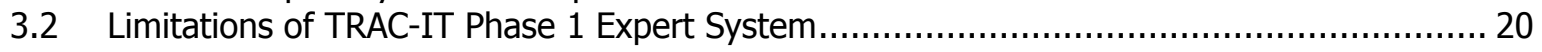

3.3 Design of TRAC-IT Phase 2 Expert System ........................................................ 24

3.4 TRAC-IT Phase 2 Expert System Module Implementation ......................................... 27

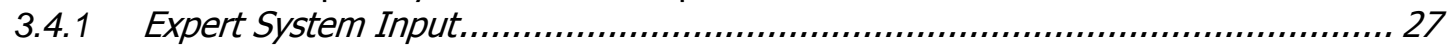

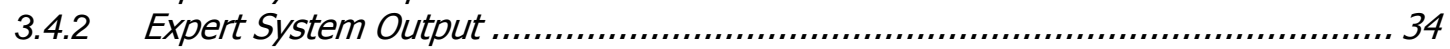

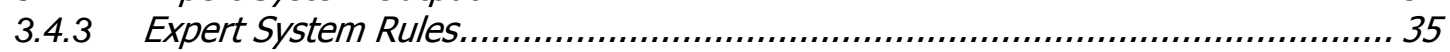

Chapter Four - Personal Travel Coach: Path Prediction Prototype............................... 41

4.1 Real-time Path Prediction and Traffic Incident Alerts.............................................. 42

4.1.1 Differences between Path Prediction and Expert System ................................. 43

4.1.2 Differences between Path Prediction and 511 .............................................. 44

4.1.3 Differences between Path Prediction and Other Algorithms ............................. 44

4.2 Methodology used for Path Prediction Prototype....................................................... 46

4.2.1 The Path Maker Algorithm ...................................................................... 46

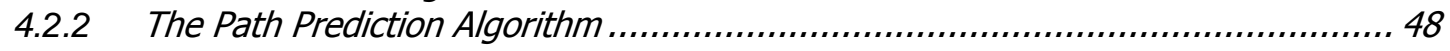

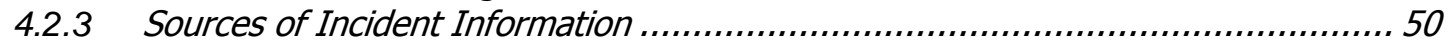

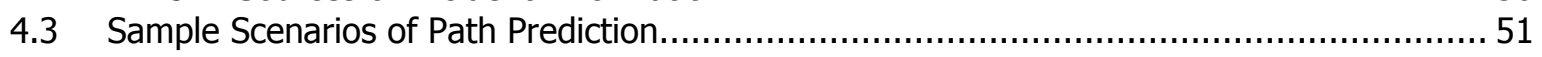

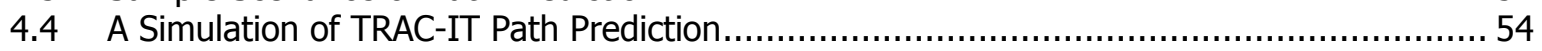

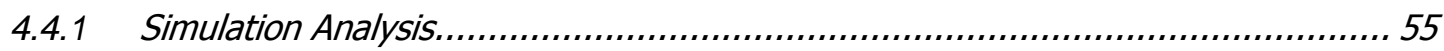

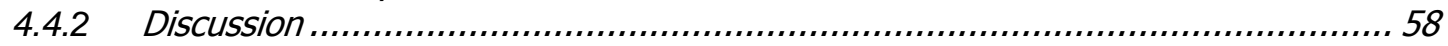


Chapter Five - TRAC-IT Phase 2 Survey I mplementation........................................... 60

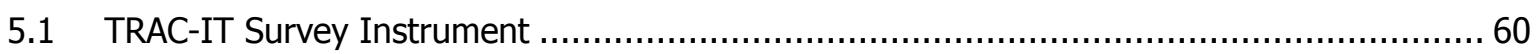

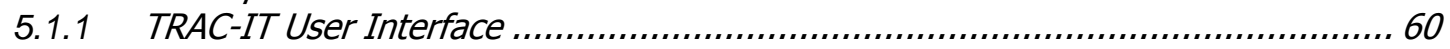

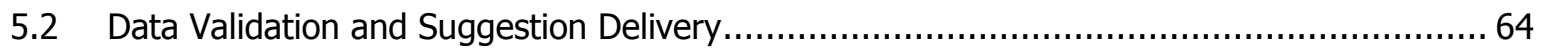

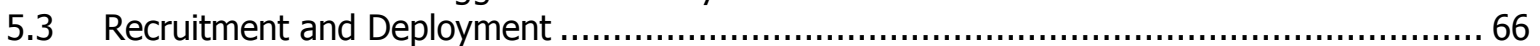

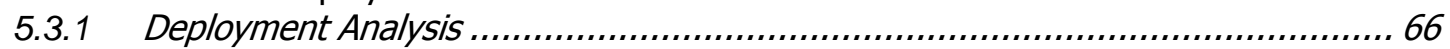

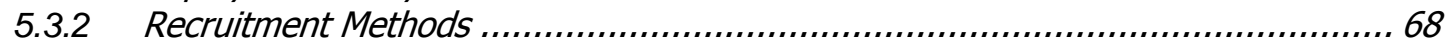

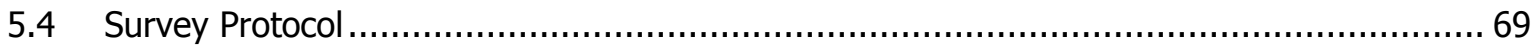

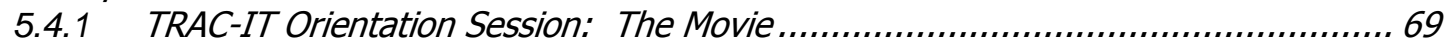

Chapter Six - Survey Analysis and Findings........................................................... 74

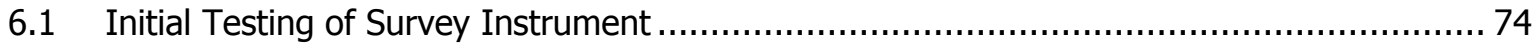

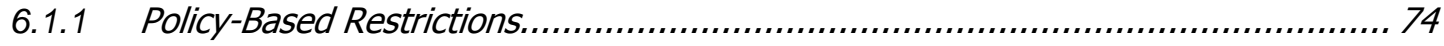

6.1.2 Rapidly Progressing Technology .................................................... 75

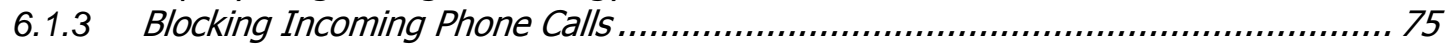

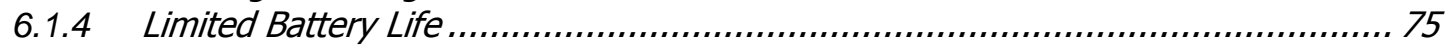

6.1.5 Privacy Concerns for Passive Tracking Feature.......................................... 76

6.1.6 Users Forgetting to Record Trips ...................................................... 76

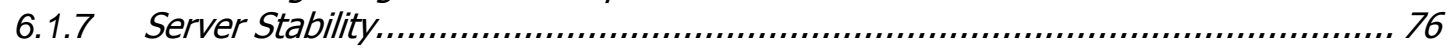

6.1.8 TRAC-IT Mobile Phone Software Issues................................................ 77

6.2 Expert System Feedback from TRAC-IT 3 Field Tests .............................................. 77

Chapter Seven - Conclusions and Recommendations............................................... 80

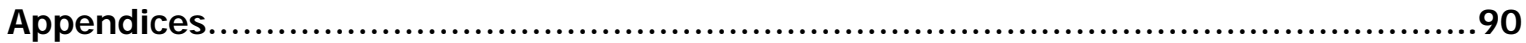




\section{List of Figures}

Figure 1 - Expert System Feedback: an illustration of sample text messages .......................... 19

Figure 2 - Conceptual Flowchart of the TRAC-IT Expert System......................................... 20

Figure 3 - Transact-SQL Implementation Module of the TRAC-IT Phase 1 Expert

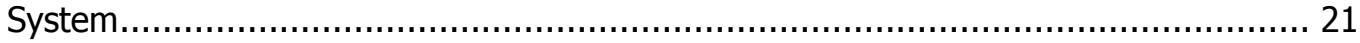

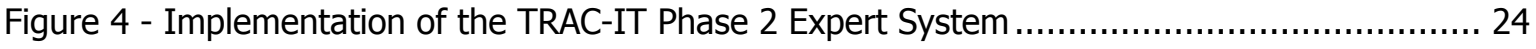

Figure 5 - Enhanced Database Schema for TRAC-IT 2 Expert System ................................... 29

Figure 6 - Personal Travel Coach Conceptual Diagram .................................................. 30

Figure 7 - Screenshot of a Simulation of TRAC-IT GPS-enabled Mobile Phone

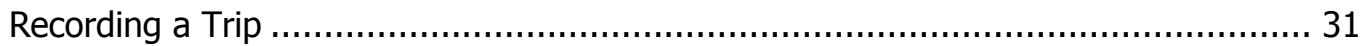

Figure 8 - TRAC-IT Menu for Modes of Transportation....................................................... 32

Figure 9 - TRAC-IT Mobile Application allows User-entered Location Descriptions to Assist in Personalizing Expert System Feedback ............................................ 34

Figure 10 - Path Prediction Prototype - an illustration of text message alerts .......................... 41

Figure 11 - Comparison between the Two Components of the Personal Travel

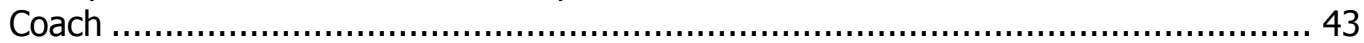

Figure 12 - Flowchart for Path Maker Algorithm........................................................... 47

Figure 13 - Polygon Network Topology for User Recorded Trips ........................................ 48

Figure 14 - Flowchart of TRAC-IT Path Prediction Algorithm.............................................. 49

Figure 15 - Real-time Trip Data Fully Contained in a Single Polygon .................................. 52

Figure 16 - Real-time Trip Data Partially Intersect a Single Polygon .................................... 52

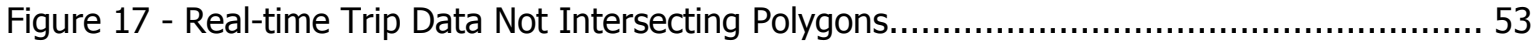

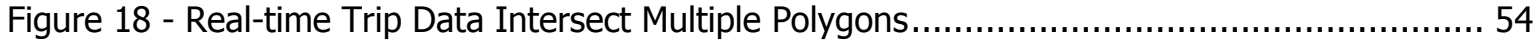

Figure 19 - Real-time Trip Data Fully Contained Within Multiple Polygons ............................. 54

Figure 20 - Iteration 1 of Path Prediction Algorithm Simulation .............................................. 56

Figure 21 - Iteration 33 of Path Prediction Algorithm Simulation ........................................ 56

Figure 22 - Iteration 40 of Path Prediction Algorithm Simulation Showing First

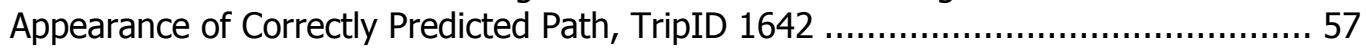

Figure 23 - Iteration 51 of Path Prediction Algorithm Simulation ..................................... 57

Figure 24 - Iteration 56 of Simulation Showing a Single Path, TripID 1642, Correctly Predicted Destination......................................................................... 58

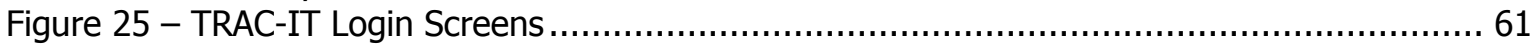

Figure 26 - Begin Trip and Current Location Screens..................................................... 62

Figure 27 - Menu of Transportation Modes for Selection by Participant ..............................6 64

Figure 28 - Trip Information and Feedback as an Outlook Meeting Invitation ..........................6 65

Figure 29 - TRAC-It Orientation Session Played as a Movie ............................................... 70

Figure 30 - A Collection of Screenshots from TRAC-IT Mobile Application ............................ 73 


\section{List of Tables}

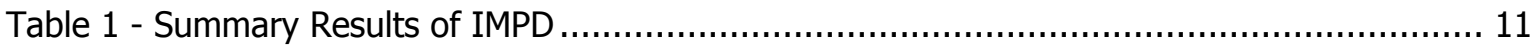

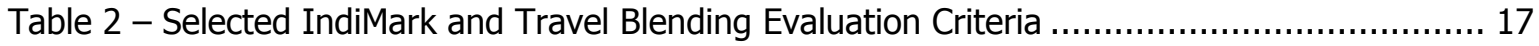

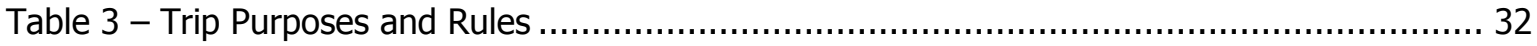

Table 4 - Sample Screenshots of the Flash Animations of TRAC-IT .................................... 71

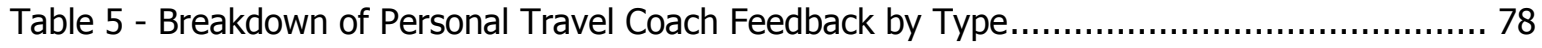




\section{Chapter One - Introduction}

\subsection{Research Background}

Transportation demand management (TDM) strategies are designed to motivate people to modify their travel choices, particularly the drive-alone preference. TDM strategies and policies seek to increase the transportation system efficiency and achieve specific objectives such as reduced traffic congestion, road and parking cost savings, increased safety, and improved mobility for non-drivers, energy conservation, and pollution emission reductions. Marketing these strategies to the consumer in an effective way that results in behavioral changes necessitates a sustained understanding of how, when, where, and why people travel. Therefore, accurate, timely, and comprehensive data are vital in understanding/influencing the consumer. Data collection processes have made use of new and emerging technologies that help refine the task of monitoring/measuring the behavior to be modified.

The research focuses on using innovative technology to better understand and pattern household travel behavior for the purposes of educating, promoting, and encouraging households to utilize other alternatives to driving in general and to driving alone in particular. The scope of Phase 1 called for preliminary development and testing of a portable unit consisting of a personal digital assistant (PDA), a global positioning system (GPS) device, and a wireless card, allin-one unit nicknamed TRAC-IT (2). Phase 3 in this research effort is dedicated to the design, development, and testing of the GPS-enabled cellular phone as a TRAC-IT unit. In Phase 2, the research team sought to investigate and analyze the impact of the travel feedback behavior change system on household travel behavior using the smart cell phone application developed in Phase 3.

This research builds on a 1999 study conducted by CUTR for the Florida Department of Transportation (FDOT) Research Center Office (1). The project offered a practical exercise that led households to re-appraise their needs and rationale for travel. Specific suggestions aimed at the use of public transit service, bike paths, trip chaining, ridesharing, and e-commerce options were provided based on specific travel patterns observed in the activity diaries. A weeklong travel diary formed the basis of the personalized advice provided. The study demonstrated that households provided with personalized advice did reduce vehicles miles of travel relative to the control group.

The research demonstrated a statistically significant difference between the travel behavior of people who receive travel reduction suggestions and those who do not receive such suggestions. However, it was not determined if the provision of generic suggestions (such as share a ride, ride the bus, etc.) had less or more impact on travel patterns than provision of customized suggestions based on 
household or individual travel behavior. One of the research conclusions was that efficient methods of collecting such data and providing travel suggestions had not been developed, a necessary step before a large-scale implementation of this procedure can be attempted.

At the time the study was completed (1999), Cleland and Winters suggested that a GPS-based system, in conjunction with, or in addition to, an Internet-based system of recording travel modes and providing feedback, would be implementable on a much larger scale. They recommended that thorough testing of such a system be completed on a sample large enough to allow for statistical validity and for projections to larger populations. Since the study provided reasonable confidence that a system of this nature would produce positive results, future research was recommended to find out whether the system would produce enough reduction in travel to merit the cost of implementation. Phase 1 of this research study developed a prototype to research the potential application of these recommendations.

\subsection{Project Objectives}

The objectives for this project were to (1) fine tune the trial expert advice system designed in Phase 1 by testing the system on a larger sample of households and expand its capability to provide customized advice, and (2) quantify changes in travel behavior patterns after providing personalized travel advice to encourage individuals to choose a mix of travel choices to satisfy their travel needs rather than repeatedly drive alone.

\subsection{Research Hypothesis}

The research hypothesis is that if household members were to recognize patterns of their daily travel behavior and offered practical ways to improve their trip planning or even eliminate trips, they would more often select the use of transit, bicycling or walking, and do less solo driving.

\subsection{Research Methodology}

Using the electronic travel diary system developed in Phase 1 for PDAs as a starting point and expanding it to a Personal Travel Coach on a smart phone platform under Phase 3, this project proposed to collect baseline travel behavior data from a sample of households over an extended period (2 - 4 weeks). Under the enhanced TRAC-IT system, the data collected are instantly transferred to a database that comprises part of the Personal Travel Coach. The data are analyzed and appropriate travel suggestions are generated. The initial plan was to provide customized travel suggestions to the experimental group and not to a control group. Another round of activity-based travel data was to be collected using the 
handheld device after providing the suggestions. Analysis of travel behavior changes before and after travel advice was analyzed and presented.

\subsection{Report Organization}

The next chapter presents a literature review, including an overview of Phase 1 of this research study, travel behavior change techniques, and types of feedback delivery methods. Chapter 3 presents the first component of the Personal Travel Coach, the Expert System, and Chapter 4 presents the second component, the Path Prediction prototype. Chapter 5 reports on the survey instrument, protocol and recruitment, and deployment of the TRAC-IT Personal Travel Coach. The sixth chapter reports survey analysis including test results of feedback. The last chapter reports on study conclusions and future research needs. 


\section{Chapter Two - Literature Review}

This chapter summarizes the literature review conducted by the research team to determine effective marketing and evaluation techniques for travel blending. The research team looked into travel surveying studies to determine sample size and survey duration as discussed in the first section of this chapter. The second section is a review of Phase 1 of this research series highlighting the findings and recommendations of that project. Section 3 reviews five travel blending techniques used both abroad and in the United States. The fourth section reviews different marketing techniques in providing advice to households and best practices that encourage travel behavior changes. Sections 5 and 6 identify rules used by others to determine the type of advice provided and various methods used to deliver such advice. Section 6 determines the appropriate performance measures used to evaluate travel behavior changes. Last, a discussion of the findings of the literature review is presented.

\subsection{Sample Size of Travel Survey}

A review of the literature found a wide range of sample sizes and design for comparing the relative effectiveness of travel behavior change programs. The following section summarizes the approach and sample size requirements for TRAC-IT based on best available data.

O'Fallon and Sullivan note several pitfalls with respect to sample sizes in measuring the effects of travel behavior change programs (3). In particular, they note that the evaluations of such programs are often confounded by biases in the selection of participants exposed to the treatment in the travel behavior program (e.g., Indimark's focus on individuals willing to change) and by changes in external factors (e.g., changes in gas prices between before and after surveys). Determining the appropriate sample size to account for known variability will permit the establishment of reasonable confidence intervals around the change in behavior.

One of the factors for determining accurate sample size estimates is the variability of the dependent variable. For a higher degree of variability, a larger sample size is required. Determination of the sample size is based on the specification of the smallest effect to be detected by the research team, the Type I (rate of false positives) and Type II error rates (rate of false negatives), and the design of the study.

The required sample size for hypothesis testing in before and after surveys is given by (4): 


$\begin{array}{ll} & \frac{2\left(z_{a}+z_{\beta}\right)^{2}\left(\sigma^{2}\right)}{\delta^{2}} \\ \text { Where } & \\ n= & \text { required sample size } \\ \mathrm{a}= & \text { the probability of making a Type I error } \\ \beta= & \text { the probability of making a Type II error } \\ \mathrm{s}= & \text { the standard deviation of the parameter to be tested } \\ \delta= & \text { the required difference in the parameter to be tested }\end{array}$

Sample size requirements and costs can be reduced by moderating the changeability of the dependent variable using several approaches. First, a survey design where the same people are measured before and after travel advice is provided will not require sample sizes as large as repeated cross-sectional surveys. Sample size requirements also decrease if the travel behavior data can be collected over longer periods of time (week vs. day) as the variability declines. Finally, measuring travel behavior averaged across all people in a household would require lower sample sizes by further decreasing the variability.

The selection of vehicle trips as the dependent variable correlates closely with the goals of congestion reduction strategies -- reduce vehicle miles of travel, decrease air pollution, decrease the need for parking -- and is generally proportional to the desired result. Alternative dependent variables such as changes in single occupant vehicle (SOV) share or average vehicle ridership (AVR) have disadvantages. SOV share misses the benefits of moving from one non-SOV mode to another where the switch may actually reduce traffic volumes but not change SOV share of vehicle trips (e.g., carpool to transit). The reduction in vehicle trips is distorted when using AVR as the dependent variable due to the non-linear relationship between AVR and vehicle trips. For example, increasing AVR by 0.25 from 1.10 to 1.35 persons per vehicle would require a reduction of 17 vehicle trips per 100 people. The same increase (0.25) for a worksite with an AVR of 1.50 to 1.75 would only require a reduction of 9 vehicle trips per 100 people. The selection of vehicle trips as the dependent variable also influences the sample size. As Richardson noted, larger sample sizes are required to detect changes in distance traveled than in vehicle trips due to the larger variability of mileage versus trips.

As previously mentioned, in the 1999 research study conducted by CUTR, specific suggestions aimed at use of public transit service, bike paths, trip chaining, ridesharing, and e-commerce options were provided based on specific travel patterns observed in the activity diaries (1). Test and control groups completed two 7-day travel diaries that formed the basis of the testing the personalized advice provided. That study found the average number of weekly trips per household in the control group was 28.38 with a standard deviation of 5.03. Using these values to estimate sample size and assuming that $a$ and $\beta$ equal $5 \%$ 
(one-tailed) and $\delta=10 \%$ of the mean, then the required sample size is about 70 households.

These sample size reduction strategies can also make the survey more difficult to collect. As Richardson points out, panel data are more difficult to obtain than cross-sectional data because they are dependent on participants completing at least two rounds of surveys (4). Weekly surveys are more burdensome than daily activity diaries when collected via paper surveys. The value of using GPS to improve data quality and minimize respondent burden has been demonstrated. For example, the Federal Highway Administration (FHWA) conducted a pilot experiment in which one vehicle in each of 100 households in the Lexington, $\mathrm{KY}$ area was fit with a GPS unit and a small hand-held computer (5). Objectives of this FHWA research project, focused on analyzing day-to-day variability in travel behavior using the GPS-based data set, were to:

- Explore day-to-day variability in travel behavior with respect to selected variables such as trip chaining, departure time, trip frequency, and path selection.

- Compare day-to-day variability found in the GPS-based data set against that reported in the literature to assess the potential benefits and pitfalls associated with using a GPS-based data collection methodology.

- The devices tracked the movements of individual vehicles over six days. Some were tracked as along as eight or nine days, while minimizing respondent burden. In addition, the GPS-based survey is presumably more accurate than traditional personal recall surveys because one may forget to report short, infrequent trips that occur during the day. The GPS provides the added benefit of capturing route choice, path, and speed information that is typically very difficult to collect in a traditional personal recall survey.

The analysis showed that if the day of the week were controlled for when analyzing variability across multiple days, then the degree of variability measured is lower. It was also found that the longer the period of observation, the greater the degree of variability observed. Both of these findings are consistent with the literature, showing that GPS-based travel data sets are capable of offering plausible and reasonable inferences on travel characteristics. The analysis of the survey showed that there was considerable variability in travel behavior across multiple days. In general, the greatest stability was found to occur in three variables that represent temporal events - first departure from home, final arrival at home, and final departure from work.

Although the FHWA study did not take full advantage of the capabilities of GPS, it concluded that GPS-based travel data sets provide multiple days of travel information with plausible measures of day-to-day variability. It also concluded that GPS travel surveys have the potential to offer detailed route choice, spatial 
location, and travel itinerary information not available in other travel survey data sets.

A study by Schlich (2001) discusses how attempts to mitigate traffic congestion in a way that produces less environmental impact can succeed (6). The answer is only if the needs and desires of users are well understood. The more variable and complex the behavior is, the more flexible the solutions have to be. The one basic condition necessary in patterning variability is to observe the behavior over a long period, but how long? Schlich addressed this question by comparing the measured variability of activities of three randomly chosen persons for different durations. From the analysis, he determined that the periods always start with the first reported day and cover one to six weeks. Therefore, he concluded that empirical surveys about travel behavior research should cover a period of at least two weeks.

TRAC-IT will capture variability in travel characteristics collected for both individual and household, with little input from participant from 2 to 4 week periods.

The research team postulated that efforts to minimize the need for user input via smart cell phone tool, effectively becoming a smart diary, would ease respondent fatigue and thus allow extended periods of data collection. Much effort was spent on TRAC-IT's design to minimize the need for user input through the adoption of

graphical user interface (GUI) wizards (e.g., change of mode, frequently visited locations, and quick stops) (2).

Furthermore, the research team sought to enhance the value of the TRAC-IT application. The team devising a prototype means to push real time traffic information to them based on their location and expected travel path. The expected travel path was based on a travel prediction algorithm developed specifically for this project. More information on this path prediction element will be provided in Chapter 4.

\subsection{Travel Behavior Change Techniques}

Other researchers, primarily international, have sought to affect travel behavior change through the provision of personalized travel information in a variety of formats and applied to particular market segments and/or modes. The following summarizes these various approaches.

\subsubsection{Overview of TRAC-IT Phase 1}

The TRAC-IT unit in Phase I was designed to collect comprehensive individual trip data including start time, end time, origin, destination, travel speed, trip route, and trip distance with minimal input from participants (2). Limited field-testing was conducted to validate that the technology works. The project also developed 
a preliminary personalized feedback system that provides suggestions and encourages participants to utilize other modes than the drive-alone option. These suggestions were sent to participants after the trip data had been transferred from the TRAC-IT unit into a database developed for Phase 1 to pattern travel behavior and generate appropriate feedback to participants. The research team sought to develop a system that automatically collected travel behavior data at the personlevel (rather than vehicle-level) and provided personalized feedback based on the trip data collected by TRAC-IT units from participants and their households. For the purposes of Phase 1, the participants were identified as case studies since the goal of the field test was to make sure the prototype worked under various scenarios representing different modes of travel and varied household dynamics. These scenarios included:

- two-income household, two adults, two-car ownership, and one child under driving age

- single-income household, one female adult, zero-car ownership

- single-income household, two adults, one car and one bicycle, and three children under driving age

- single-income household, two adults, two-car ownership and one child under driving age (trips collected from one household member only)

Each adult in each household was provided with a TRAC-IT unit to record his or her travel behavior. Before participants were given their TRAC-IT units, each participating household was identified with a unique number, and each adult in the household was issued a user identification number. In addition, to reduce data input burden, a user profile was created in the PDA with the person's home and work addresses pre-entered.

The three primary requirements set forth to demonstrate the feasibility of the TRAC-IT prototype were:

- Improve the quantity of data collected

Through the GPS component, TRAC-IT was able to collect and record the location of the traveler, travel path, and speed. The PDA component was able to record GPS information as well as collect data on travel purpose and occupancy information. Algorithms developed specifically for this project to reduce nonessential data were developed to simplify the analysis. These algorithms were applied to the PDA component of the TRAC-IT unit. Finally, the system demonstrated the ability to record travel for all major modes (auto, transit, bike and walk) to provide a richer and deeper view of travel behavior.

- Improve the data quality

The team found that TRAC-IT's design minimized the need for user inputs through the adoption of GUI wizards (e.g., change of mode, frequently visited locations, and quick stops, or the ability to easily indicate that the starting 
location of a new trip is the same as the ending location of the previous trip). The development of Smart Diary components reduced respondent fatigue (allowing for longer periods of data collection) and improved data quality by populating the fields based on previous patterns by performing complicated realtime data processing and analysis. The automated aspects of TRAC-IT gathered completed data because of the development of wizards to capture quick stops, changes of mode, etc. The richness of the data collected via TRAC-IT includes the ability to collect and analyze multimodal travel behavior and patterns at the household level. Since data could be synched directly into a database, TRAC-IT eliminated the errors associated with manual data entry.

- Analyze travel behavior at the individual and household levels

The TRAC-IT system was able to successfully upload the data to the server and provide suggestions for more efficient transportation options based on the household travel behavior. The basic feedback advisory system, consisting of only the Expert System, was designed to test the ability of collecting and using the data. The TRAC-IT Expert System then generated feedback for each user based on his/her household travel behavior over the entire test period.

It was concluded that the TRAC-IT Expert System works. However, more rules need to be added and others need to be more descriptive and less general. Phase 2 sought to enhance TRAC-IT and test the GPS-enabled cell phone on a larger sample of households and measure the changes in household travel behavior after providing tailored travel advice using the trial expert system developed in Phase 1.

\subsubsection{IndiMark ${ }^{\circledR}$}

IndiMark is generally used for direct marketing of public transportation but has also been applied to encourage use of walking and cycling (7). It is also described as dialogue marketing because it aims to establish a dialogue with the participants. The process has seen successful implementation in Europe and Perth, Australia (8).

According to Socialdata Australia Pty Ltd, IndiMark was used effectively to reduce reliance on private cars in more than 50 projects with over 300,000 participants in Europe, the United States, and Australia. The achievements of IndiMark reflect a sustained reduction in car trips associated with a relative reduction in car kilometers traveled. Evaluations of large-scale projects have shown an average relative reduction in car kilometers traveled by up to 19 percent in Germany, 17 percent in Australia, and 13 percent in Sweden. In addition, IndiMark offers economic benefits that far exceed its costs and so provides free reductions in fossil fuel consumption and related emission. 


\subsubsection{Individualized Marketing Demonstration Program (I MDP)}

The Individualized Marketing Demonstration Program (IMDP) is a pilot program developed to test the effects of individualized marketing on public transportation ridership trends closely following the Indimark model. The FTA's IMDP focuses on personalized, individual marketing of potential commuters who might consider using public transit but need more information. Utilizing a dialog-based technique for promoting the use of public transport, the program provides targeted, personalized, customized marketing tailored for individuals that are most likely to change their travel behavior. Based on a single day diary, the first IndiMark U.S. pilot project in Portland, Oregon, reported a reduction in car travel of 8 percent and an increase in travel by environmentally friendly modes of 27 percent (9). The one-year evaluation of the pilot's success began in September 2003, and Portland officials have indicated that if the results hold firm, they will consider replicating the process throughout the city.

IMDP involves four main stages, as summarized in the following list, and includes rewards or incentives for participants.

- Contact - all households are contacted by mail and phone. A short survey is used to determine if they are regular or extensive users of environmentally friendly modes $(R)$, are not interested in changing $(N)$, or are interested (I).

- Motivation - requests from the (R) and (I) groups are responded to.

- Information - the (R) and (I) participants select the information they want which can include maps, timetables, and further information on particular modes. Individuals in the (R) group may also receive rewards for their use of Environmentally Friendly Modes (EFM).

- Convincing - consultation phone calls and home visits on request are conducted, with selected households in group (I) receiving tickets to use on public transport for a limited period.

The concept is one of measuring behavior change due to personalized marketing efforts. It includes a before survey (self-administered, mail-back, one-day trip diary), a marketing/behavior change intervention (dialogue marketing technique with market segmentation), and an after survey. Each phase lasts approximately six weeks. The FTA's pilot project was based on personalized, individual marketing of potential commuters who may have considered using public transit, but needed more information. Transit agencies in the pilot communities first identified a neighborhood (approximately 600 households) with existing transit service, and those residents were contacted in writing to determine if they were interested in learning more about travel options. Interested residents were then contacted by phone to determine if they would like information on transit, bicycling or walking. 
The outreach continued until residents had enough information to ensure their comfort level with trying different modes of transportation. In a few cases, bus operators made home visits to personally discuss public transportation routes and options with residents. Cities involved included Durham, NC; Sacramento, CA; Cleveland, $\mathrm{OH}$; and Bellingham, WA (10), (11), (12), and (13). The IMPD results measured in these cities are compiled from the referenced reports in the Table 1.

Table 1 - Summary Results of I MPD

\begin{tabular}{|c|c|c|c|c|}
\hline & Durham & Sacramento & Cleveland & Bellingham \\
\hline \multicolumn{5}{|c|}{ Sampling Statistics for the Before Survey } \\
\hline Number of surveys mailed & 2,400 & 2,600 & 2,700 & 4,400 \\
\hline $\begin{array}{l}\text { Mail returned by post office } \\
\text { (addressees not reached) }\end{array}$ & 470 & 380 & 265 & 739 \\
\hline Sample size & 1,930 & 2,200 & 2,435 & 3,661 \\
\hline Surveys completed and returned & 1,043 & 1,288 & 1,583 & 2,196 \\
\hline Response rate & $54 \%$ & $58 \%$ & $65 \%$ & $60 \%$ \\
\hline Interested & $456(51 \%)$ & $352(39 \%)$ & $478(40 \%)$ & $331(37 \%)$ \\
\hline Regular (Already using EFM) & $80(9 \%)$ & $110(12 \%)$ & $232(19 \%)$ & $289(32 \%)$ \\
\hline Not Interested & $364(40 \%)$ & $438(49 \%)$ & $490(41 \%)$ & $280(31 \%)$ \\
\hline \multicolumn{5}{|c|}{ Individualized Marketing Intervention } \\
\hline Tote bags sent with information & 268 & 220 & $\mathrm{~N} / \mathrm{A}$ & $\mathrm{N} / \mathrm{A}$ \\
\hline Home visits & 5 & 15 & 47 & 25 \\
\hline \multicolumn{5}{|c|}{ Sampling Statistics for the After Survey } \\
\hline Number of surveys mailed & 2,150 & 2,500 & 2,900 & 2,000 \\
\hline $\begin{array}{l}\text { Mail returned by post office } \\
\text { (addressees not reached) }\end{array}$ & 364 & 257 & 271 & 249 \\
\hline Sample size & 1,786 & 2,243 & 2,629 & 2,151 \\
\hline Surveys returned & 1,174 & 1,524 & 1,814 & 1,519 \\
\hline Response rate & $66 \%$ & $68 \%$ & $69 \%$ & $71 \%$ \\
\hline Control group & 593 & 780 & 920 & 659 \\
\hline Respondents in the target area & 581 & 744 & 894 & 869 \\
\hline \multicolumn{5}{|c|}{ Data Analysis } \\
\hline $\begin{array}{l}\text { Reduction in trips per person (driver) } \\
\text { per year }\end{array}$ & $7 \%$ & $2 \%$ & $4 \%$ & $8 \%$ \\
\hline $\begin{array}{l}\text { Increase in trips per person (driver) } \\
\text { per year }\end{array}$ & $7 \%$ & $1 \%$ & $5 \%$ & $10 \%$ \\
\hline \multicolumn{5}{|c|}{ Increase in Environmentally Friendly Modes of Travel } \\
\hline $\begin{array}{l}\text { Car (driver) trips replaced by EFM } \\
\text { modes }\end{array}$ & $15 \%$ & $15 \%$ & $13 \%$ & $35 \%$ \\
\hline Biking increase & $25 \%$ & $30 \%$ & $33 \%$ & $13 \%$ \\
\hline Increase in public transit use & $35 \%$ & $43 \%$ & $26 \%$ & $14 \%$ \\
\hline \multicolumn{5}{|c|}{ Everyday Mobility } \\
\hline Target group & $\begin{array}{l}900 \text { persons } \\
(770 \text { cars })\end{array}$ & $\begin{array}{l}900 \text { persons } \\
(780 \text { cars })\end{array}$ & $\begin{array}{c}1,200 \text { persons } \\
(1,040 \text { cars })\end{array}$ & $\begin{array}{l}900 \text { persons } \\
(690 \text { cars })\end{array}$ \\
\hline Reduction in VMT reduced per year & 530,000 & 160,000 & 430,000 & 250,000 \\
\hline Relative reduction in VMT & $11 \%$ & $4 \%$ & $8 \%$ & $8 \%$ \\
\hline
\end{tabular}




\subsubsection{TravelSmart Australia}

TravelSmart implements Travel Blending $₫$, a technique, used in Australia, Europe, and South America, for encouraging people to make more efficient and environmentally sound transportation choices. The technique involves governments encouraging citizens to deal with a greater number of tasks on one given trip rather than making several trips to multiple locations at different times, thus improving travel efficiency and decreasing the strain on transportation networks. In Adelaide, Australia, a public awareness campaign called Travel Blending $\AA$, introduced ways for individuals to reduce car use by blending, or mixing, their travel choices over time (14). The program involved the development of a unique travel awareness campaign while working directly with households to:

- Measure current travel behavior using a travel diary instrument

- Provide personalized information for making simple, incremental changes to travel behavior using a feedback sheet

- Give people a chance to implement the suggested incremental changes

- Measure travel behavior changes using a second travel diary

In all, 84 percent of participating households in the Adelaide trial received some feedback. Nearly three quarters of the participating households (72\%) received comparative feedback for at least some members of the household. This comparative feedback provided information about changes they had made during the program. Although the diaries are primarily a tool for participants to observe their own behavior, one of the benefits of the two-diary format for the Travel Blending ${ }^{\circledR}$ program is the ability to measure changes in reported travel behavior and car use. Results from Adelaide indicate about a 10 percent reduction in vehicle kilometers traveled and a slightly higher percentage reduction in vehicle trips and total hours spent in the car. These results, while encouraging, must be interpreted cautiously. Further research was recommended to explore the generality and magnitude of travel behavior changes due to travel blending.

\subsubsection{U.K.'s Personalized Travel Planning}

Personalized travel planning (PTP) is a generic term covering a range of targeted marketing techniques aimed at encouraging people to switch some of their car trips to walking, cycling or public transport (15). In December 2002, the U.K. Department for Transport (DfT) awarded grants to 14 local authorities in England to run pilot personalized travel planning projects. Of these, seven targeted residential populations, six targeted workplace populations, and two targeted schools (one Local Authority covered two types of target population).

The pilots that targeted residential populations were consistently the most effective at reducing car kilometers and increasing use of sustainable modes of 
transport. All seven residential pilots saw a modal shift away from car use, with estimated reductions in car use over a year ranging between 0.05 million and 6.2 million car kms.

The workplace pilots tested a wider range of approaches and methodologies with wider variations in results than for the residential pilots. The monitoring and evaluation analysis provided in some reports was limited. With the exception of two employers that did not record a reduction, the workplace pilots demonstrated a reduction in car kilometers of between 18,000 and 186,000 car kilometers per year.

The two school pilots reported a modal shift away from car use, but control groups were not used in these projects, and therefore it is not possible to establish whether this modal shift was due to the personalized travel planning intervention, or because of external factors affecting modal choice. The monitoring and evaluation analysis provided for the school pilots did not include the distances traveled, and therefore it was not possible to estimate the total number of car kilometers saved as a result of the school based personalized travel planning pilots, and neither was it possible to calculate the cost per km saved.

\subsubsection{Japan's Travel Feedback Program}

In Japan, researchers conducted a field experiment to investigate the effectiveness of a travel feedback program for reducing family car use (16). A total of 292 fifth-grade students (10 and 11 years of age) and members of their families participated in the experiment. Over 150 participants received individualized information and advice on reducing family car use (the advice group). About 140 participants were asked to make behavioral plans with respect to methods of reducing car use (the planning group).

Six weeks prior to experimental intervention, the students and their families answered a questionnaire on the frequency of car and public transport use over three consecutive days (Sunday, Monday, and Tuesday), and the number of days the family car was used each month. Four weeks before the experiment, all students were lectured on the impacts of global warming, the role that $\mathrm{CO}_{2}$ plays, and $\mathrm{CO}_{2}$ emission levels from car use. One week later, participants in both groups answered an exact duplicate of the original questionnaire. It was found that families encouraged to make behavioral plans with respect to methods to reduce car use actually made such reductions. The actual reduction was estimated to be 27.7 percent in terms of total trip duration, and 11.6 percent in terms of car-use days. By contrast, households that merely received advice on how to reduce car use did not make similar changes. Theories of implementation intention state that forming an intention to implement a behavioral plan is necessary in the actual implementation of that behavior. Encouraging individuals to make behavioral plans 
provides a more powerful incentive by directly urging them to form implementation intentions.

The research team drew upon the experiences from the aforementioned efforts in developing the Personal Travel Coach. For example, the use of a diagram in the Japanese field experiment's feedback led to the inclusion of a map showing each trip. The families in the Japanese advice group were asked to fill in a three-day activity-travel diary on starting times, ending times, types and locations for all their activities, and mode of transportation used in traveling to and from activities. Upon analyzing the diaries, each family was presented with a diagram of their three-day activity-travel patterns and how the patterns might be modified to reduce $\mathrm{CO}_{2}$ emissions. Each household in the planning group was asked to develop behavioral plans to modify up to three of their home-based car trip chains with the aim of reducing $\mathrm{CO}_{2}$ emissions. They were asked to log their planned departure time, arrival time and travel mode for each trip, and the location of each stop in their modified car trip chains.

\subsection{Types of Feedback and Delivery methods}

A sample of feedback advice to one of the users for one trip occurrence from TRAC-IT Phase 1 was:

- Your Trip on 3/22/2005 from WALGREENS to CIRCUIT CITY was less than 1 mile.

$\checkmark \quad$ Please consider walking or biking to this location next time.

- Your Trip on 3/22/2005 from STAPLES to Home was less than 3 miles.

$\checkmark \quad$ Please consider biking to this location next time.

- Your Trip on 3/22/2005 from Home to STAPLES was less than 3 miles.

$\checkmark \quad$ Please consider biking to this location next time.

Examples of other advice proved the Expert System component of the Personal Travel Coach technically works, but logically needed to improve the recognition of certain combinations of trip purposes. Suggestions for modifying trips need to be refined from the TRAC-IT version developed under Phase 1. For example:

- TRAC-IT in Phase 1 advised people to walk to a nearby gas station.

- Your Trip on 3/25/2005 from Home to gas station was less than 1 mile.

$\checkmark \quad$ Please consider walking or biking to this location next time.

- TRAC-IT in Phase 1 advised people to essentially drop their child off at daycare on Monday and pick them up on Friday.

- You took 2 trips within one week of 03/23/2005 to child's school.

$\checkmark \quad$ Please consider combining these trips into a single trip. 
- You took 2 trips within one week of 03/24/2005 to day care.

$\checkmark \quad$ Please consider combining these trips into a single trip.

The feedback system worked based on the rules input into the system; however, the Phase 1 report concluded that these rules needed to be more descriptive and less general which is one of the objectives of this research project.

TravelSmart sends a general statement, "Everyone: Remember when you share a ride with someone instead of driving yourself, this is a real benefit to the environment in Adelaide. On the other hand, when someone makes a car-driven trip especially to take you somewhere that you could walk, ride, or even take a bus or train to, traveling as a car passenger does not help to reduce congestion and pollution."

The following list includes examples illustrating the type of feedback suggestions made to participants:

- Craig, would it be possible for you to travel by public transport one day a week or one day a fortnight? You could catch the train from Blaxland Station and change to the 301 bus at Central Station. We have enclosed copies of the train times that seem to suit your travel pattern.

- Julie, we noticed that there were never any occasions on which you did two or more things on one car journey. This is often called trip chaining, and many people use it to reduce their car trips.

- Graham, when you have the choice of using the Commodore or the Statesman, try to use the Commodore (if permitted) because it is less polluting.

The marketing package developed for the ODOT IndiMark Pilot Project consisted of a range of alternative transportation informational materials and incentive items, as well as the offer of face-to-face advice for households on walking, bicycling, and/or public transportation modes of transportation. Alternative transportation materials and services were listed on Service Sheet order forms that were mailed to households during the Service Phase of the IndiMark process. Project participants were then able to select which items on the Service Sheet they were interested in receiving. After Socialdata America received each respondent's Service Sheet, a personalized package (tote bag) was assembled and delivered by bicycle to each household. Individual households identified in the Contact Phase as Interested, received a package of informational materials and other transportation-related services that were tailor-made to their individual needs. Experience from other IndiMark projects around the world shows that this leads to greater satisfaction and use of the information and services provided. 


\subsection{Evaluating Travel Behavior Changes}

For the IMDP, the main criterion used for evaluating the demonstration was the reduction in total vehicle miles traveled per year (341 days) calculated from before and after surveys. Table 2, generated from several resources listed at the end of this document, summarizes performance measures used for IndiMark and Travel Blending. The reported criteria were decrease in vehicle kilometers, reduction in drive-alone trips, and increase in public transit trips.

A performance measure common among these studies is the reported reduction in vehicle miles of travel. The potential advantage of using an electronic method to collect travel survey data should be the higher accuracy in reporting reduction in vehicle miles traveled before and after tailored feedback advice is provided. The estimated reduction in emissions and fuel consumption can be calculated electronically based on the type of vehicle used, the time of day the trip was taken, and the distance traveled, all of which are readily gathered via the TRAC-IT prototype. Other performance measures include reduction in single-occupancyvehicle (SOV) trips and increased use of public transportation and non-motorized modes of travel. It would also be interesting to note before and after perceptions of participants when informed of the amount and cost of SOV traveling they undertook and how minor adjustments in travel patterns, such as moving their trip time out of the peak hour and/or trip chaining, may save them time and money.

\subsection{Summary}

As evident by the literature reviewed and presented in this report, travel behavior can be impacted by individualized marketing techniques that encourage household members to alter their travel decision-making process particularly after looking at a snapshot of their daily trips. Evaluations of large-scale projects using IndiMark have shown an average relative reduction in car kilometers traveled by up to 19 percent in Germany, 17 percent in Australia, and 13 percent in Sweden. Total VMT reduction per year ranged from 0.04 to 13.00 percent in American cities implementing IMDP (Table 2). In Japan, the field experiment yielded estimates of 27.7 percent reduction in total trip duration and 11.6 percent reduction in car-use days. It is impractical to compare these case studies to get an understanding of what travel blending techniques work best and why. Each seems to work in its own environment to varying degrees. Many factors play into the success of what is referred to in the literature mostly as pilot or demonstration projects, implying that the research community is still examining the how and where of these techniques. Ultimately, the goal is to implement programs that ensure the designed outcome is worth the investment. Variation in level of effort and time invested in the outreach can change the outcome. For example, FTA's IMDP's outreach continued until residents had enough information to ensure their comfort level with trying different modes of transportation, while TravelSmart allows households four weeks to practice changing their travel patterns after personalized feedback is 
provided before an evaluation is conducted. Methods of assessing the specifics of household socio-economics, cultural characteristics, and environmental awareness are an important factor. For example, the families in the advice group in the Japanese experiment were asked to fill in a three-day activity-travel diary on starting times, ending times, types and locations for all their activities, and mode of transportation used in traveling to and from activities, while the IMDP used a self-administered, mail-back, one-day trip diary. Consequently, the tailored travel feedback will depend on the methods of survey and analysis. Also, these pilots had different emphases within the outreach phase. The Australian pilot was promoting trip chaining as a means of reducing car use, while the IMDP promoted transit, bicycling, and walking.

Table 2 - Selected I ndiMark and Travel Blending Evaluation Criteria

\begin{tabular}{|c|c|c|c|}
\hline $\begin{array}{l}\text { Approaches to targeted } \\
\text { marketing }\end{array}$ & $\begin{array}{l}\text { IndiMark in South } \\
\text { Perth }\end{array}$ & $\begin{array}{c}\text { IndiMark in district of } \\
\text { Brisbane }\end{array}$ & $\begin{array}{c}\text { Travel } \\
\text { Blending in } \\
\text { Adelaide }\end{array}$ \\
\hline Household participation & $\begin{array}{l}400 \text { approached, of } \\
\text { which } 36 \% \\
\text { expressed an interest } \\
\text { in switching from car } \\
\text { to other modes and } \\
\text { were sent } \\
\text { information on } \\
\text { alternative modes }\end{array}$ & $\begin{array}{l}\text { Information sent to } 196 \\
\text { promising households out } \\
\text { of the } 455 \text { invited to } \\
\text { participate (households } \\
\text { that had no interest in } \\
\text { participating or already } \\
\text { used alternative modes } \\
\text { were excluded) }\end{array}$ & $\begin{array}{c}70 \% \\
\text { penetration } \\
\text { rate with } 900 \\
\text { participating } \\
\text { households }\end{array}$ \\
\hline $\begin{array}{l}\text { Reported vehicle kilometers } \\
\text { reduced }\end{array}$ & $14 \%$ & NA & $11 \%$ \\
\hline $\begin{array}{l}\text { Reported reduction in drive- } \\
\text { alone }\end{array}$ & $10 \%$ & $10 \%$ & NA \\
\hline $\begin{array}{l}\text { Increase in public transit } \\
\text { trips }\end{array}$ & $21 \%$ & $33 \%$ & NA \\
\hline $\begin{array}{l}\text { Estimated reduction in } \\
\text { greenhouse gas emissions }\end{array}$ & $\sim 14 \%$ & NA & $\sim 11 \%$ \\
\hline $\begin{array}{l}\text { Estimated pollutant } \\
\text { reduction }\end{array}$ & $\sim 14 \%$ & NA & $\sim 11 \%$ \\
\hline \multirow[t]{3}{*}{ Other reported impacts } & $\begin{array}{l}91 \% \text { increase in } \\
\text { cycling trips }\end{array}$ & $\begin{array}{l}6 \% \text { increase in cycling } \\
\text { trips }\end{array}$ & NA \\
\hline & $\begin{array}{c}16 \% \text { increase in } \\
\text { walking trips }\end{array}$ & NA & NA \\
\hline & $\begin{array}{l}9 \% \text { increase in trips } \\
\text { by car passengers }\end{array}$ & NA & NA \\
\hline
\end{tabular}

The lessons learned from these other travel behavior change feedback projects were considered in the design of the TRAC-IT applications including the graphical user interface and the wording of feedback suggestions. 


\section{Chapter Three - Personal Travel Coach: Expert System Module}

This chapter presents the Personal Travel Coach developed in Phase 2 of this research series. The enhanced Personal Travel Coach consists of two modules: the TRAC-IT Expert System, discussed in this chapter, and the TRAC-IT Path Prediction system, discussed in Chapter 4. The next section reviews the logical and technical progression in the development of the Expert System based on the findings of TRAC-IT Phase 1. The targeted areas of improvement are discussed, and the new version of the TRAC-IT Phase 2 Expert System is presented, along with a complete description of the rules used to generate suggestions.

\subsection{TRAC-IT Expert System Concept}

The TRAC-IT system from Phase 1 was able to successfully upload trip data from a mobile device to the server and provide suggestions for more efficient transportation options based on the household travel behavior. The Expert System was designed to test the ability of collecting and using data from TRAC-IT. The preliminary Expert System then generated tailored feedback for each user, based on his or her household travel behavior over the entire test period. A simplified illustration of sample advice delivered to a participant via text message is provided in Figure 1.

The TRAC-IT expert system is a rule-based, automated system that analyzes individual and household travel behavior data and generates personalized feedback to the user. This feedback contains specific travel advice such as carpooling or information about using alternate modes of transportation that could potentially save the user time and/or money. The TRAC-IT Expert System rules for this research project were developed by the TDM community for use in travel behavior change field studies (2). The rules are used to analyze trip data in order to identify inefficient travel behavior and construct meaningful feedback to users based on specific trips that the user recorded. 


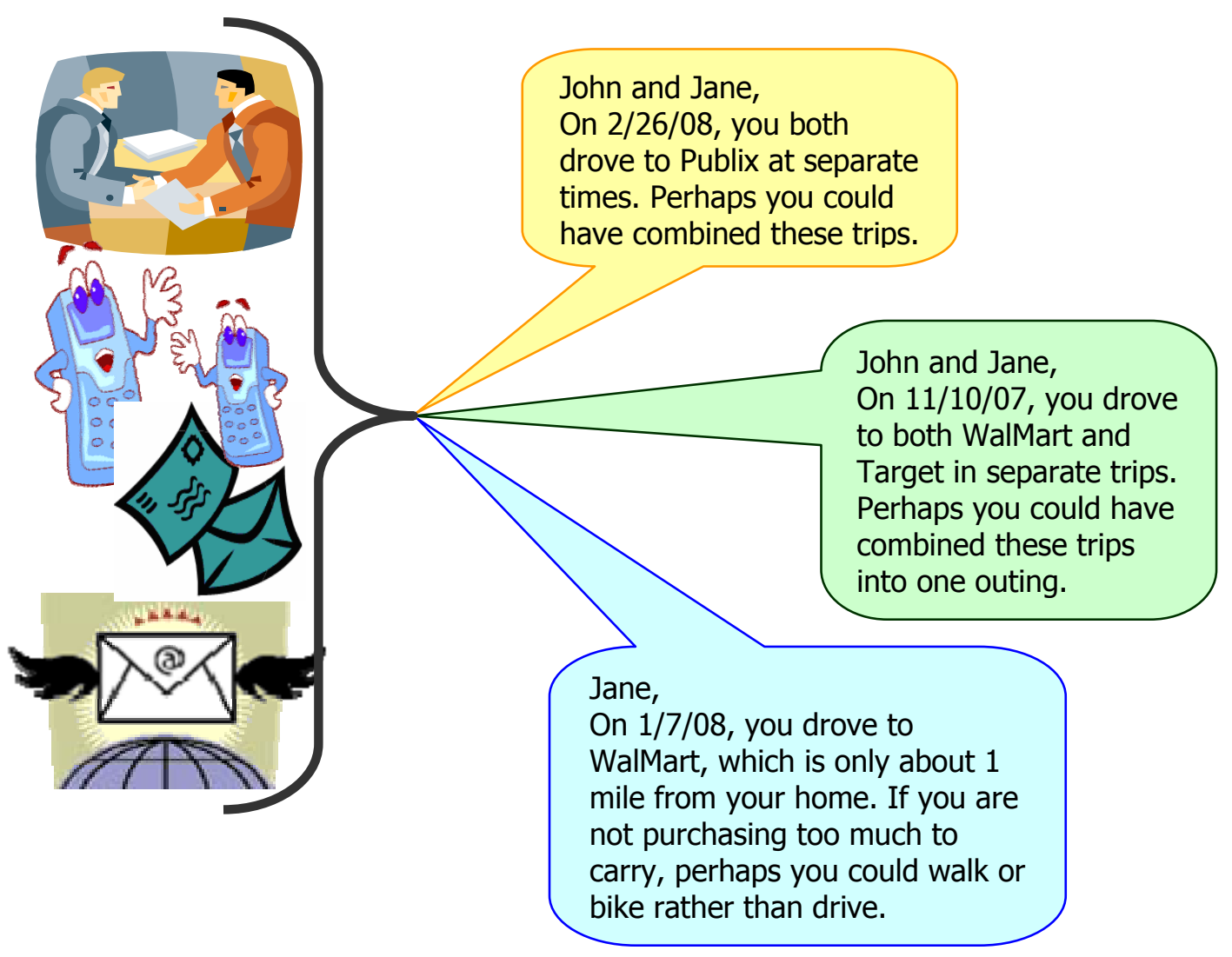

Figure 1 - Expert System Feedback: an illustration of sample text messages

The conceptual data flow in the TRAC-IT Expert System is illustrated in Figure 2. Travel behavior data are recorded using GPS-enabled mobile devices. Each trip containing GPS and user-entered survey data is then input to the Expert System and processed by a series of rules. If the trip successfully meets the conditions of a specific rule, then advice is generated. For example, if the trip was to a grocery store that is less than one mile and the participant drove, the system generates a suggestion to bike or walk. If the trip does not meet the conditions of a specific rule, then the trip is deemed efficient by the Expert System and no feedback is generated for that rule. For example, if a trip is the same trip length and the same purpose as the one before, but the user already biked or walked instead of driving, no suggestions are generated. 


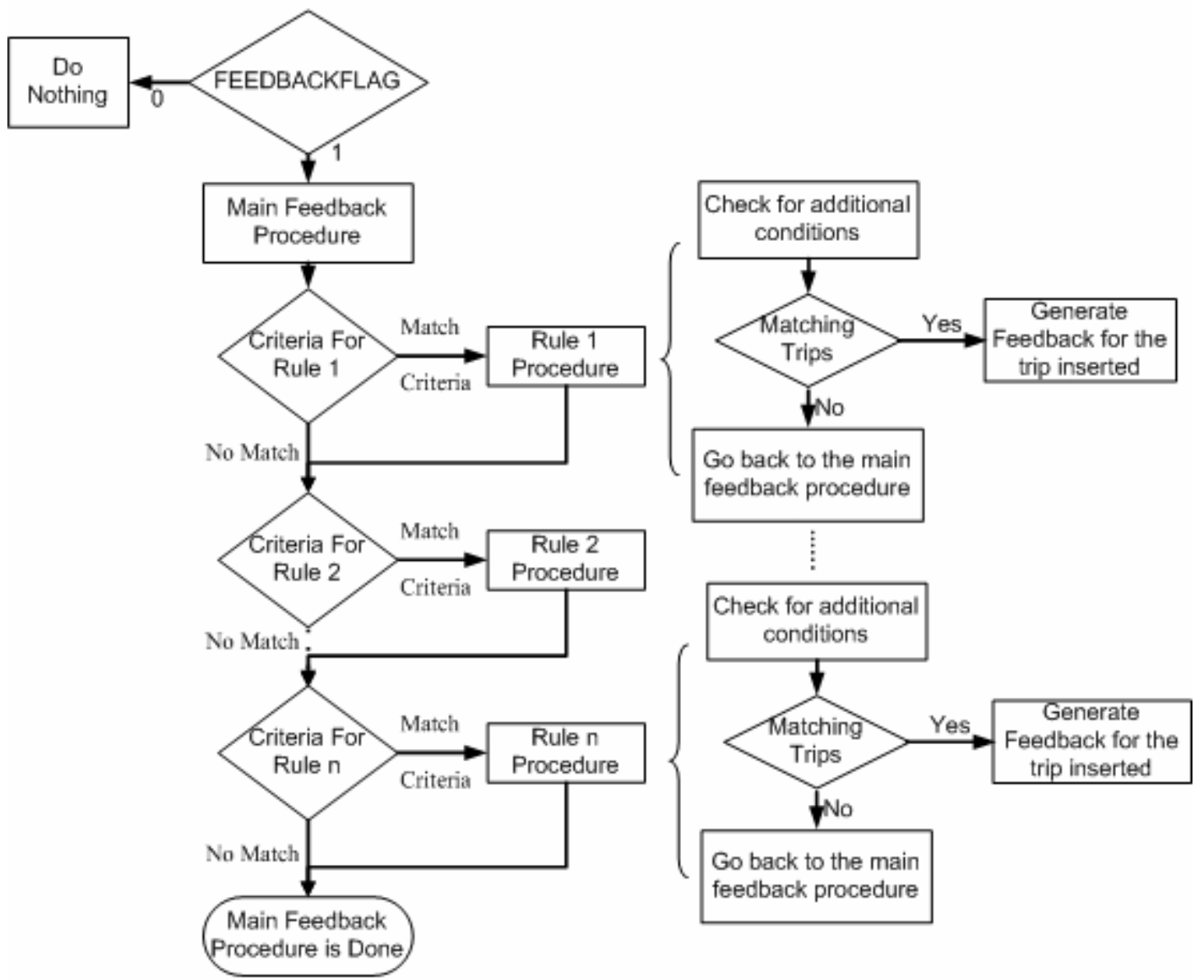

Figure 2 - Conceptual Flowchart of the TRAC-IT Expert System

\subsection{Limitations of TRAC-IT Phase 1 Expert System}

As a result of the testing performed in TRAC-IT Phase 1 it was concluded that the TRAC-IT Expert System works. However, the implementation of the original system was done in a scripting language referred to as Transact-SQL (T-SQL) (17). This particular script language (Figure 3 ) caused the system to experience functional limitations. 


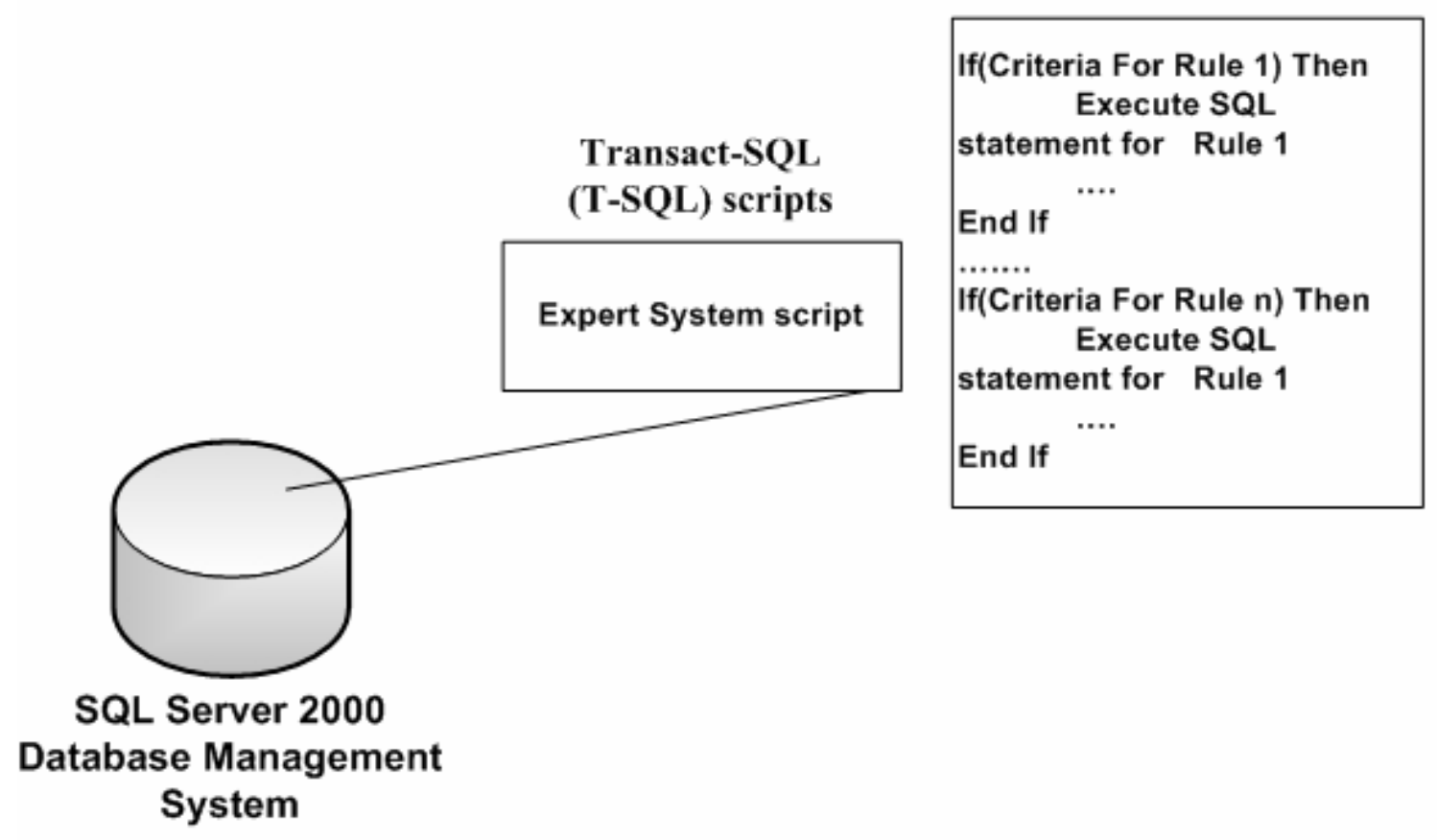

Figure 3 - Transact-SQL I mplementation Module of the TRAC-IT Phase 1 Expert System

One of the goals of Phase 2 was to overcome these challenges from the Phase 1 implementation. Nine Phase 1 limitations are briefly discussed below:

(1) Lack of portability

The T-SQL scripting language is proprietary to Microsoft SQL Server, and therefore the original Phase 1 Expert System cannot be used with another database system such as Oracle or Postgres (18) (19). This limits the functional implementation of the feedback system to the utilities available within Microsoft SQL Server, which excluded advanced Geographic Information System (GIS) functionality such as spatial queries not supported by SQL Server (20). Spatial queries are important for advanced TRAC-IT functionality such as the prototype Path Prediction system discussed in the next chapter.

(2) Mixed error checking, preconditions, and rule logic

The TRAC-IT Phase 1 Expert System was written completely in the T-SQL scripting language that mixes error checking, preconditions, SQL statements to retrieve data, and rule logic. That made it very difficult to change one script item without changing others, then having to reset and retest the entire system.

(3) Implementation diverged from conceptual flowchart 
As previously mentioned, Figure 2 represents the logical data flow of the Expert System execution process that separates components into pieces based on functionality. However, since T-SQL is a scripting language that does not support object-oriented design, the implementation of the Phase 1 system, seen in Figure 3 , did not directly mirror this design. Instead, multiple components were necessarily blended together since distinct separation of modules was not supported. This feature made changes to the Phase 1 system difficult since the logical data flow must be continuously re-mapped to existing implementation in the script.

\section{(4) Lacked object-oriented concepts}

Three definitions used to describe object-oriented concepts need to be made clear before discussing this limitation. Inheritance defines a set of characteristics that can be implemented once and then inherited by another set that has all properties of the first. Encapsulation describes a principle by which the characteristics of a trip or user, represented by objects in the Expert System software, can be easily enclosed into a single entity which represents the entire trip or user. Lack of encapsulation forces trip and user properties to be input to a function and processed one-by-one instead of being enclosed in a single object, which makes the future inclusion of new properties difficult without system-wide changes. Entity relationships allow the representation of relationships between rules and preconditions as objects within the software of the Expert System.

T-SQL is a simple scripting language that does not support object-oriented concepts including inheritance, encapsulation, entity relationships, and re-using modules. Additionally, since geographic objects are not supported by T-SQL, spatial analysis within the script is not possible.

\section{(5) Relationship of trips to users}

Since object-oriented programs are not supported in T-SQL, there is no direct representation of relationships in data that is being processed inside the script or in the rules themselves. Therefore, rules that process relationships of users to trips and users to users are very difficult to implement since all relationship analysis must take place in SQL statements and not inside the script. This requirement also forces the implementation to blend error checking, preconditions, rules, and optimization since all these actions must be implemented within the SQL statements.

\section{(6) Difficult to add new rules}

Mixed functionality between script and SQL statements, including SQL optimizations coupled with the lack of object-oriented concepts made it difficult to add new rules to the Expert System. New preconditions and error checking 
procedures must be implemented and tested for each new rule since they will contain logic specific to the new rule that is mixed with old logic from past rules.

\section{(7) Difficult to maintain}

SQL Server has limiting debugging capabilities for T-SQL scripts, and therefore it is very difficult to analyze and repair issues as they arise (21). Additionally, proficiency in T-SQL is rare for application developers and, therefore, there is a large learning curve for most developers attempting to maintain the system. This can limit wide-scale deployment and support of TRAC-IT.

(8) Duplication of code for error checking and preconditions

Reuse of objects is not possible when rules, error checking, and preconditions are mixed with SQL statements and SQL optimization. Therefore, code that accomplishes similar tasks for multiple rules such as error checking and preconditions must be duplicated. Code duplication increases the cost of continued development by increasing the level of effort necessary to analyze and debug the system.

(9) Same information accessed multiple times within different rules

Since Expert System feedback logic is mixed with SQL statements that implement rules, the same data may be pulled and discarded from the database multiple times between rules, or even within the same rule. This results in an inefficient implementation that unnecessarily taxes system resources and limits scalability. 


\subsection{Design of TRAC-IT Phase 2 Expert System}

The TRAC-IT Phase 2 Expert System, shown in Figure 4, was redesigned and implemented in the C\# .NET programming language in order to overcome the limitations of the Phase 1 system.

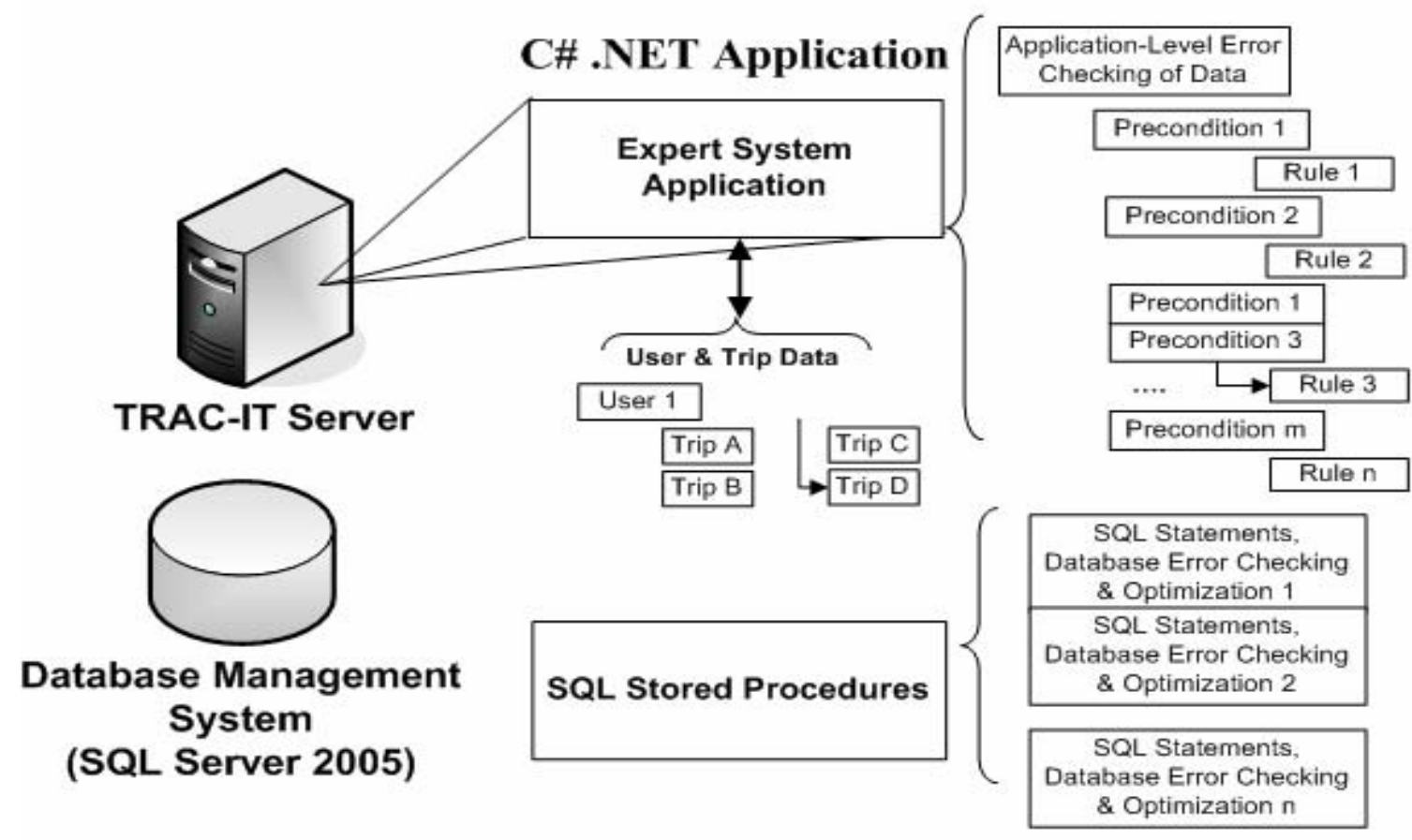

Figure 4 - Implementation of the TRAC-IT Phase 2 Expert System 
The Phase 2 Expert System features the following advantages:

(1) Portable to any database system

The main application executes independently of the database management system and can therefore be re-used on any database management system. SQL Stored Procedures, including error checking and optimization, can be created for a new database without modifying the main expert system application which contains the rule logic. The .NET Framework allows tight coupling to SQL Server 2005 if desired so the application could be triggered by database events such as a new trip being inserted into a table. An important advantage in portability is that other database management systems that directly support spatial queries on geographic data features, such as Oracle Spatial or PostGIS, can be used to further enhance the Personal Travel Coach (18) (19). The prototype of the Path Prediction module, implemented as part of the TRAC-IT Phase 2 research project, takes advantage of spatial queries to provide real-time traffic incident feedback to travelers. The Path (Route) Prediction module is discussed in detail in the next chapter.

(2) Separated and modular error checking, preconditions, and rule logic

By separating system components in an object-oriented implementation, each component can now be reused when possible. This efficient design means that each piece can be modified without impacting the functionality of other interfacing modules. This leads to easier addition of new rules as well as modification of existing rules.

(3) Implementation directly maps to logic diagram

The object-oriented support of C\# and the .NET Framework allows the implementation of the Phase 2 Expert System to directly mirror the logical diagram of its execution processes (24). This enables easier modification as conceptual additions can easily plug into the corresponding place in the actual implementation.

(4) Object-oriented concepts are enabled in data processing, preconditions, and rules

C\# and the .NET framework are fully object-oriented, allowing for the representation and processing of data in objects. Inheritance, encapsulation, entity relationships, and re-use of modules were made possible in the Phase 2 design. Also, geographic objects can be supported directly inside of the Expert System application code through Application Programming Interfaces (APIs) such as ESRI's ArcObjects. 
(5) Hierarchical representation of rules and data

Since objects are now supported in expert system execution, it is much simpler to represent conceptual relationships between data and rules. This is very important for complex rules that relate trips and users, including rules that are based on a relationship between users.

\section{(6) Ease of adding new rules}

Since rules are completely separated in the implementation from the preconditions and error checking, it is convenient to add new rules without having to test and debug the entire system. Also, existing preconditions and error checking can simply be reused without havinh to re-write and test them specifically for new rules. Multiple preconditions and error checking can also be chained together to form new types of preconditions that do not require extended debugging.

\section{(7) Easy to maintain - standard C\# .NET code from managed environment}

Visual Studio.NET is used as the development environment for C\# applications and has full support for interfaces with different types of database management systems as well as full debugging support (25). Additionally, C\# is a common language that is very close to Java in syntax, and therefore has only lesser learning curve than T-SQL for most application developers.

\section{(8) No duplication of code}

The object-oriented capabilities of the Expert System allow modules to be reused. If a new rule is added and existing preconditions and error checking modules meet the needs of this rule, those modules can be reused and there is no duplication of code to maintain and debug. Additionally, new rules can chain previous preconditions and error checking modules along with new preconditions or error checking modules, thereby supporting the use of existing building blocks to form more complex rules.

\section{(9) Trip and user data pulled from database only once per execution}

Since the expert system logic is completely separated from SQL statements that retrieve data, the data for a set of trips or users can be pulled from the database and represented as related objects in memory only once for the entire expert system analysis. This design results in reduced impact on database management system resources and promotes system efficiency.

(10) New rules

To illustrate the strength of these design concepts, a new rule to avoid rush hour was created and implemented in the system. As previously stated, some of 
TRAC-IT Phase 1 feedback fits the logical rules of the Expert System but did not make sense based on trip conditions. For example, a participant dropped off and picked up her child at daycare daily was given a suggestion, "We noticed that you made multiple trips on different days to the same destination within one week; why not combine these trips and travel to that destination once a week?" The feedback was given because the specific trip purpose of dropping children off was not exempt from the rule of combining multiple trips made on different days to the same destination. For this rule, several new preconditions were added to the TRAC-IT Phase 2 system to suppress feedback if the purpose of the trip was of specific types, including dropping off or picking up another person.

Another example from Phase 1 recommended that a participant walk to his destination of less than one mile (as walking rule would suggest) although the destination was a gas station and the driver was fueling his vehicle. To prevent this kind of feedback in the Phase 2 version, preconditions were added such that if the location type was a gas station, or if the purpose was a newly created Car Services purpose, the Expert System would not suggest walking, biking, or transit.

Additional improvements to the Expert System included modifying the format of feedback in order to make it more understandable to the user and supplying additional information such as the purpose of the trip or name of the destination within the generated feedback.

\subsection{TRAC-IT Phase 2 Expert System Module Implementation}

The TRAC-IT Phase 2 Expert System was implemented in C\# on the .NET framework using the Microsoft Visual Studio .NET 2005 environment. The system was interfaced with SQL Server 2005, which was used as the main database management system for TRAC-IT (26). The enhanced database schema, which also supports advanced GIS functionality, is shown in Figure 5.

\subsubsection{Expert System I nput}

Since the goal of the behavior change feedback system is to reduce the number of drive alone trips and/or unnecessary trips and/or vehicles miles of travel, the focus of feedback suggestions to influence travel behavior can be marketed as a benefit to participants saving time and/or money. The Expert System analyzes the trips that people make over an extended period (2-4 weeks of survey). The system processes one trip at a time through a set of rules. For example, Trip ID 777 taken by User ID 333 uses the set of rules to analyze the relationship of Trip 777 to other trips made by User 333. The rules also analyze the relationship of Trip 777 to trips taken by other household members of User 333. These analyses may offer trip chaining or carpooling within the household as well as biking, walking, or transit suggestions. Trip 777 may also be compared to trips by other users to 
generate ridesharing suggestions if the origin/destination and trip times apply. The feedback is a suggestion to the user to eliminate a trip or, if applicable, combine with other trips.

Many types of data are used as input for the Expert System, including GPS data, mode of transportation data, and trip purpose data. Figure 6 illustrates the input and output of the Expert System.

Other data in integrations into the TRAC-IT system and is used for all users. This information includes bus stop data for transit agencies. A database of Hillsborough Area Regional Transit (HART) bus stops was used for the Tampa Bay Area. 


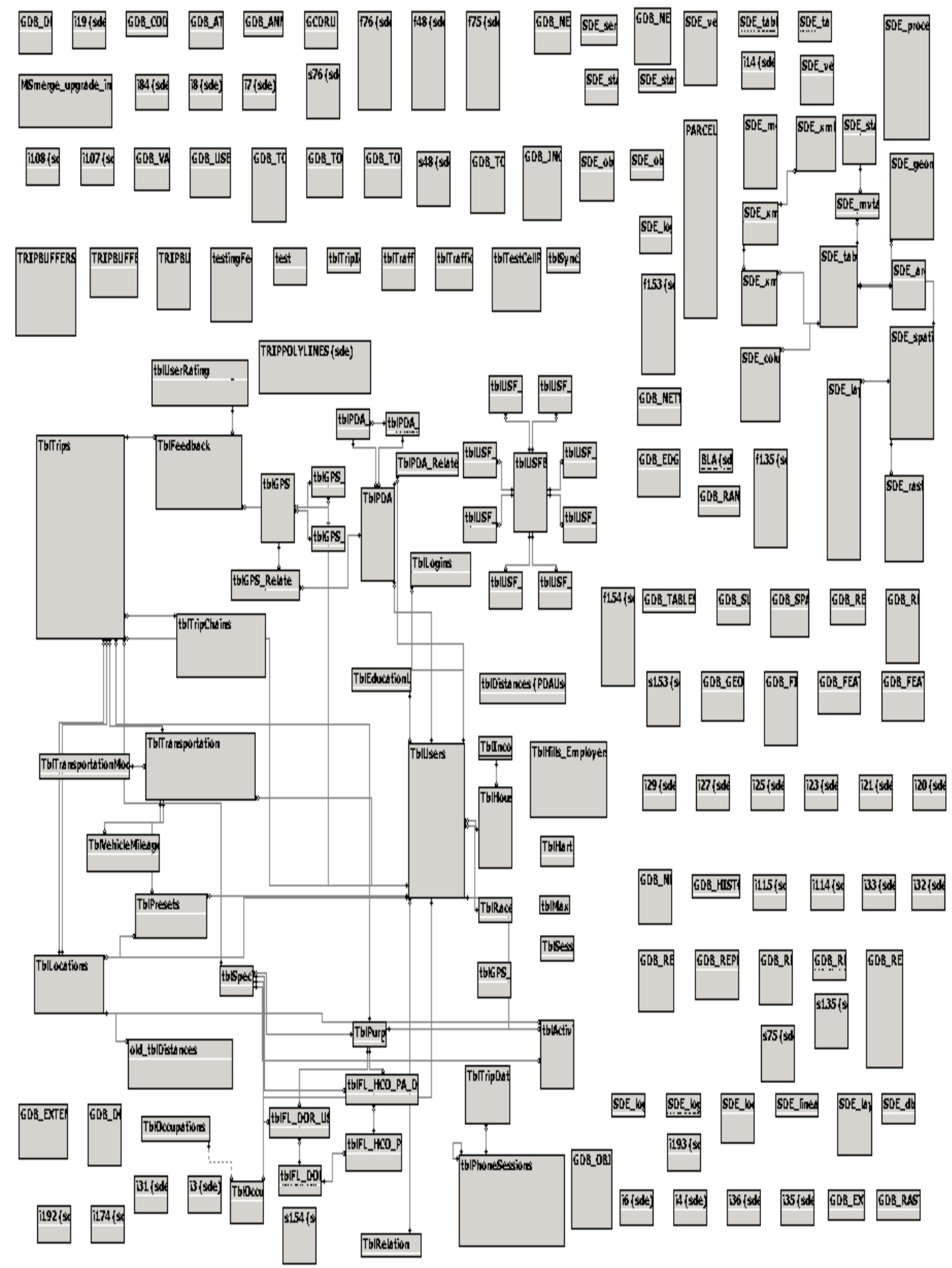

Figure 5 - Enhanced Database Schema for TRAC-IT 2 Expert System 


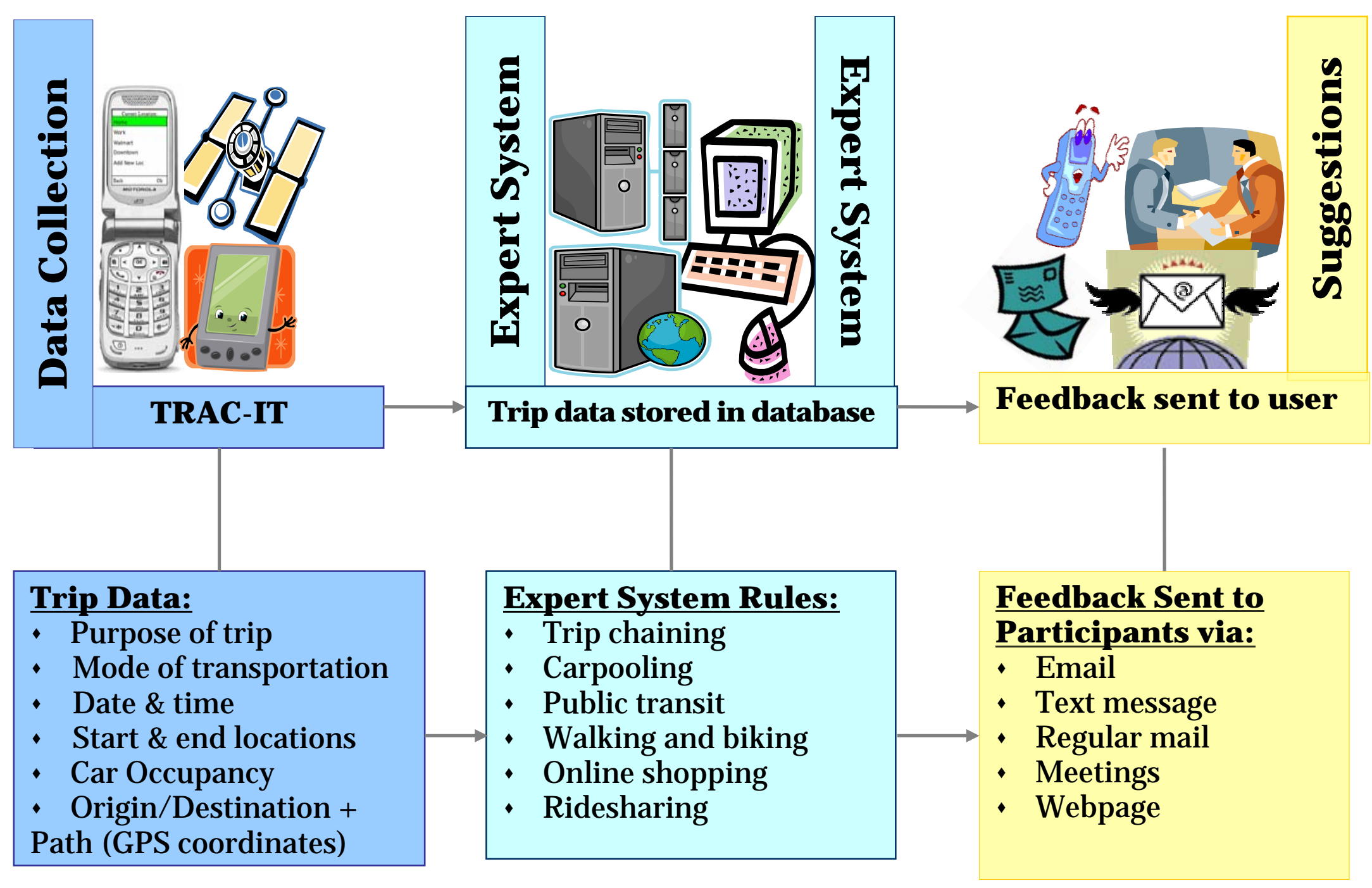

Figure 6 - Personal Travel Coach Conceptual Diagram 


\section{GPS data}

GPS data from GPS-enabled mobile phones are used to gather information such as the starting point, destination point, leaving time, arriving time, and travel path. GPS fixes, which each contain latitude and longitude information along with other data such as speed and heading, can be collected as frequently as once per second. TRAC-IT is usually set to record a GPS fix once every four seconds. Figure 7 shows a screenshot of a TRAC-IT simulation recording a trip with GPSenabled mobile phone.

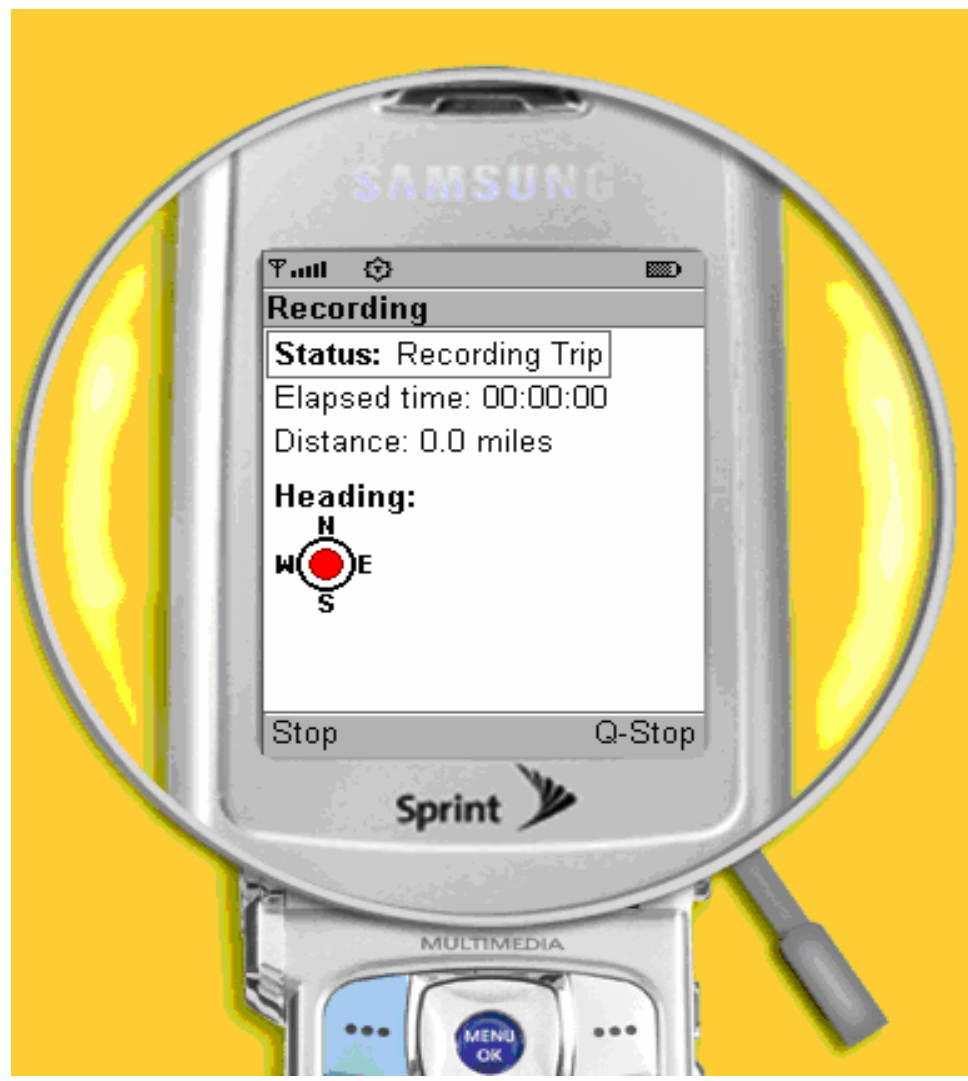

Figure 7 - Screenshot of a Simulation of TRAC-IT GPS-enabled Mobile Phone Recording a Trip

\section{Modes of Transportation}

Mode of transportation is recorded using the TRAC-IT user interface and manual user input. The modes of transportation are defined as part of the TRAC-IT system. A user may label his trip as being traveled using one of these modes of transportation, and the expert system uses this information when analyzing the trip. Figure 8 shows the menu of modes of transportation that can be selected by user. 


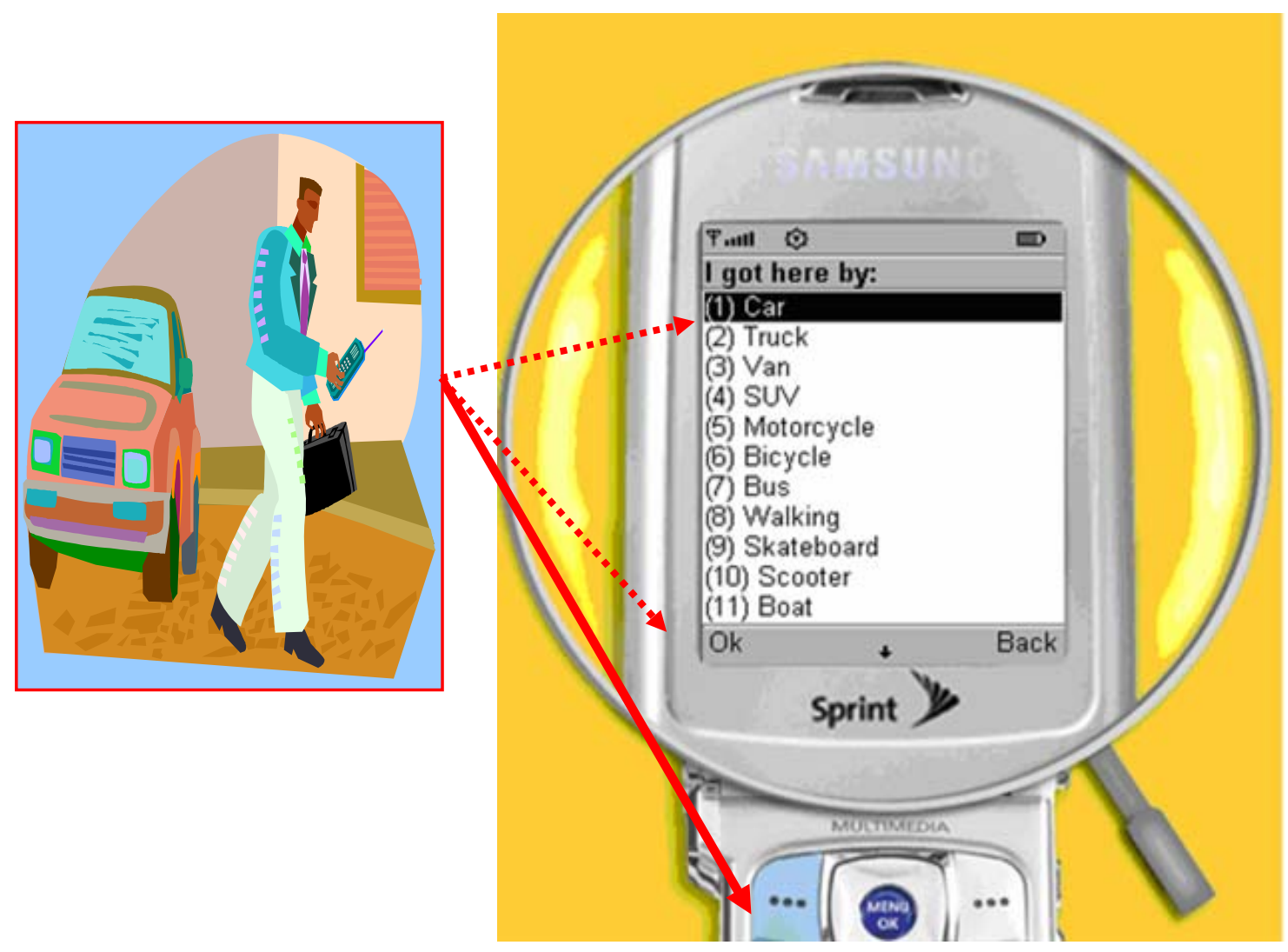

Figure 8 - TRAC-IT Menu for Modes of Transportation

\section{Trip Purpose}

The purpose and specific purpose of the trip are recorded using the TRAC-IT user interface and manual input by the user. Purposes categories and specific purposes are defined by the TRAC-IT system as shown in Table 3 . These purposes are selected by the user when they are recording their trip to indicate why they traveled to a specific location. The specific purpose is used for certain general purpose categories that require further information to narrow down possible trip purposes. The Expert System then uses these purposes and specific purposes during trip analysis. Table 3 shows the relationship between purposes and rules by illustrating which trip purposes would potentially generate feedback for specific rules. 


\begin{tabular}{|c|c|c|c|c|c|c|c|c|c|c|c|}
\hline PURPOSES & SPECIFIC PURPOSES & 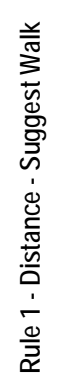 & 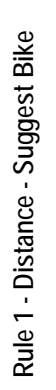 & 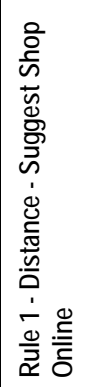 & 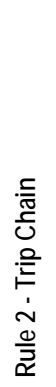 & 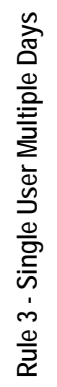 & 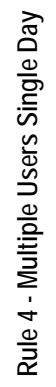 & 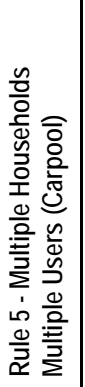 & 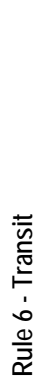 & 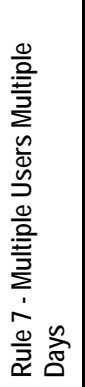 & 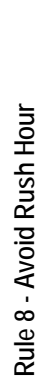 \\
\hline 1) Work & & $x$ & $x$ & & $\mathrm{x}$ & & $x$ & $x$ & $\mathrm{x}$ & & \\
\hline \multicolumn{12}{|c|}{ 2) School or Religious } \\
\hline & Go to School & $x$ & $x$ & & $x$ & & & & & & $x$ \\
\hline & Go to Religious Activity & $x$ & $x$ & & $x$ & & & & & & $x$ \\
\hline & $\begin{array}{l}\text { Go to Library: School } \\
\text { Related }\end{array}$ & $\mathrm{x}$ & $x$ & & $\mathrm{x}$ & & & & & & $x$ \\
\hline \multicolumn{12}{|c|}{ 3) Medical or Dental } \\
\hline \multicolumn{12}{|c|}{ 4) Shopping and Errands } \\
\hline & Buy Goods & & & $\mathrm{x}$ & $\mathrm{x}$ & $\mathrm{x}$ & $\mathrm{x}$ & & & $\mathrm{x}$ & $\mathrm{x}$ \\
\hline & Buy Services & $x$ & $x$ & $x$ & $x$ & $x$ & $x$ & & & $x$ & $\mathrm{x}$ \\
\hline & Car Services & & & & $\mathrm{x}$ & & & & & & $\mathrm{x}$ \\
\hline & $\begin{array}{l}\begin{array}{l}\text { Personal or family } \\
\text { business }\end{array} \\
\end{array}$ & $\mathrm{x}$ & $x$ & & $\mathrm{x}$ & & & & & & $\mathrm{x}$ \\
\hline & $\begin{array}{l}\text { Pick up or drop off an } \\
\text { item }\end{array}$ & $x$ & $x$ & & $x$ & & $x$ & & & & $x$ \\
\hline \multicolumn{12}{|c|}{ 5) Social and Recreational } \\
\hline & Go to gym, exercise & $\mathrm{x}$ & $\mathrm{x}$ & & $\mathrm{x}$ & & $\mathrm{x}$ & & & & $\mathrm{x}$ \\
\hline & Rest, vacation & $\mathrm{x}$ & $x$ & & $x$ & & $\mathrm{x}$ & & & & $x$ \\
\hline & Visit friends/family & $\mathrm{x}$ & $\mathrm{x}$ & & $\mathrm{x}$ & & $\mathrm{x}$ & & & & $\mathrm{x}$ \\
\hline & Go out: entertainment & $\mathrm{x}$ & & & $\mathrm{x}$ & & $\mathrm{x}$ & & & & $\mathrm{x}$ \\
\hline & Visit public place & $x$ & $\mathrm{x}$ & & $\mathrm{x}$ & & $\mathrm{x}$ & & & & $\mathrm{x}$ \\
\hline \multicolumn{12}{|l|}{ 6) Transportation } \\
\hline & Pickup someone & & & & $\mathrm{x}$ & & $\mathrm{x}$ & & & & $\mathrm{x}$ \\
\hline & Take and wait & & & & $x$ & & & & & & $\mathrm{x}$ \\
\hline & Drop someone off & & & & $\mathrm{x}$ & & $\mathrm{x}$ & & & & $\mathrm{x}$ \\
\hline & Change mode & & & & $x$ & & $\mathrm{x}$ & & & & $\mathrm{x}$ \\
\hline \multicolumn{12}{|l|}{ 7) Meals } \\
\hline & Eat Out & $\mathrm{x}$ & $x$ & & $\mathrm{x}$ & & $x$ & & & & $\mathrm{x}$ \\
\hline & To Go & $\mathrm{x}$ & $\mathrm{x}$ & & $\mathrm{x}$ & & $\mathrm{x}$ & & & & $\mathrm{x}$ \\
\hline 8) Return Home & & & & & & & $\mathrm{x}$ & $\mathrm{x}$ & $\mathrm{x}$ & & $\mathrm{x}$ \\
\hline 9) Other & & $\mathrm{x}$ & $\mathrm{x}$ & & $\mathrm{x}$ & $\mathrm{x}$ & & & & $\mathrm{x}$ & $x$ \\
\hline
\end{tabular}




\subsubsection{Expert System Output}

The Expert System generates suggestions to the user using the input data as well as the rules encoded in the expert system. The Expert System personalizes the suggestions by including the user-entered descriptions of the starting and/or ending locations of the trip in the output. While the actual geographic starting and ending location is recorded by GPS, latitude and longitude values, as well as addresses generated from latitude and longitude, will not be recognizable to most users for locations that they visit. Therefore, the TRACIT mobile application asks the user to enter a brief description using the keypad when they are starting or ending a trip so that the user-entered location description can be presented to the user in the suggestions generated by the Expert System. Figure 9 shows the list of locations previously entered by the current user as well as a screenshot of the user naming a new location they are visiting for the first time. Descriptions of frequently-visited locations can also be given to TRAC-IT system administrators to be entered into the system before the TRAC-IT user participates in the study in order to reduce the burden of text entry on the user for common destinations. These locations that are pre-entered into the system at the TRAC-IT server will automatically appear in the user's location list on the mobile phone.

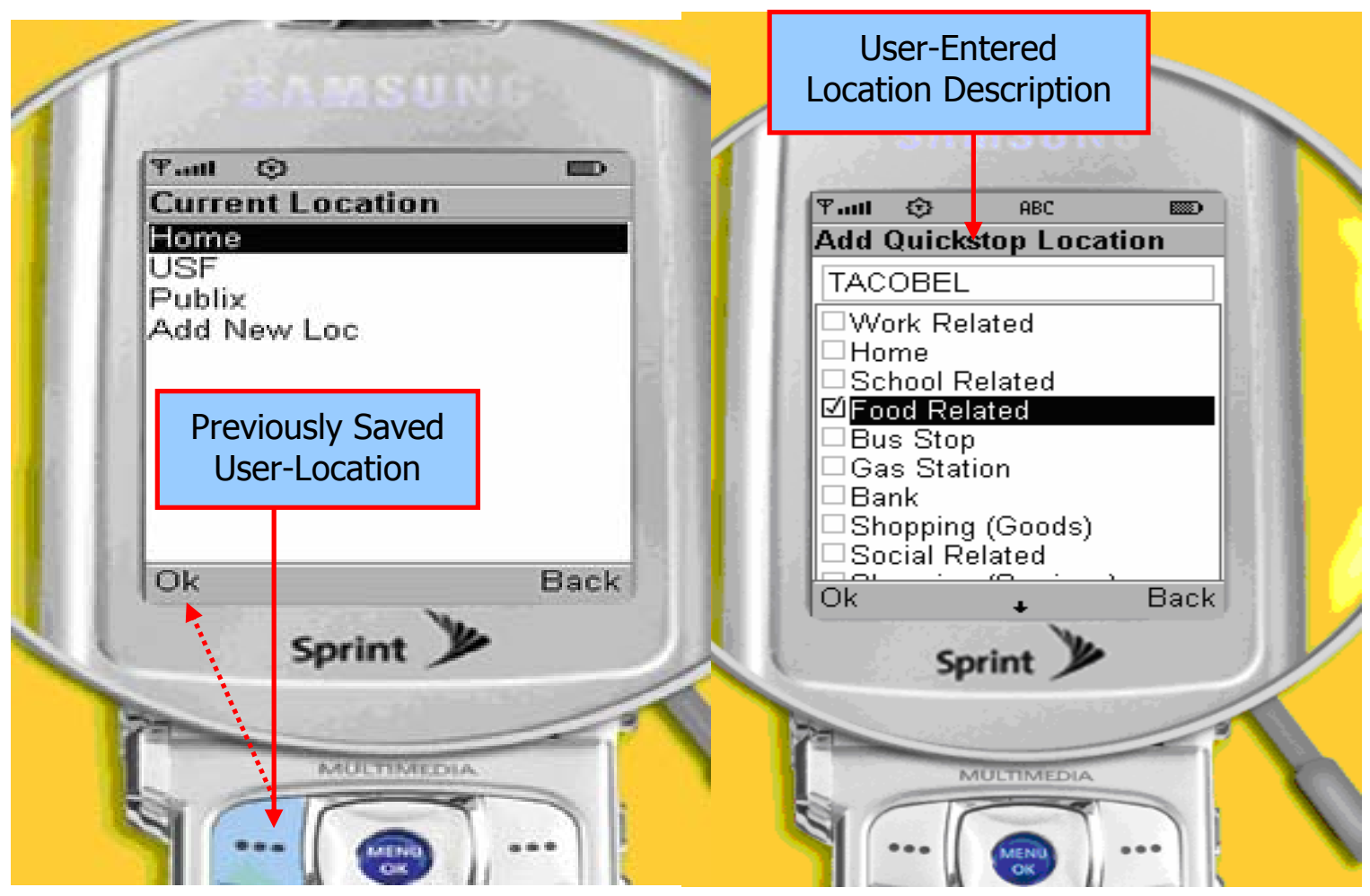

Figure 9 - TRAC-IT Mobile Application allows User-entered Location Descriptions to Assist in Personalizing Expert System Feedback 


\subsubsection{Expert System Rules}

This section highlights the purpose of each Expert System rule and gives a pseudo-code representation of the preconditions of each rule. Distance calculations are performed in miles in the TRAC-IT Expert System. Finally, it describes suggestions offered based on different patterns of detected travel behavior.

\section{Rule 1: Distance}

- ANALYZES: SINGLE USER, SINGLE TRIP

- Measures distance for a particular trip and recommends alternate modes of transportation based on distance traveled.

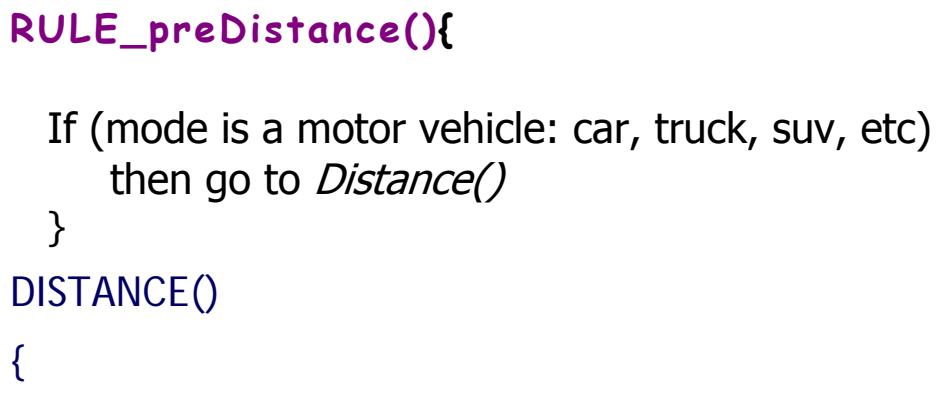

If (total dist. traveled $<=1$ AND the purposes are valid for walking) then suggest to Walk

If (total dist. traveled $>1$ AND TotalDistance $<=3$ AND the purposes are valid for biking)

\section{then suggest to Bike}

If (total dist. traveled >10 AND the purposes are valid for shopping online)

\section{then suggest to Shop Online}

\}

Rule 2: Trip Chain

- ANALYZES: SINGLE USER, MULTIPLE TRIPS, SINGLE DAY

- Determine when trip chaining should be recommended by taking the count of unique trips and the count of total trips from a starting location. If the unique trip count is less than the total trip count, then the person taking the trip on that day left the same starting location more than once in a day, so they could have stopped at one location on their way to 
another. Therefore, the recommendation to chain trips together is suggested to the individual.

- Unique Trips: number of unique trips starting at $X$ going to different places.

- Total Trips: number of trips starting at $X$ going to anywhere (can be the same place).

- If Unique Trips < Total Trips then the person taking the trip on that day left the same starting location more than once in a day, so they could have stopped at one location on their way to another.

\section{RULE_preTripChain()\{}

If (there are no past trip chaining results for that day for current user) then

UniqueTrips = number of unique trips made by current user for current day

TotalTrips $=$ number of total trips made by current user for current day

Go to Trip_Chain_Rule()

\{

TRIP_CHAIN_RULE()

ExtraTrips $=$ TotalTrips - UniqueTrips

If(UniqueTrips < TotalTrips)

then tell user how many extra trips they took that day and suggest trip chaining.

\}

\section{Rule 3: Single User Multiple Days}

- ANALYZES: SINGLE USER, MULTIPLE TRIPS, MULTIPLE DAYS

- Purpose: Determines whether a user has visited the same location multiple times during the period of 1 week previous to the day of the trip being analyzed. If so, suggest that the user should combine these trips.

\section{RULE_presingleUserMultipleDays()\{}

Count $A=$ Count the number of trips to the EndLocation that were taken during the same day by the same user that received a feedback of COMBINE_SU_MD. 
CountB $=$ Count the number of trips to EndLocation by the same user in the past 7 days that were for the purposes of Shopping or Other and the special purposes of Goods or Services.

If(CountA is 0 AND CountB $>0)\{$

\} then go to Single_User_Multiple_Days() rule

\section{SINGLE_USER_MULTIPLE_DAYS() \{}

CountB represents the number of independent trips that were taken within a week that could have been combined into a single trip.

If(purposes are valid for combining trips in one day) then CountB $=$ CountB +1

Suggest to user to combine trips (feedback type is COMBI NE_SU_MD)

\}

\section{Rule 4: Multiple Users Single Day}

- ANALYZES: MULTIPLE USERS IN SAME HOUSEHOLD, MULTIPLE TRIPS, SAME DAY

- Purpose: Determines if multiple users in a single household have visited the same locations on a single day, and if so, suggests that they travel to these locations together.

RULE_preMultipleUsersSingleDay()\{

If(mode is by motor vehicle AND purposes are valid for ridesharing AND destination is not work related AND destination is not home)

\} then go to Multiple_Users_Single_Day() rule

\section{MULTIPLE_USERS_SINGLE_DAY() \{}

Retrieve all of the trips made by other users that were taken on the same day.

For each trip, $\mathrm{X}$

Trip2 = one trip made by other user that day

If(trip2's mode is by motor vehicle AND same household 
AND specific purpose is the same as the main trip

AND the two trips happened around same time) then get the distance between the two destinations If(distance between is $<=1.5$ miles) \} then suggest to combine these trips

Rule 5: Multiple Households Multiple Users CARPOOL

- ANALYZES: MULTIPLE USERS IN DIFFERENT HOUSEHOLDS, MULTIPLE TRIPS, SAME DAY

- Purpose: Determines if multiple users could carpool to work based on the distance between their home locations and work locations.

RULE_preMultipleHouseholdsMultipleUsersCARPOOL()\{

If(destination is work related AND purpose is Work) then go to Multiple_Households_Multiple_Users_CARPOOL()

\}

MULTIPLE_HOUSEHOLDS_MULTIPLE_USERS_CARPOOL() \{

Retrieve destination2 and UserID2 of all other work locations close to this work place.

For each work location

Find the HomeID of User2's house.

Find distance between User1's and User2's home.

If(distance between homes $<=1.5$ )

then count the number of times they have carpooled

If(count is 0 )

then retrieve the name of User2

Suggest to User1 to carpool with User2 to work and back

\}

Rule 6: Transit

- ANALYZES: TRANSIT

- Purpose: Determines if a user can use a bus as an alternate mode of transportation on his/her way to work and back home.

RULE_preTransit\{ 
If(destination is work related

AND purpose is Work

AND starting point is home)

\}

then go to Transit_Rule()

TRANSIT_RULE() \{

Count the number of trips in which the starting point is work and the destination is home.

If(count $>0$ and mode is by motor vehicle)

then retrieve all of the bus stop GPS coordinates.

NumStopsNearDestination $=0$

NumStopNearStart $=0$

for each GPS coordinate $X$

Get the distance between the destination and $X$

if (distance is $<1$ mile)

then NumStopsNearStart++

Get the distance between the destination and $X$

if (distance is $<1$ mile)

then NumStopsNearDestination++

end for

If(NumStopsNearDestination $>0$ AND NumStopsNearStart $>0$ )

then Suggest the possibility of taking the bus

\}

Rule 7: Multiple Users Multiple Days

- ANALYZES: MULTIPLE USERS IN SAME HOUSEHOLD, MULTIPLE TRIPS, MULTIPLE DAYS

- Purpose: Determine if two or more people in the same household can run errands together in one trip.

RULE_preMultipleUsersMultipleDays\{

If(mode is by motor vehicle

AND purposes are valid combining trips

AND destination is not home

AND destination is not work)

\}

then go to Multiple_Users_Multiple_Days() rule 


\section{MULTIPLE_USERS_MULTIPLE_DAYS() \{}

Retrieve trips made by other users within a week period for the same purpose/specific purpose.

For each trip, $\mathrm{X}$

TRIP trip2 = new TRIP $(X)$

If(Household2 is equal to Household1

AND User2 is not User1

AND trip2 has valid purposes combining trips

AND trip2's mode is by motor vehicle

AND destination2 is not work

AND destination2 is not home

then find the distance between destination 2 and destination 1

If(distance difference is $<=1.5$ )

then suggest to Userl to take the trip with User2

Rule 8: Avoid Rush Hour

- ANALYZES: AVOID RUSH HOUR

- Purpose: Unless leaving work, avoid trips around rush hour, which is between 5:00pm and 6:00pm.

RULE_preAvoidRushHour \{

if (mode is by motor vehicle

AND starting point is not work)

then go to Avoid_Rush_Hour() rule

\}

AVOID_RUSH_HOUR()\{

If(trip has valid purposes for avoiding rush hour

AND trip start time is between $5 \mathrm{pm}-6 \mathrm{pm}$

AND it is a weekday)

\}

then suggest to leave before or after rush hour. 


\section{Chapter Four - Personal Travel Coach: Path Prediction Prototype}

This chapter describes a prototype module of the Personal Travel Coach that uses sophisticated real-time GIS analysis to predict a user's probable trip path and provides real-time alerts of any traffic incidents associated with predicted path. Path Prediction, also referred to as Route Prediction, records users' travel behavior, predicts their most probable immediate trip routes based on historical travel behavior data, looks ahead on each path of the route, and warns users of any incidents detected along these paths. This information is delivered to the users immediately and alerts them to take an alternate route before encountering the incident on their path. The Path Prediction Prototype has the ability to change travel behavior in real-time. A simplified illustration of an alert sent to a user in text message format on mobile phones is shown in Figure 10. The alert could also be given in many other formats including audio, picture, or video.

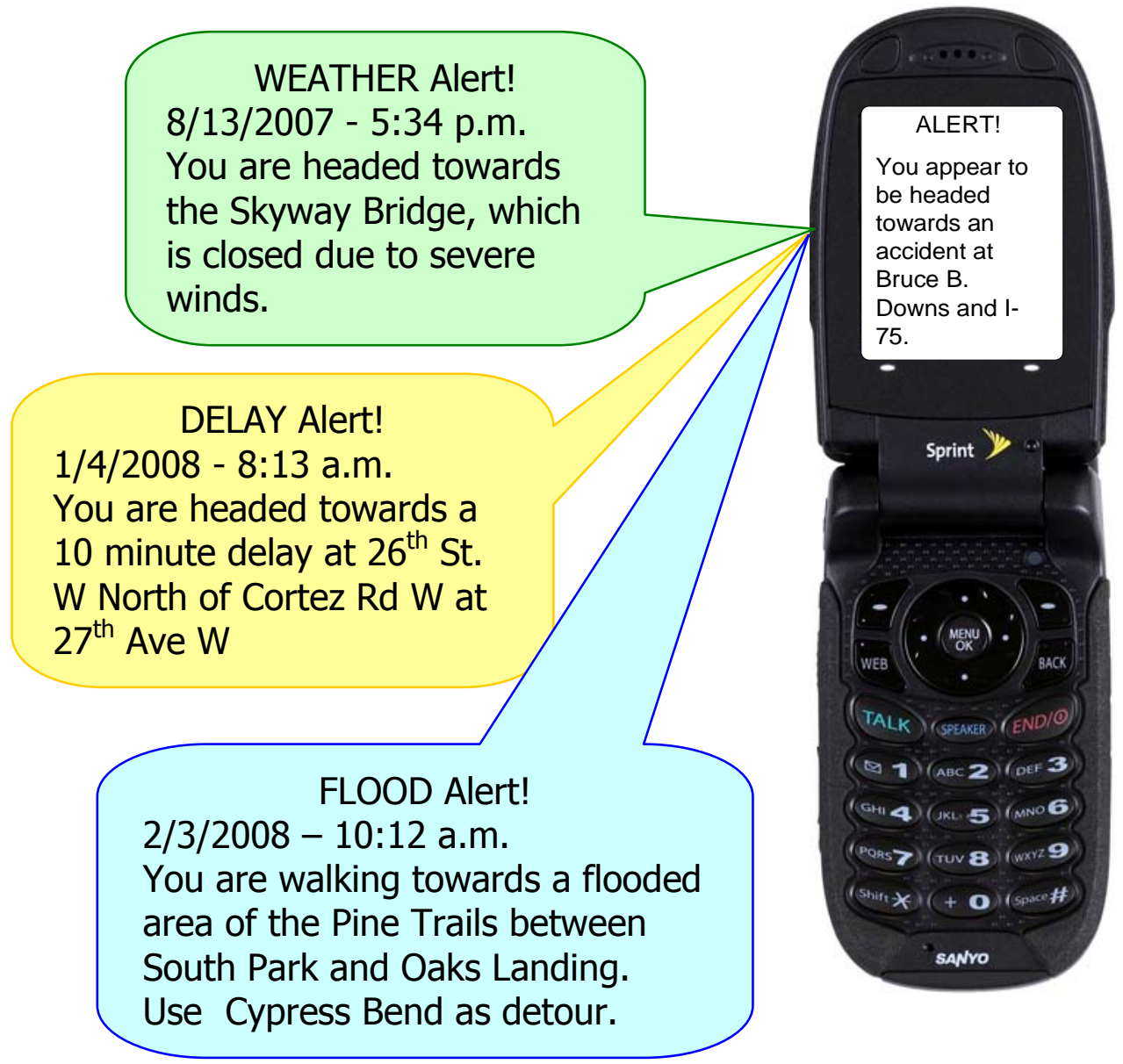

Figure 10 - Path Prediction Prototype - an illustration of text message alerts 


\subsection{Real-time Path Prediction and Traffic Incident Alerts}

The objective of advanced traveler information systems (ATIS) is to provide pertinent travel information to the consumer when, where, and how they need it. The goal is to enhance the experiences of the traveling public by informing them in a timely manner of delays, detours, schedules, and incidents so they can choose alternative routes instead of waiting for incidents to clear or waiting for trains, planes, or buses to arrive.

The emergence of GPS-enabled mobile phones provides many new opportunities for ATIS. Global commercial demand for location-aware services is forecasted to dramatically increase over the next several years. According to recent market research, the world population of GPS-enabled location-aware services subscribers will grow from 12 million in 2006 to a projected 315 million by 2011, and North American growth is projected from 500,000 users in 2006 to 20 million users in 2011 (27), (28). The Federal Communication Commission's (FCC's) enhanced 911 (e911) mandate, which requires cellular providers to be able to locate all emergency calls originating from mobile phones to generally within 50 to 300 meters of the actual geographic position, has driven the adoption of GPS-enabled mobile phones in the United States. Mass production of devices has continuously driven down costs for mobile phone hardware, including integrated GPS. These circumstances will provide an opportunity for large-scale deployments of the TRAC-IT software over the next several years. The Path Prediction algorithm is one feature of TRAC-IT that attempts to provide a direct incentive to the end user for recording their travel behavior and is considered an advanced form of real-time travel information.

Path prediction enables the TRAC-IT advisory system to analyze each trip the user travels without any manual input defining the intended destination from the user, thereby making it a completely passive system. It is expected that this system will be heavily used for day-to-day travel to locations that the user visits more than once. It can also be used to forecast imminent travel demands on the road network based on the results of many users' predicted paths. Utilizing mobile phones for route prediction and incident alerts in place of vehicle-embedded GPS devices or dedicated Personal Navigation Devices (PNDs) lowers the cost of equipment for the end-user as they utilize the already prevalent cell phone device. The majority of PNDs and embedded vehicle GPS devices do not track the user's travel behavior. Therefore, predictions based on personalized past behavior are not possible and the manual entry of the user's destination is required. Utilizing a cell phone opens the way for path prediction capabilities for transit and other nonmotorized modes of transportation where vehicular navigation devices are not available. 
The following sections discuss how the Path Prediction adds to customer convenience and changes travel behavior to ease congestion and increase reliability of traffic systems.

\subsubsection{Differences between Path Prediction and Expert System}

The previous chapter presented a main component of the TRAC-IT Personal Travel Coach, the Expert System. The Expert System collects and patterns travel behavior, analyzes the behavior against a set of rules, and suggests ways to decrease vehicle miles of travel and/or the elimination of trips, particularly the drive alone trips. Another component of the Personal Travel Coach that utilizes real-time location-based technologies to influence travel behavior is discussed in this chapter. Figure 11 shows the objectives of these two components as gradual long-term travel behavior change (Expert System) as compared against immediate behavior change which is relevant only for the current trip that the user is traveling in real-time (Path Prediction).

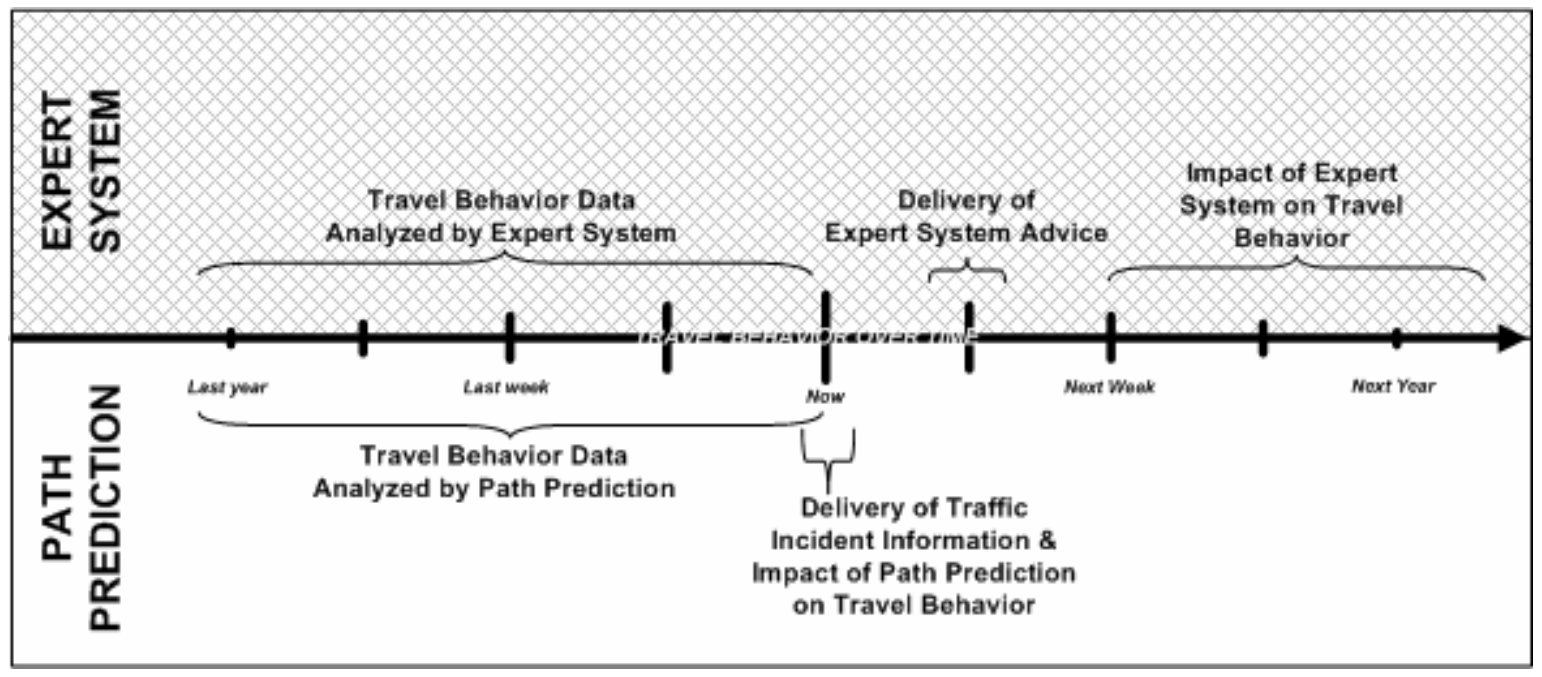

Figure 11 - Comparison between the Two Components of the Personal Travel Coach

The Expert System described in the previous chapter is purely rule-based and provides retrospective analysis to be considered by the TRAC-IT participant after their trips are completed. It is expected that the suggestion made to the users change their future travel behavior. In contrast, the Path Prediction Prototype seeks to provide users with information about incidents they may encounter along their travel path in real-time. In other words, the prototype provides suggestions based on the user's current location and circumstances. Path Prediction enhances TRAC-IT by using advanced GIS spatial queries to provide users with traffic incident information that could influence their behavior in real-time for trips they are actually recording using TRAC-IT. Researchers can use this tool to understand 
how real-time travel behavior changes as a result of pertinent information dissemination.

\subsubsection{Differences between Path Prediction and 511}

Since the Path Prediction Prototype is a real-time location-based application, as a TRAC-IT feature it is an improvement over existing traffic incident notification systems. Existing ATIS, such as 511, are primarily pull-based systems that require the user to contact the service and retrieve information for their current location through the phone or internet. Newer 511 features allow users to subscribe for incident notification services that are received in a text-message format to their cell phone. Although a useful service, it is not a location-based application. For example, 511 will repeatedly send incident alerts to subscribers even if the incidents are not in the user's path (i.e., the user is out-of-town or not traveling). Another disadvantage of the 511 service is the coverage area it provides users. For example, in the Tampa Bay Area, only the major interstates are monitored autonomously in real-time. Major arterials, trails, street networks - no matter how well-utilized by callers or web visitors - are not included in the system. Some advanced systems allow subscriptions to specific roadways, so the user will receive alerts only when incidents occur on these roadways. However, these systems send a deluge of irrelevant information since the subscription is tied to the roadway not to the user's physical location at relevant times. Some systems also limit the number of roadways to which the user can be subscribed and format the alerts as emails which are difficult or impossible to read on most mobile phones. Additionally, these systems require manual input of each roadway by the user when actively subscribing before alerts are received; the systems are not intuitive. It follows then that the service is counter-productive since the more roads the user signs up for, the more irrelevant information is received.

Path Prediction in TRAC-IT seeks to eliminate this excess information and to only present traffic incidents to users that are immediately relevant to their current geographic location based on their likely immediate travel path. In order to provide a passive always-on service to the user that requires no user input, a key element of this advanced functionality is the ability to predict the user's route based on personal travel behavior history collected by TRAC-IT.

\subsubsection{Differences between Path Prediction and Other Algorithms}

Previous research in path prediction methodologies has been focused on various creative ways to derive a user's trip destination. A Hidden Markov Model (HMM) was used to capture a sequence of driver actions that navigated a route and deduced the destination based on a present and next state transition model (29). The approach was successful for routine driving and for routes that deviated from schedules, such as for example, delivery or sales personnel routes. In contrast, the Path Prediction Prototype can transition between individual trip history and 
previous paths recorded by TRAC-IT to select probable routes and destinations by analyzing a variety of data collected. The driver is therefore guaranteed to get warnings related to the actual and/or neighboring routes. For incident alerts, accuracy in predicting an exact single future path is not as important as ensuring that incidents are detected on the set of most likely potential paths.

In past studies, prediction heavily relied on time-of-day approach. For example, the algorithm that used probability histograms taken over a period of three years for discrete daily time intervals matched the path of a bus passenger to one of five fixed bus routes (30) (31). Depending on the time of day, the algorithm anticipated that a bus will travel on a certain route (in a certain direction). If the bus deviated from this route, a new probability is calculated based on the remaining histograms. This approach was limited by its dependency on only five possible destinations and relied on bus routes that may change in the future or even daily. In comparison, TRAC-IT's predicting capabilities are not limited to a single mode of transportation; it can be used for walking or biking trips if the user traversed a repeated set of paths.

Currently, the trend in destination and path prediction focuses on probabilistic methodologies as in the use of histogram matching in comMotion research to conjecture a user's destination by incorporating HMM and a Bayes classifier to bolster predictions based on the individual's previously visited destinations (32). Two previous studies used data clustering to determine possible destinations. Markov models that are trained to predict a next most likely destination candidate based on recently visited destinations by using clusters of GPS data as inputs were used by Ashbrook and Starner (33). Research by Hariharan and Toyama focused on a location clustering algorithm that is calibrated and receptive to both scale and time (34). Liao et al. used a Bayesian network hierarchy outperforming a 2nd order Markov Model (35). Another study used point-by-point analysis method by clustering to match trips that appear to be similar (36). While these techniques may provide good prediction results in their respective tests, each has a large degree of computational complexity that limited the scalability of such systems for a large number of mobile phones. Unlike these techniques, TRAC-IT's Path Prediction algorithm, in its simplest form, does not use probabilities to determine a traveler's destination. It makes indirect use of simple trigonometric and calculus calculations to perform queries related to positions given by GIS operations. These operations are referred to as spatial queries implemented using GIS products, such as those produced by ESRI (37). Spatial queries utilized in the Path Prediction algorithm can also be individually optimized allowing for a highdegree of scalability necessary to provide real-time services to a large population of active mobile phones. In addition to the ArcObjects Java API for ArcServer used to implement path prediction, ESRI provides programs such as ArcView that allows the research to be manifested in a visual and illustrative manner (38) (39). Geometries representing the user's physical travel history can be analyzed and tested in a visually-enhanced rather than a trial-and-error approach. Though 
statistical probabilities can potentially improve Path Prediction accuracy, they were not incorporated in this study to maintain system simplicity and scalability.

Another advantage of Path Prediction is that it does not require knowledge of the underlying street network. Instead, the past travel behavior of the user serves as the street network that is specific to that particular user. The resulting system is an extremely efficient algorithm that utilizes unprocessed GPS data (other than the Critical Point algorithm, discussed in detail in the TRAC-IT 3 Final Report) and simple spatial queries to provide real-time services to the GPS-enabled cell phone user.

\subsection{Methodology used for Path Prediction Prototype}

The prototype uses traveler past history to determine their likely future destination. To process a participant's travel history for path prediction, an initial application, referred to as the Path Maker algorithm, retrieves and catalogs the user's travel behavior. Once these statistics and data are archived in a GIS database (using ESRI ArcSDE and SQL Server 2005), the path prediction algorithm then executes in real-time and anticipates the user's trip route based on past travel patterns. If an incident is detected within one of the user's potential paths the algorithm can send any warnings related to their route. Incident information can be retrieved from many potential data sources and formatted into a geographic element, including a single point, line, or polygon covering a large geographic area, which can be detected by the algorithm.

\subsubsection{The Path Maker Algorithm}

The Path Marker algorithm (Figure 12) is responsible for developing and maintaining an archive of user trip records. It queries a SQL database table for trip coordinates categorized by trip ID, user ID, and trip time. From the trip coordinates, the application uses the ArcObjects Java API to construct a geographic and geospatially accurate polyline. A polyline is a set of straight line segments connected to form a larger path (not unlike connect-the-dots). Consequently, it requires a minimum of two points to be considered a polyline. ArcObjects is a set of GIS software components, namely APIs, used for spatial and geographic calculations providing the developer with objects that perform a multitude of spatial and geographic calculations. 


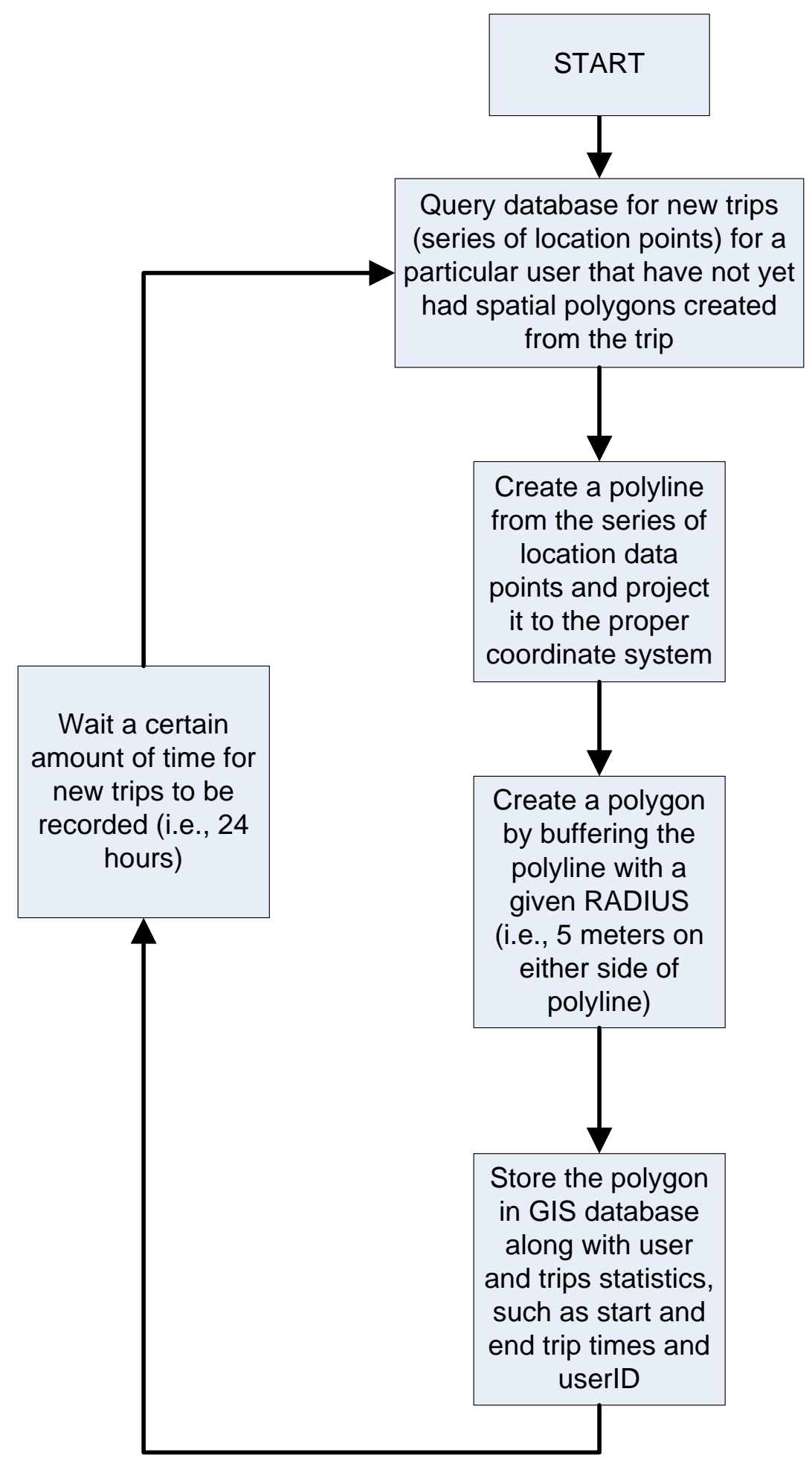

Figure 12 - Flowchart for Path Maker Algorithm 
Once the coordinates have been queried and retrieved and the polyline assembled, the Path Maker algorithm buffers the polyline with a 5 meter radius to create a polygon. Figure 13 shows a section of the polygon-buffer network topology for trips taken by multiple users. This polygon is then stored in the database server using ArcSDE along with its statistics, such as trip and user id, and a timestamp that consists of the trip's start and end time. Storing polygons is important to path prediction because it is more efficient to perform spatial calculations using area geometries in a batch process once rather than reprojecting and buffering each polyline repeatedly at runtime for each new spatial query. Additionally, bounding boxes can be utilized to approximate polygons in order to further enhance system performance and scalability.

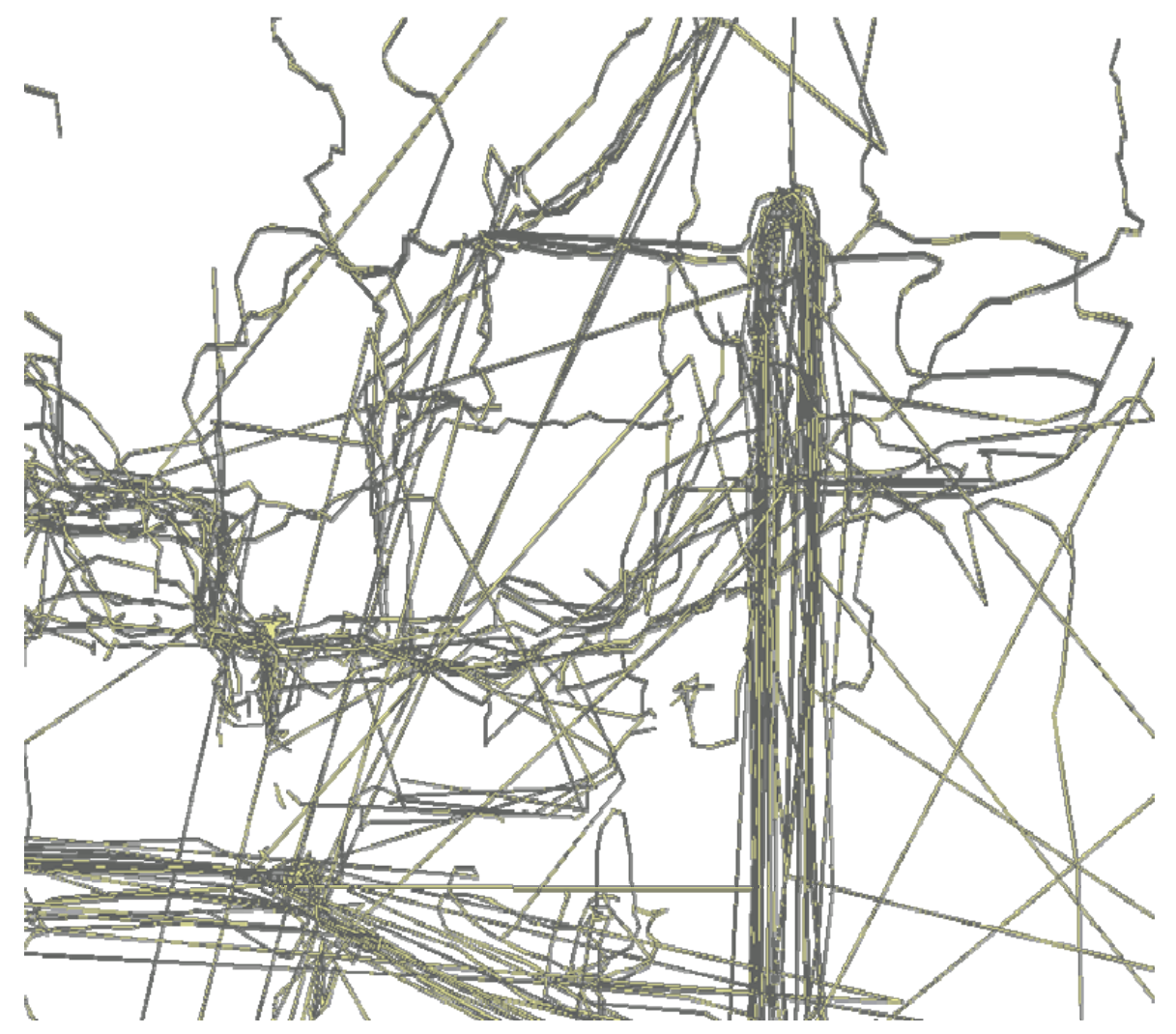

Figure 13 - Polygon Network Topology for User Recorded Trips

\subsubsection{The Path Prediction Algorithm}

After the GIS database is populated with polygons, the algorithm searches for records to compare against a user's current route. A flowchart illustrating the Path Prediction algorithm is shown in Figure 14. A buffer on the server is used to hold the real-time coordinates from the mobile phone as they arrive. As an example, the buffer size for this illustration is set to five coordinates. 


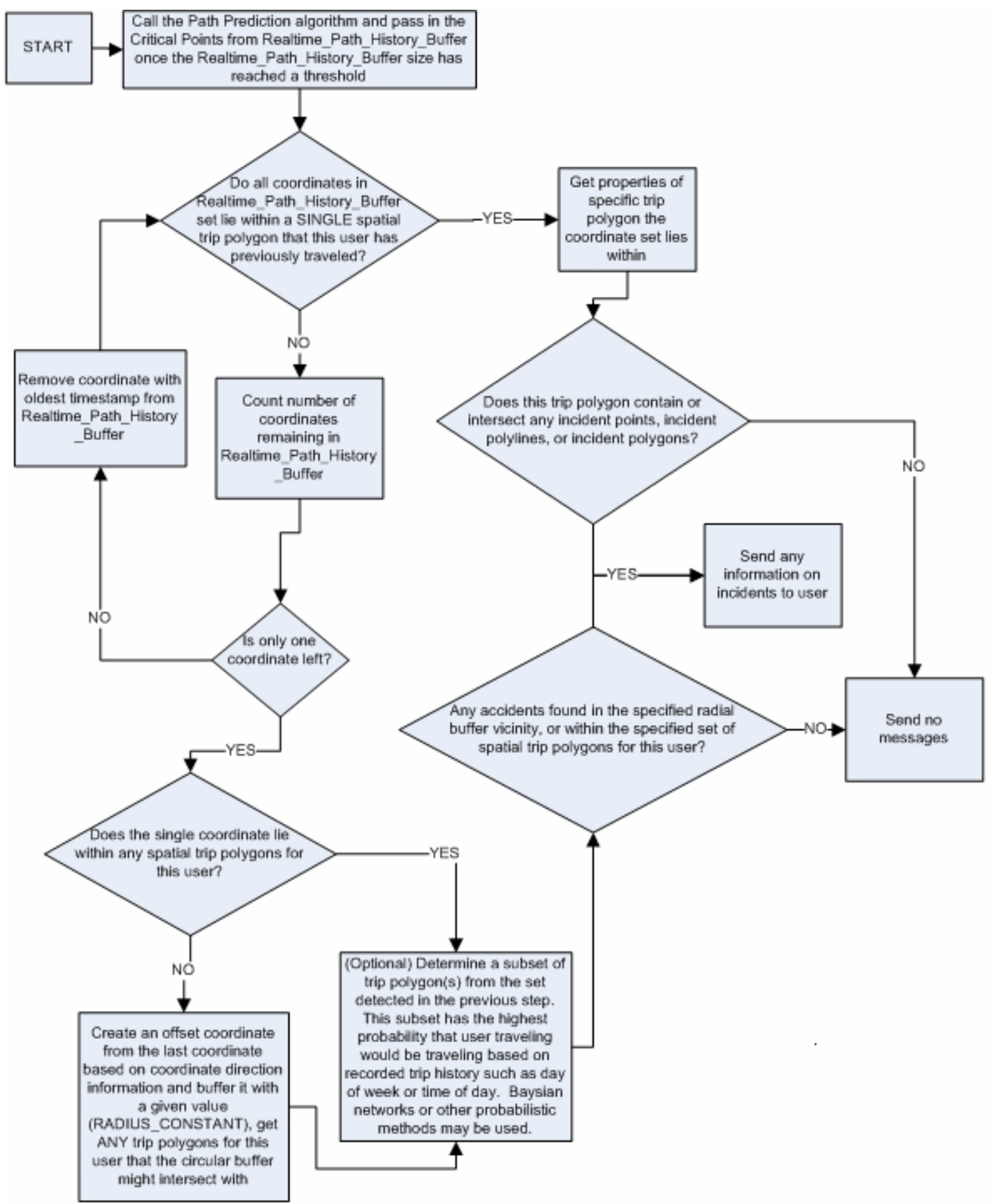

Figure 14 - Flowchart of TRAC-IT Path Prediction Algorithm

When the user starts recording a trip with his GPS-enabled mobile phone, the path predictor awaits receiving the first five coordinates directly from the user's phone. The algorithm then forms a polyline using these coordinates and performs a polyline-in-buffer spatial query against the ArcSDE database. For this query, the polyline must be totally encompassed within a polygon buffer. If multiple buffers 
are retrieved from the query, the algorithm refines the results by comparing the traveler's user ID to those of the polygons and eliminates any polygons with a different user ID. The best case scenario, but certainly least likely, is that only one record is returned. The worst case scenario is that no polygon records are returned.

When either multiple buffers or no buffers are returned in the initial spatial query, the algorithm attempts to refine the search. Initially, the first coordinate in the five coordinate set will be eliminated and the polyline-in-buffer query will be performed again in a recursive fashion until only a single buffer is returned or one coordinate remains. This is specifically performed because the success of a polyline-in-buffer spatial query requires a polyline be completely confined within the polygon. This situation may occur if the user's current trip begins recording before his or her past trips, whereby the polyline might not be adjacent to other polygon records. If only one coordinate remains as a result of the recursive method, the last coordinate is used to create projection of the user's likely next position. A new coordinate is created by adding an offset in the direction of the heading of the last coordinate to obtain a new point. A circle with a 10-meter radius is then created around this point, and an intersection query against the ArcServer polygon database is performed to retrieve any polygon records within the circular vicinity. This situation can occur if a polygon record was never created for a user's path or if the database is sparsely populated. The procedure is typically performed to give the user immediate feedback about any hazards within their vicinity rather than send them no information at all. The algorithm will be called again with a new set of five coordinates, and the process will be repeated giving the user new and improved updates.

\subsubsection{Sources of I ncident I nformation}

There are many potential sources of incident information that can be formatted for detection by the path prediction algorithm, including real-time traffic incident information. The algorithm can be used to detect any spatial feature that overlaps with the user's predicted path, include zones or lines that represent hazardous events or areas. These spatial features can be generated from potentially any source of data where a specific location can be assigned to the event.

The path prediction algorithm was tested against a synthetic dataset created by TRACIT researchers that represented traffic incidents at particular points, represented by latitude and longitude. In these simulations, when there was an incident in the user's predicted path, the TRACIT was able to successfully trigger an alert to be delivered in the form of a text message to the mobile phone. Future enhancements planned include delivering driving directions for alternate routes and verbal feedback to the user. 
In an attempt to test the system with real-time traffic information, TRACIT researchers identified Traffic.com (formerly Mobility Technologies, Inc.) as the company that manages the sensor systems on highways in the Tampa Bay area under a contract by the Florida Department of Transportation (FDOT). These sensors detect vehicle speeds and are used as part of the 511 Tampa Bay system to report travel speeds to the public through a website and phone system. The research team contacted Traffic.com in mid-2005 in an effort to gain programmatic access to real-time traffic data. The team was first pointed to a website that allows users to browse and download data using a web browser, but does not allow programmatic access via software to the data. When it was apparent that access to this website alone would not be sufficient, the research team requested access to the database system that was supplying the data to the web page. Traffic.com stated that a new contractual agreement was necessary between USF and Traffic.com before access to the database would be granted to the research team. After lengthy negotiations between USF and Traffic.com, in late 2006 discussions with terminated were Traffic.com since no agreement between USF and Traffic.com could be reached that would allow USF access to the sensor systems that provide the speed data for highways in Tampa.

Since this time period, Traffic.com has been purchased by Navteq. Therefore, future efforts to collaborate with Traffic.com may be successful if corporate strategies or management within the company have changed. Future work will also target interfacing TRAC-IT with other real-time ATIS systems such as those in south and central Florida.

\subsection{Sample Scenarios of Path Prediction}

There are several specific scenarios that the Path Prediction algorithm is designed to handle in order to provide accurate predictions to detect and deliver incident information. This section discusses several of these scenarios. In the illustrations, the polygons are spatial trip buffers, or polygons, that were created in the Path Maker application and represent previously recorded trips by a specific user. In a real situation, the user would have many of these polygons associated with their account. 


\section{Scenario 1: Real-time trip data are fully contained within a single spatial trip polygon}

In Scenario 1, Figure 15, five critical points were recorded by the mobile phone and passed to the Path Prediction Algorithm. The direction of travel is from left to right. Since the real-time path is fully contained within a single buffer (shaded polygon), this polygon is found to be the predicted path for the real-time travel of this user. For incident detection, the shaded polygon is checked for intersection with any incident shapes (i.e., points, polylines, or polygons) and, if there is an intersection, the user is notified.

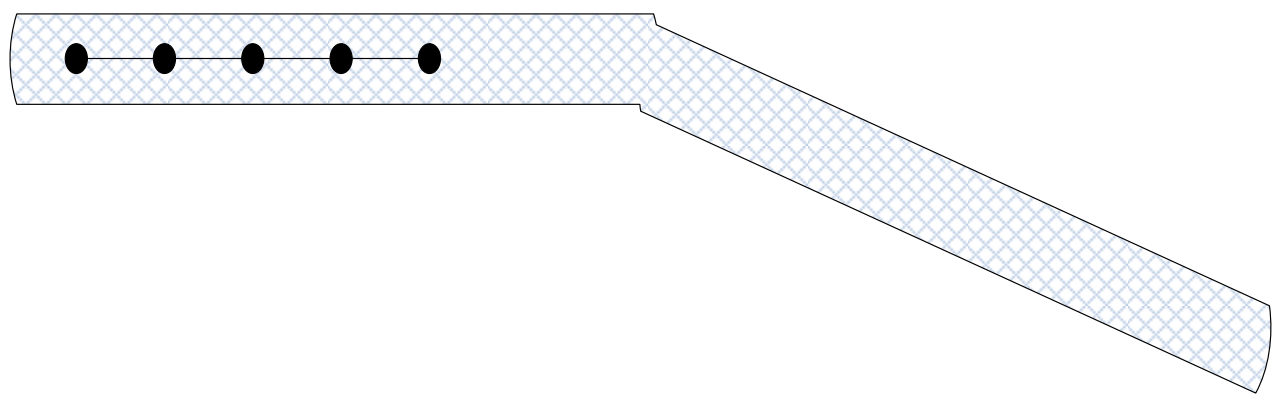

Figure 15 - Real-time Trip Data Fully Contained in a Single Polygon

\section{Scenario 2: Real-time trip data partially intersect a single spatial trip polygon}

In Scenario 2, Figure 16, five critical points were recorded by the mobile phone and passed to the Path Prediction Algorithm. The direction of travel is from left to right (i.e., the triangular coordinates were recorded first). Since the first two coordinates did not fall within a trip polygon, they were removed one-by-one until the remaining polyline lied completely within the trip polygon. This polygon predicts the users immediate travel path. The algorithm looks to see if any incident shapes (i.e., points, lines, or polygons) intersect with this trip buffer, and, if it does, the user is notified.

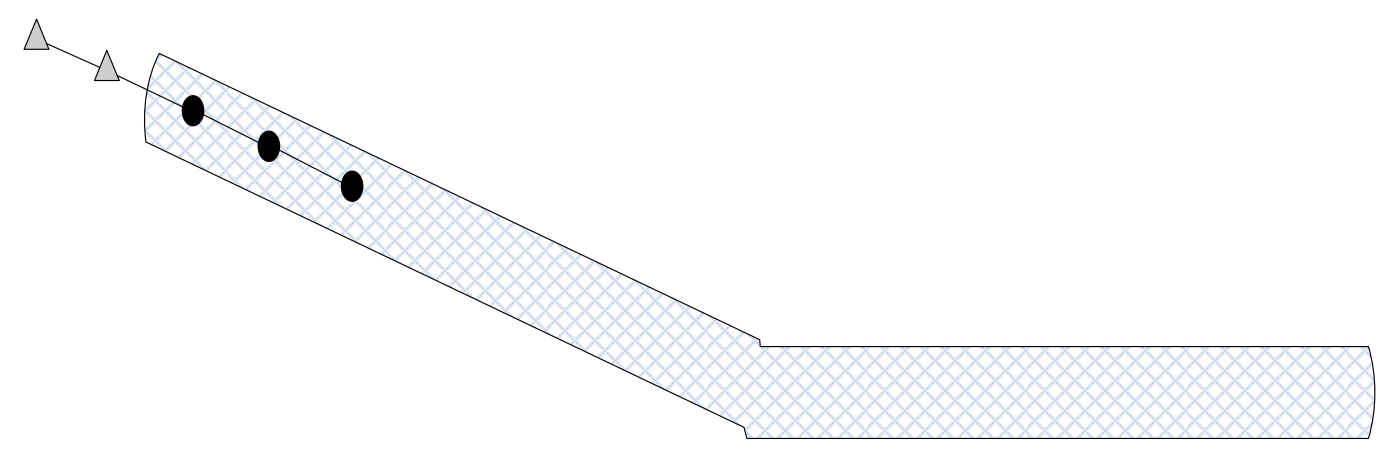

Figure 16 - Real-time Trip Data Partially Intersect a Single Polygon 


\section{Scenario 3: Real-time trip data does not intersect any spatial trip polygons}

In Scenario 3, Figure 17, five critical points were recorded by the mobile phone and passed in the Path Prediction Algorithm. The direction of travel is from left to right (i.e., the triangular coordinates were recorded first). Since the first entire trip polyline did not fall within a trip polygon (even after the oldest points are removed one-by-one), an offset coordinate (circular point at center of radius) is created based on the direction of travel of the last recorded point (triangular point immediately to the left of circle). A spatial radius buffer (shaded circle) is then projected around this offset coordinate. In this scenario, the radius buffer intersects with a trip polygon, so this trip buffer is used to predict the user's immediate Path. The algorithm looks to see if any incident shapes (i.e. points, lines, or polygons) intersect with this trip buffer, and, if it does, the user is notified.

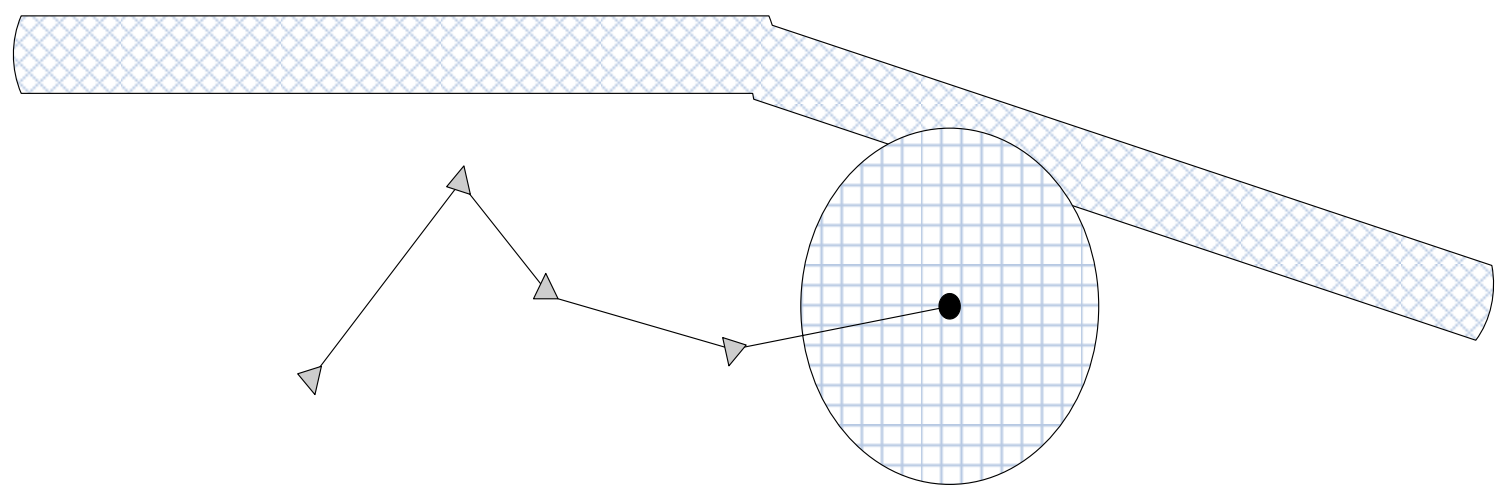

Figure 17 - Real-time Trip Data Not I ntersecting Polygons

\section{Scenario 4: Real-time trip data intersect multiple spatial trip polygons}

In Scenario 4, Figure 18, five critical points were recorded by the mobile phone and were passed in the Path Prediction Algorithm. The direction of travel is from left to right (i.e., triangular points were recorded first). Since the real-time path intersected two buffers (white and shaded), the oldest points (triangular points) were removed one-by-one until the newest location data polyline (circular points) lies completely within a single polygon (shaded polygon). In this situation, the shaded polygon is used to predict the user's immediate path. The algorithm looks to see if any incident shapes (i.e., points, lines, or polygons) intersect with this trip buffer, and, if so, the system notifies the user. 


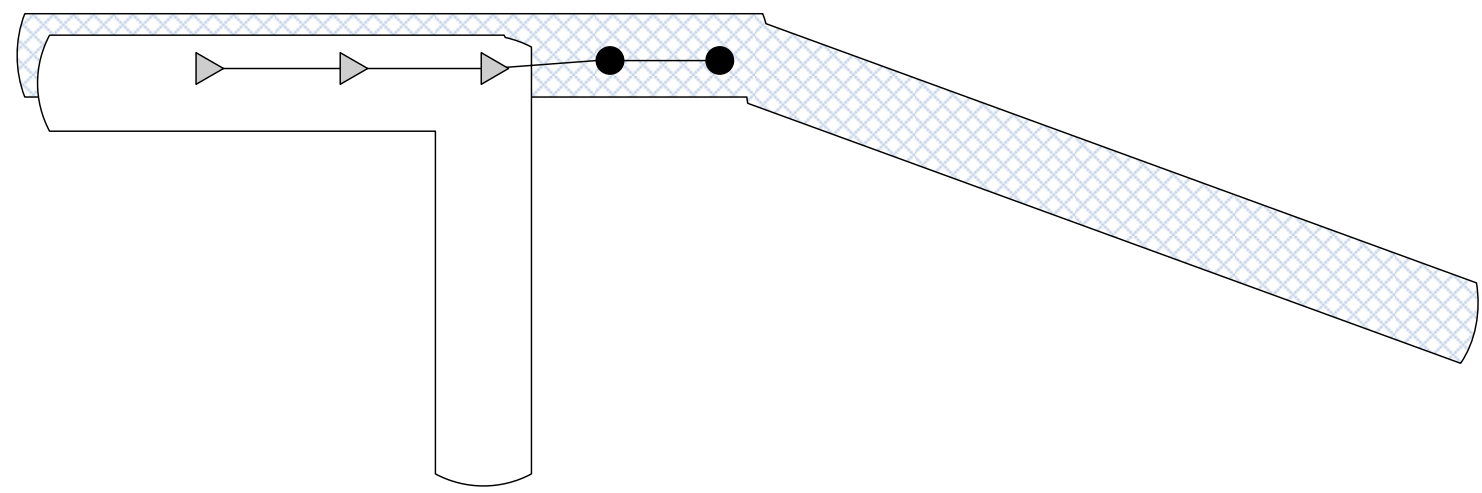

Figure 18 - Real-time Trip Data Intersect Multiple Polygons

Scenario 5: Real-time trip data are fully contained within multiple spatial trip polygons

In Scenario 5, Figure 19, five critical points were recorded by the mobile phone and were passed in the Path Prediction algorithm. The direction of travel is from left to right. Since the real-time route is fully contained within two buffers, probabilistic methods can be used to determine the most likely route taken by the user. Schedule data from trip polygons such as trip day of week or time of day may also be used. For incident detection, all spatial trip buffers that the real-time data is fully contained within are checked for intersections with any incident shapes (i.e., points, polylines, or polygons) and, if there is an intersection, the user is notified.

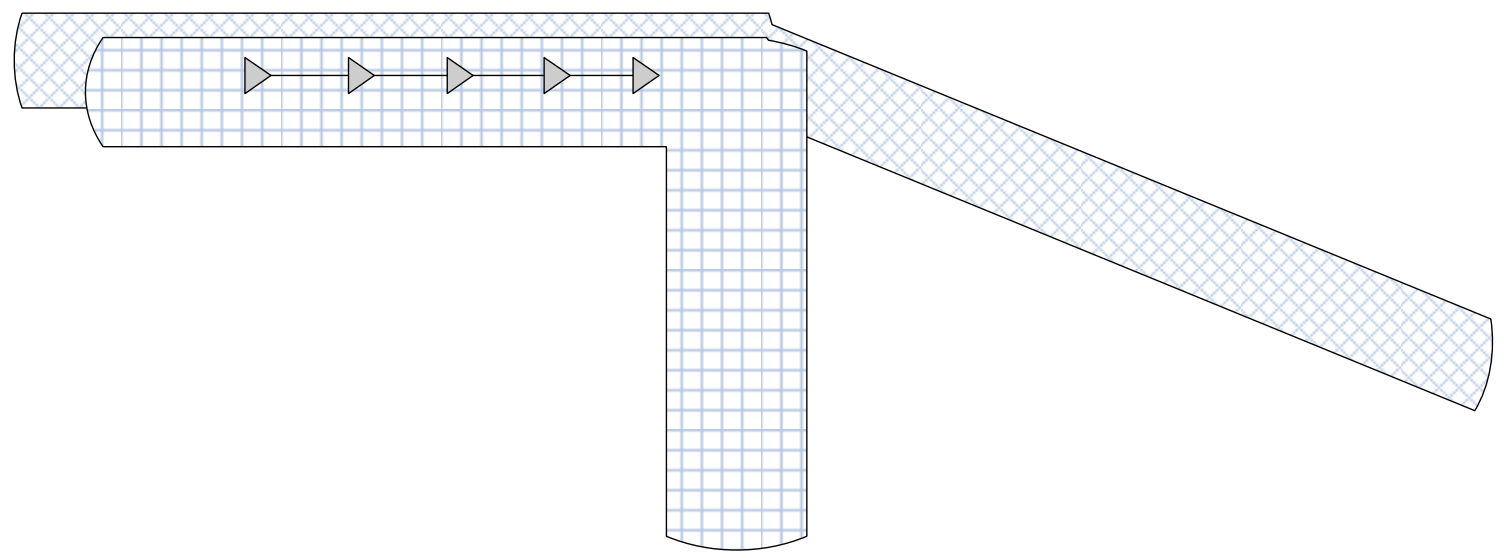

Figure 19 - Real-time Trip Data Fully Contained Within Multiple Polygons

\subsection{A Simulation of TRAC-IT Path Prediction}

A simulation was performed using coordinates from a randomly-selected trip from the TRAC-IT Phase 3 field tests. The trip was recorded on September 6, 2007, and trip buffers were recorded prior to that date. This simulation was conducted using raw assisted-GPS data produced by the cell phone. No filtering or corrections, 
other than the Critical Point algorithm, were used. The coordinates from the trip (referred to hereinafter as TripID 77) were input into the algorithm as though TripID 77 was being recorded in real-time. Since the trip end is known, one goal of the simulation was to determine when the algorithm would first identify a buffer (Buffer-77) with the same destination (as labeled by the user with the TRAC-IT user interface) as TripID 77 as a potential real-time user path. If Buffer-77 is detected as having the same destination as TripID 77, the user is alerted for any incidents that lie on the path between their current position and their destination. Other buffers with different destinations may have been returned along with Buffer-77 which could have potentially resulted in additional alerts sent to the user not relevant to the destination of TripID 77.

Another goal was to determine how long it would take to return a single buffer that had the same destination as TripID 77 . This exercise represents the correct prediction of a single path that can eliminate false positives for incidents detected on other routes initially returned as possible match candidates but did not have the same destination as TripID 77.

\subsubsection{Simulation Analysis}

The simulation represented by Figures 20, 21, 22, 23, and 24 took 29 seconds to execute on the server. A total number of 56 iterations were performed before the results were narrowed down to the single buffer. TripID 1642 had the same destination as TripID 77. However, it only took 40 iterations for the correct buffer TripID 1642 to be returned along with other buffers that contained different logical destinations (as labeled by the user in the TRAC-IT user interface). The estimate for the server to receive a set of five critical points from the mobile phone was 44 seconds based on a number of recorded trips. In a real-world test approximately 2 minutes and 7 seconds would have elapsed before the Path Prediction Algorithm returned TripID 1642 as a possible match. An estimate of 3 minutes would have elapsed before the algorithm narrowed down the results to the single correct destination. For comparison, TripID 77 was 20 minutes in duration from the start to end time. For clarity, only five iterations are shown in the simulations figures as several iterations had the same detected buffers. 


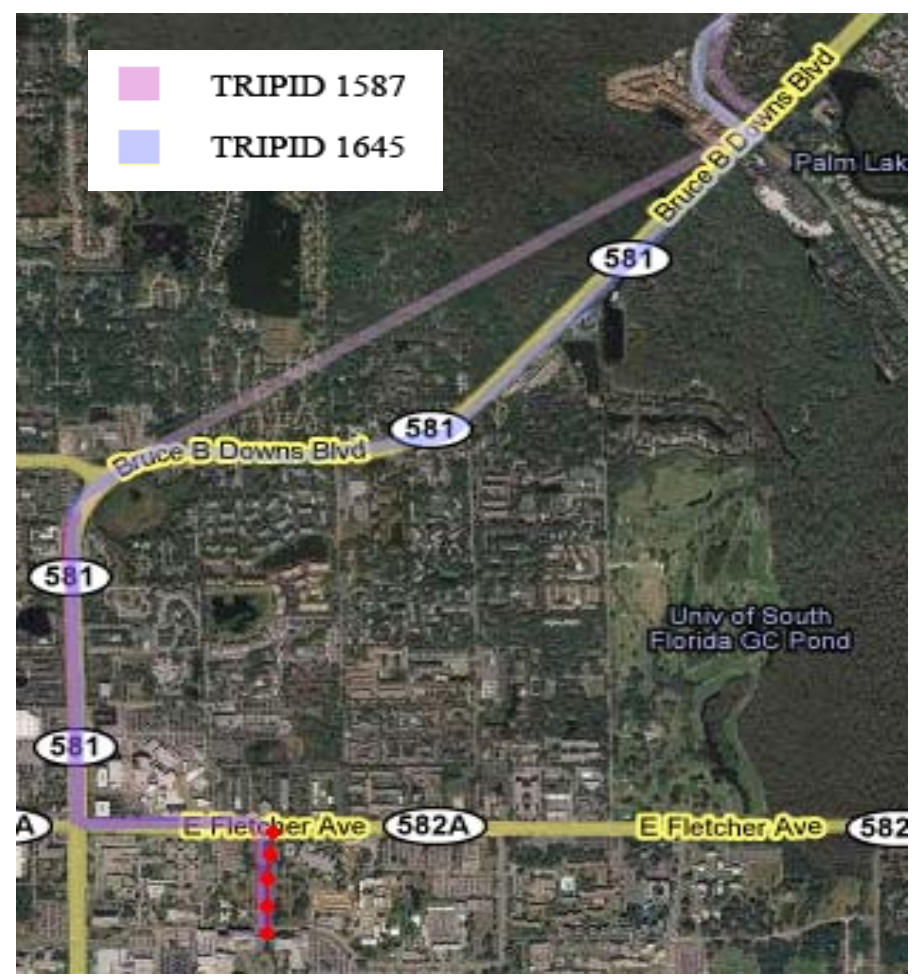

Figure 20 - I teration 1 of Path Prediction Algorithm Simulation

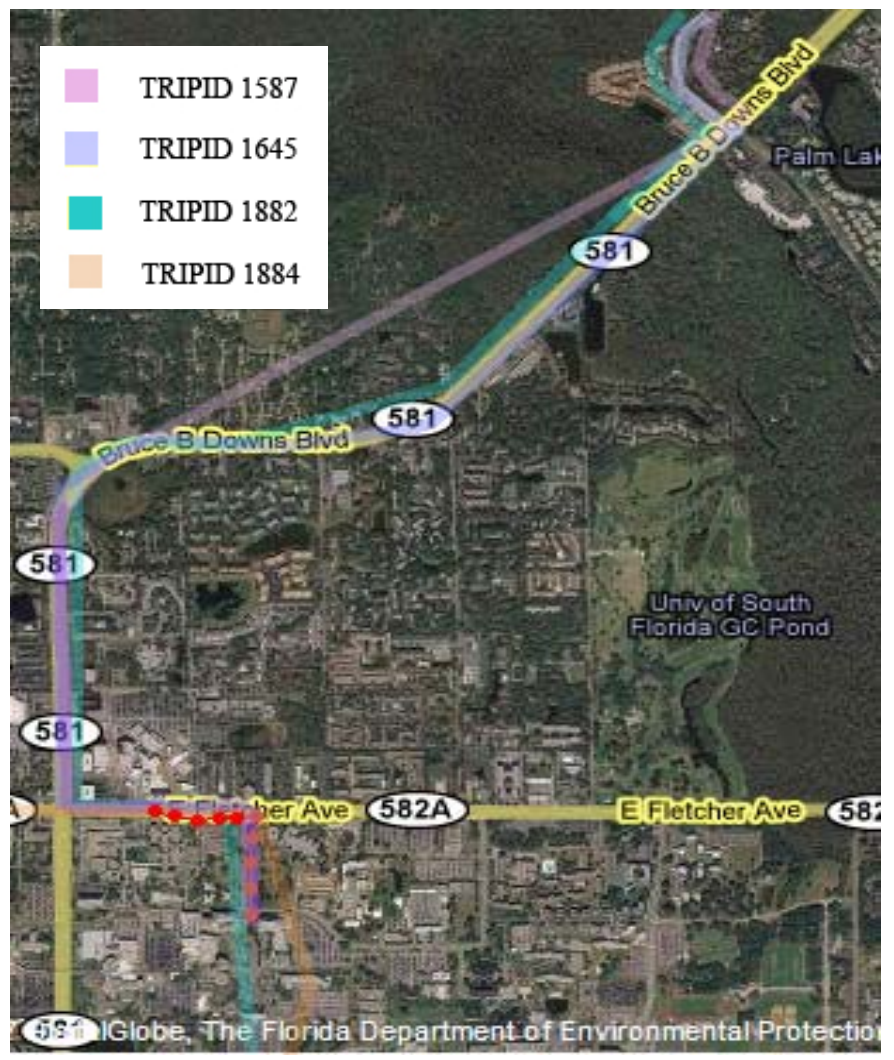

Figure 21 - I teration 33 of Path Prediction Algorithm Simulation 


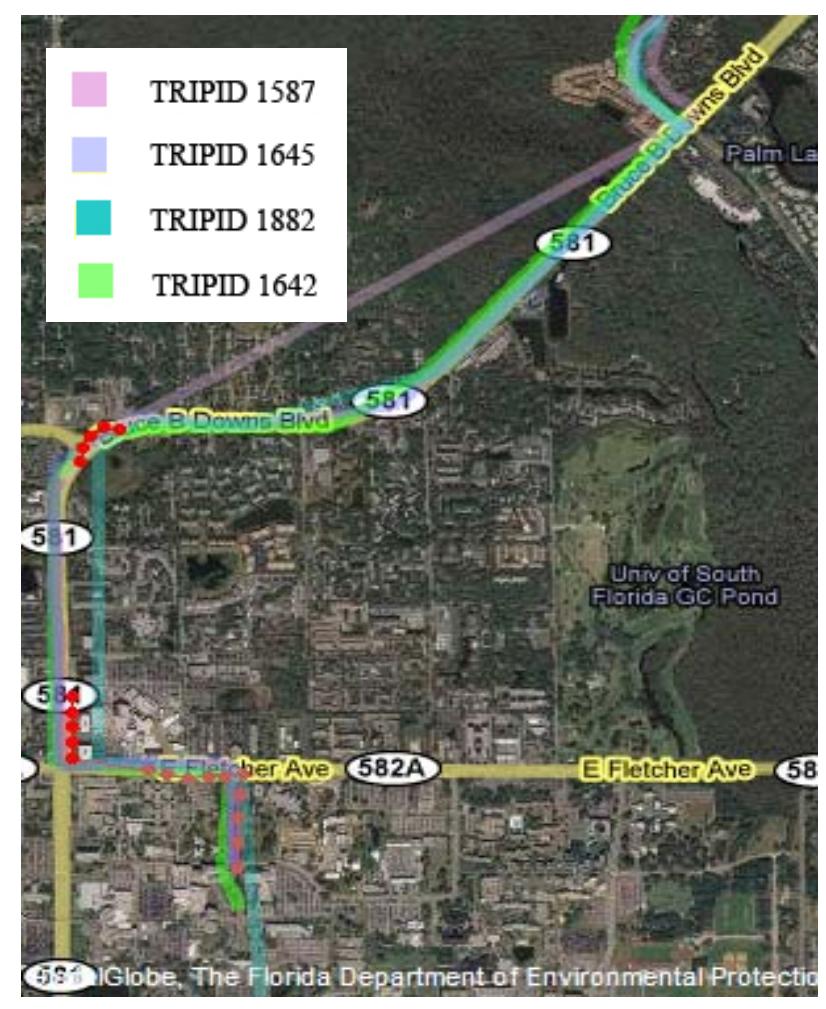

Figure 22 - I teration 40 of Path Prediction Algorithm Simulation Showing First Appearance of Correctly Predicted Path, Tripl D 1642

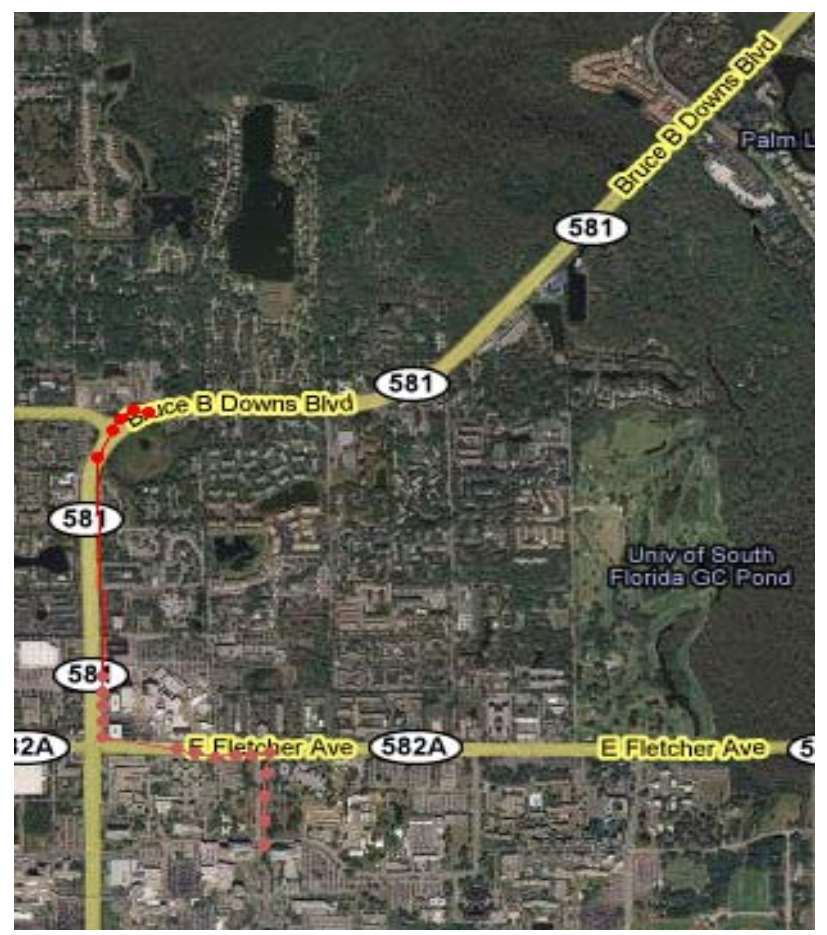

Figure 23 - Iteration 51 of Path Prediction Algorithm Simulation 


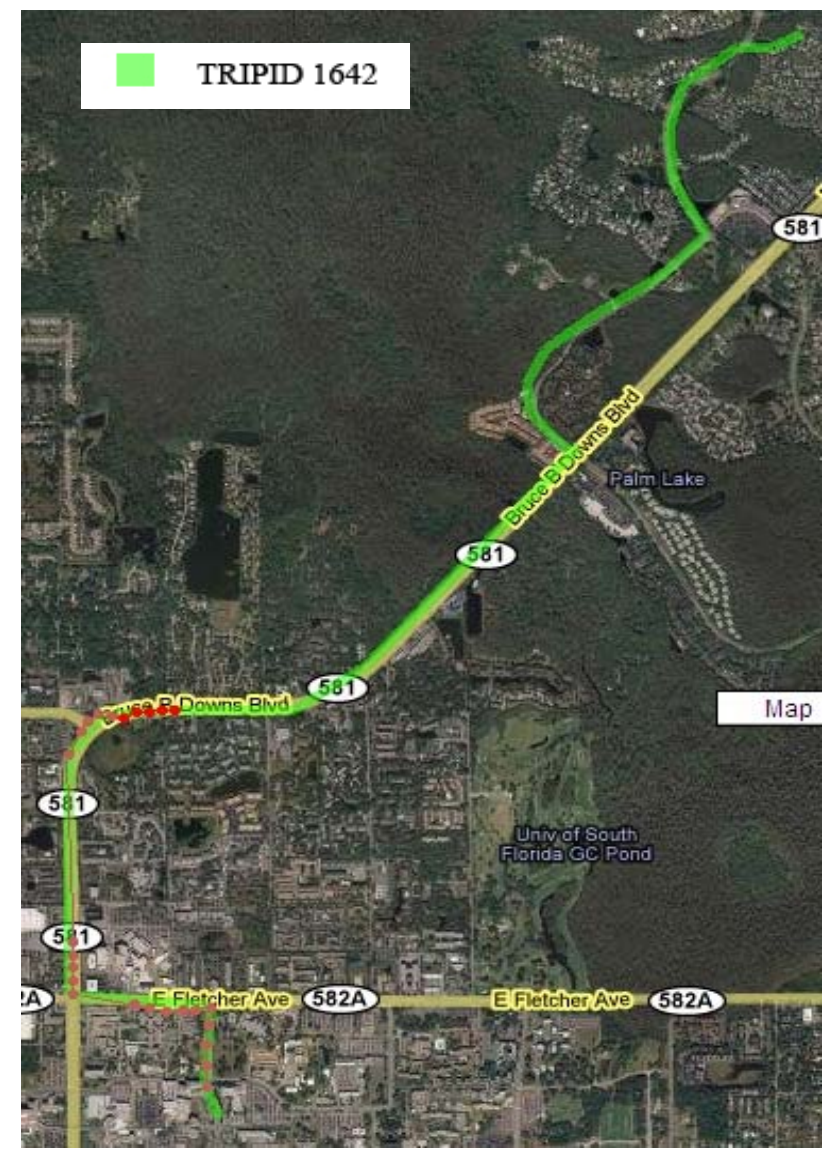

Figure 24 - Iteration 56 of Simulation Showing a Single Path, TripI D 1642, Correctly Predicted Destination

\subsubsection{Discussion}

Looking at the first iteration; it is apparent that few buffers were detected initially because the user started his trip on a side street. Occasionally, a mobile phone may take a few moments before getting a GPS fix, which explains why only two buffers were initially detected. As the user moved on to a major road, the number of buffers detected doubled. It should be noted that the buffers returned in each subsequent step overlap the correct buffer for a significant portion of the trip. Even though these incorrect buffers contain destinations that are logically different (e.g., the final destination is labeled Publix instead of Home), many of the destinations for these buffers lie within a quarter of a mile of the correct destination. Therefore, it is likely that any traffic alerts issued for the user would still have been relevant to their current real-time path. The user also would have received these alerts prior to committing to the main road that travels north and therefore could have changed directions and taken an alternate route if desired.

Another important observation is that some buffers are skewed or appear nonsensical. In comparing Buffer 1587 in Figures 20, 21, and 22 to its 
surrounding streets, it is noted that it is somewhat misaligned. This is attributed to GPS dropout, where the mobile phone loses a GPS fix and then re-establishes it some time later. GPS drift, or slight errors in GPS that result in small deviations in the recorded position, can cause the real-time trip to fall outside of past trip buffers and result in no buffers being detected (Figure 23). It can also be seen in Figure 24 that the correct TripID 1642 would have been detected in the first iteration of the algorithm represented by the first 5 vertical critical points if the GPS paths from TripID 77 and Trip ID 1642 were slightly closer together. Future research should focus on removing or repairing trips with GPS dropouts and experimenting with larger geographic buffer sizes that could better accommodate these errors.

The results obtained from this single simulation, though not definitive, are promising. They demonstrated that the TRAC-IT Path Prediction approach delivered the anticipated and desired results using simple GIS queries which can be scaled to a large number of mobile phones and can run in real-time. While further testing and refinement are required, the research shows that there is great promise for real-world implementation of the algorithm. 


\section{Chapter Five - TRAC-IT Phase 2 Survey Implementation}

As previously mentioned, this study used the TRAC-IT system developed in Phase 3 of the research series to collect travel behavior data from a sample of households over at least a one-week period. After the collected data were instantly transferred to a database, they were analyzed by the Expert System discussed in Chapter 3, and appropriate travel suggestions were generated. This chapter describes the survey instrument and protocol, the method selected to verify the validity of the data collected from mobile phones, the suggestions generated and methods of feedback delivery to participants. This chapter also documents recruitment issues and deployment options as encountered by the research team throughout TRAC-IT implementation efforts.

\subsection{TRAC-IT Survey Instrument}

The GPS-enabled mobile application developed in Phase 3 is able to record the route taken by the survey participant, regardless of mode, by calculating a series of GPS fixes, each representing the distinct location of a participant at a certain time while carrying the phone. TRAC-IT can record GPS data at a rate of up to one GPS fix per second. Data collected by the survey instrument in the form of GPS data include trip start and end times, trip duration, travel route, trip distance, trip origin, and trip destination in terms of latitude and longitude coordinates. Information that is difficult to extract from GPS data is input by the participant selecting options from the TRAC-IT user interface on the mobile phone. The information manually selected includes travel mode, trip purpose, trip origin and destination, and if applicable, vehicle occupancy and whether the participant was the driver or the passenger in the vehicle.

\subsubsection{TRAC-IT User Interface}

A detailed description of the TRAC-IT mobile phone and server system is available in the TRAC-IT Phase 3 final report. An overview is briefly summarized in the following sections as relevant to TRAC-IT Phase 2 activities. Sample screens from the TRAC-IT mobile phone user interface are shown in Figures 25 and 26. 


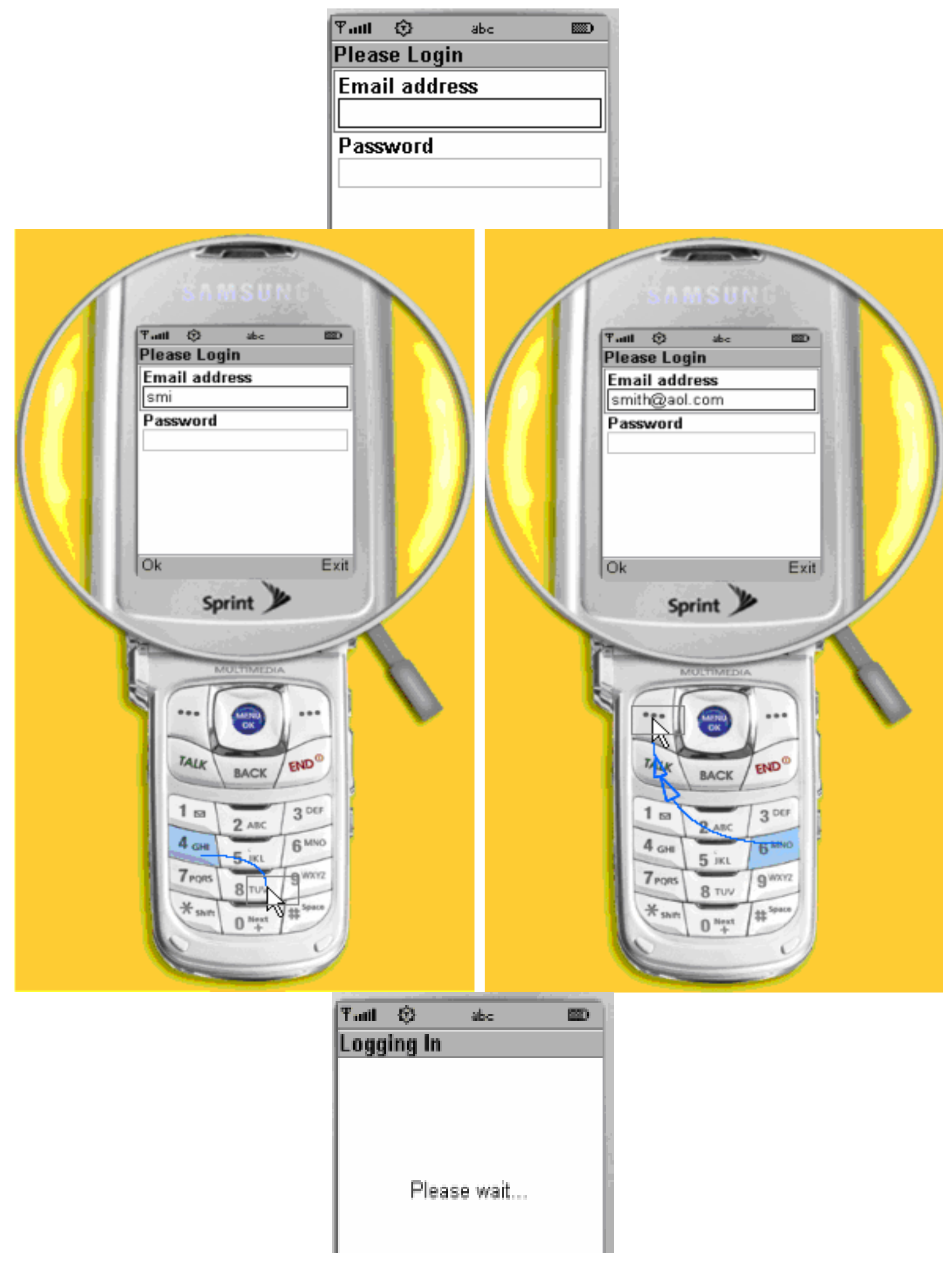

Figure 25 - TRAC-IT Login Screens 

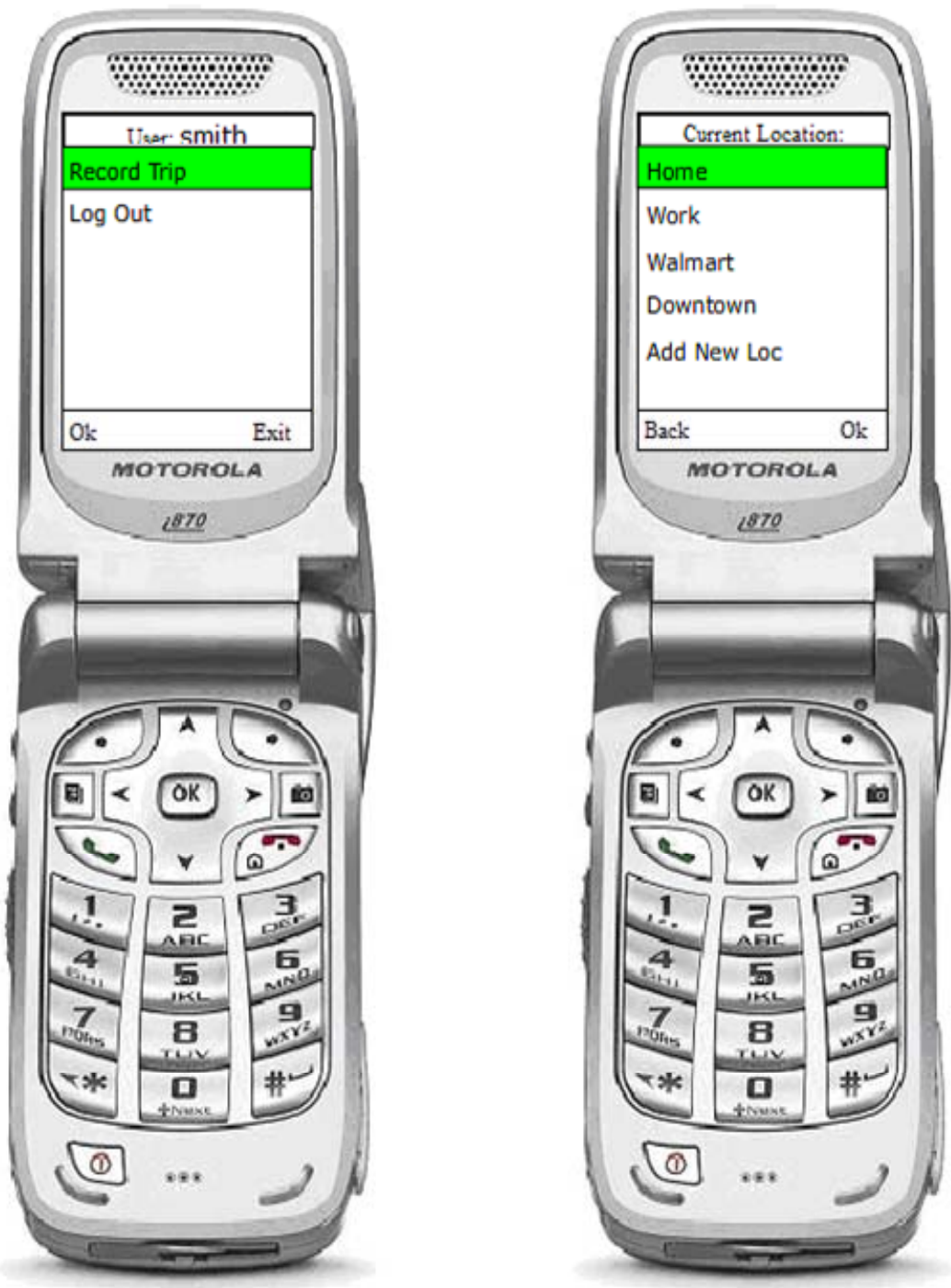

Figure 26 - Begin Trip and Current Location Screens 
The user has to select one of the following trip purposes, as well as a subcategory if applicable, when prompted to do so:

1. Work

2. School or Religious

$\checkmark \quad$ Go to school

$\checkmark \quad$ Go to a religious activity

$\checkmark \quad$ Go to the library: school related

3. Medical or Dental

4. Shopping and Errands

$\diamond \quad$ Buy goods: groceries, clothing, house needs, gardening needs, etc.

$\checkmark \quad$ Buy services: post office, bank

$\checkmark \quad$ Car services: pump gas, car maintenance

$\checkmark \quad$ Personal or family business

$\checkmark \quad$ Pick up or drop off an item: dry cleaners, video rental, etc.

5. Social and Recreational

$\checkmark \quad$ Go to the gym, exercise, play sports, etc.

$\diamond \quad$ Rest, relaxation, or vacation

$\checkmark \quad$ Visit friends or family

$\checkmark \quad$ Go out: entertainment, theater, sports event, bar, etc.

$\checkmark \quad$ Visit public place: historical site, museum, park, etc.

6. Transportation of Someone or Myself

$\checkmark \quad$ Pickup someone

$\checkmark \quad$ Take and wait for someone

$\checkmark \quad$ Drop someone off

$\checkmark \quad$ Change mode of transportation (go to train station, bus stop)

7. Meals

$\checkmark \quad$ Go out to eat (restaurant, fast food)

$\checkmark \quad$ To go (fast food, coffee, restaurant takeout)

8. Return home

9. Other

The user is then given the option to select a mode of transportation they used to complete their trip. Vehicle occupancy and driver versus passenger data are also recorded via user input. Figure 27 illustrates the menu of transportation modes a participant would select from on his mobile phone.

GPS data automatically gathered by the mobile phone is associated with information entered by the user, then sent and stored on a server for analysis. 


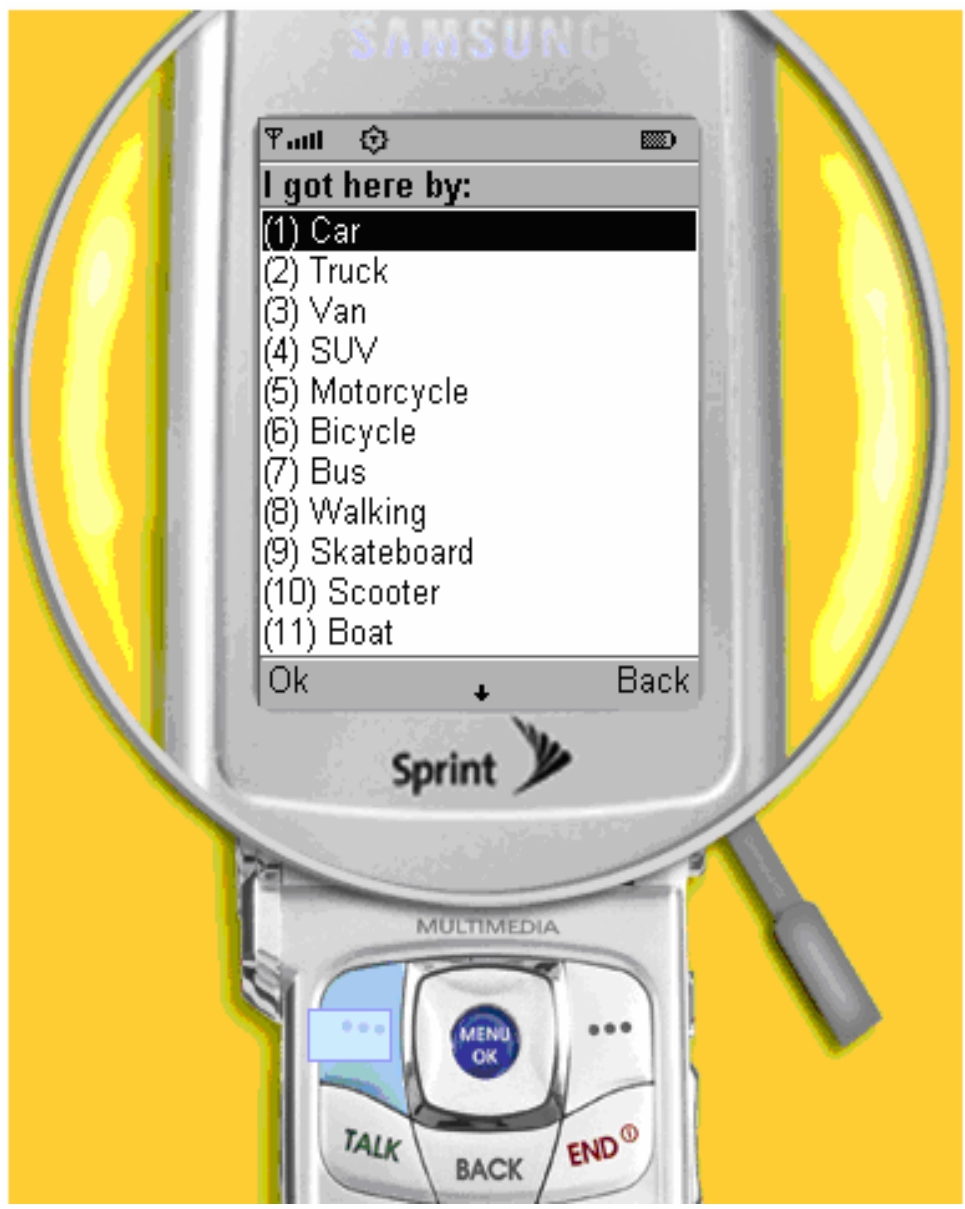

Figure 27 - Menu of Transportation Modes for Selection by Participant

\subsection{Data Validation and Suggestion Delivery}

The Expert System component of the Personalized Travel Coach automatically analyzes and generates suggestions for trips recorded using TRAC-IT. Although instantly generated at the server, in the current TRAC-IT system these suggestions, along with a visual representation of related trip information, are not autonomously delivered from the expert system to participants. For TRAC-IT Phase 2, Microsoft Outlook calendar was used to manually send a description of daily trips including a map image for easy visual validation with any suggestions generated by the Expert System if applicable. Trip information was sent in the form of a meeting invitation, as shown in Figure 28. It was assumed that if the meeting invitation was not rejected, the trip information was valid. An advantage of using a calendar such as Microsoft Outlook is that it allows the participant a visual tool to appreciate how time devoted to travel fits his daily schedule. 


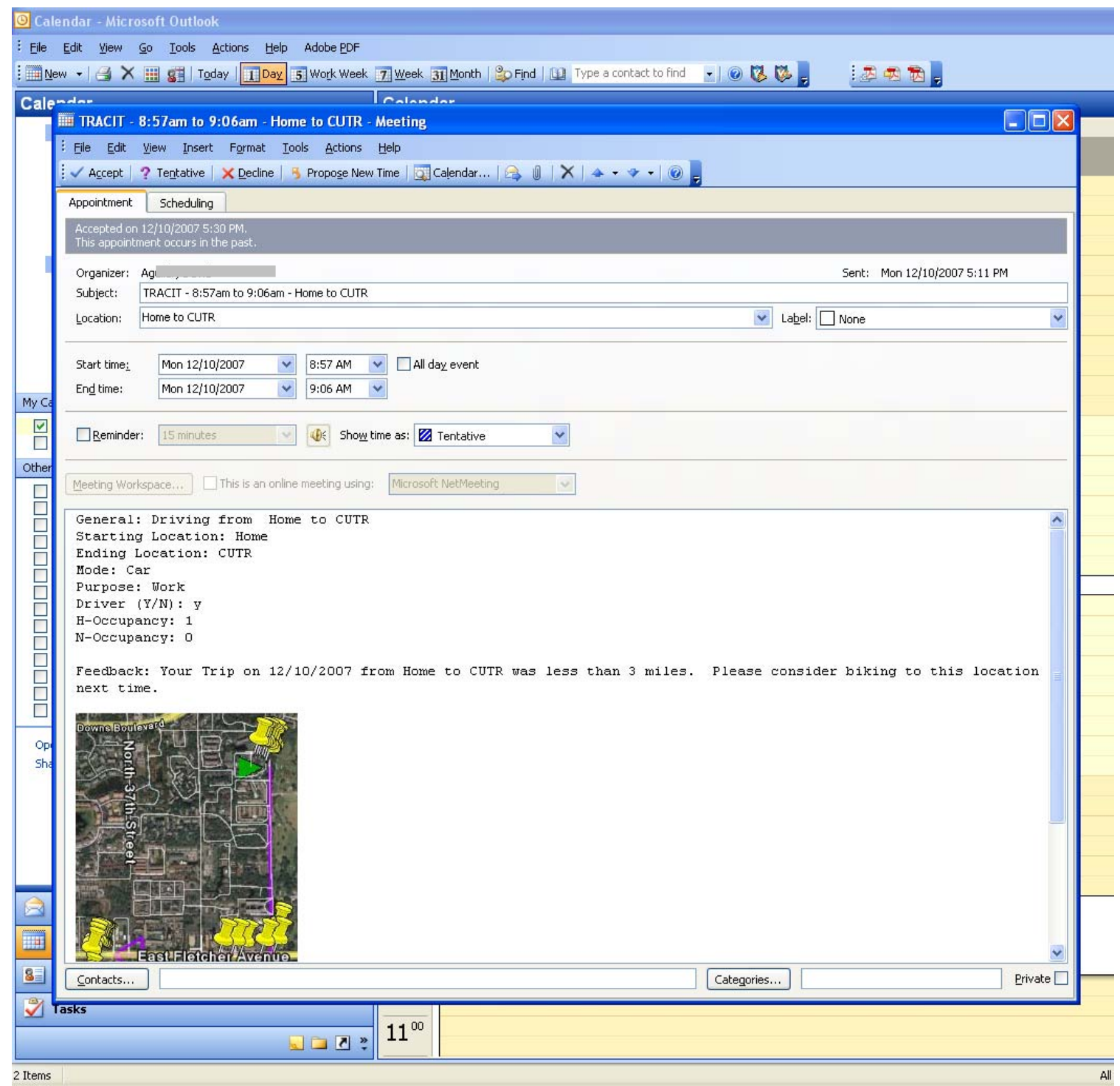

Figure 28 - Trip I nformation and Feedback as an Outlook Meeting I nvitation

These features are invaluable when encouraging travel behavior changes such as trip chaining, since participants view their trips made over an extended time period.

Not only does Outlook give the participant a validation tool for trips recorded by his mobile phone, but also the opportunity of sending their comments to the research team about the travel suggestions sent to them. For example, the participant can reply to the email informing the team that the suggestion of biking or walking to the store was not applicable for them because of health reasons or lack of sidewalks. In the future, Outlook may provide opportunities for enhancing TRAC-IT as a survey tool allowing participants to add trips missed while recording with the GPS-enabled mobile phone due to human or technical errors but could be added to the survey by clicking on calendar schedule. 


\subsection{Recruitment and Deployment}

This section details the recruitment and deployment associated with the TRAC-IT phone software application in field testing.

\subsubsection{Deployment Analysis}

For TRAC-IT to run on a mobile phone, an unlimited data plan from the wireless carrier is required. TRAC-IT also must be supported by the following technical phone features:

- Java-Compatible phone

- Support for JSR179 Location API 1.0 or JSR293 Location API 2.0

- Multi-tasking Virtual Machine (MVM)

- Supports User Datagram Protocol (UDP) and Hypertext Transfer Protocol (HTTP) data transmissions

As of June 1, 2007, all Nextel Integrated Digital Enhanced Network (iDEN) phones are supported, and all phones released after the Sanyo 7050 and the Samsung Upstage are supported on the Sprint Code Division Multiple Access (CDMA) network. The two necessary features that limit legacy Sprint phones from being TRAC-IT compatible are the lack of support of JSR179 and lack of MVM.

Digital signing is a process by which an identifier that directly links an application to a particular identifiable party has been independently verified by another trusted source. Since TRAC-IT must access sensitive APIs such as, for example, user information and GPS location of the phone, the application must be signed before installing it on any phone. Digital signatures are used by cellular carriers to ensure that only trusted or signed applications can be installed on mobile phones:

- For Nextel iDEN phones, Sprint-Nextel must sign the final TRAC-IT application so it does not expire after 48 hours. Once signed, the application cannot be modified without being re-signed.

- For Sprint CDMA phones, there are two options:

$\checkmark \quad$ The TRAC-IT application can be signed with a Verisign Secure Socket Layer (SSL) certificate so the research team can modify and re-sign whenever necessary to fix software issues. Up to 200 phones can be registered and tested for development purposes under the SSL certificate.

$\checkmark \quad$ Sprint can sign the application when finalized. This option does not allow the flexibility required for the creative iterative process software development undergoes to be deployable. Iterations of modifying, testing, tweaking, and verifying the application will have to stop before Sprint signs and allows TRAC-IT to run on any device. 
For future wide-scale deployments (more than 200 phones) this option would be the most appropriate.

The following potential scenarios detail the options for including participants in the TRAC-IT testing, ranked by the most to least likely scenarios that the research team would encounter during deployment as of 6/1/2007.

\section{Nextel Options}

- A Nextel user with a TRAC-IT-compatible phone and without unlimited data plan:

$\checkmark \quad$ Cell phone plan will have to be updated to include an unlimited data feature at a cost of $\$ 10$ per month.

$\checkmark \quad$ TRAC-IT can be installed on their phone through a cable hookup to a CUTR laptop or through a website. Sprint-Nextel would have to enable the deployment.

- A Nextel user with a TRAC-IT-compatible phone and with unlimited data plan:

$\checkmark \quad$ TRAC-IT can be installed on their phone either through a cable hookup to a laptop or through a Sprint-Nextel website. Sprint-Nextel would have to enable the deployment

\section{Sprint Options}

- A Sprint user without a TRAC-IT compatible phone and without an unlimited data plan

$\checkmark \quad$ There are two options to give them a compatible phone:

Forward their calls from their phone number to a CUTR Sprint phone with unlimited plan. The cost would be $\$ 0.20$ per minute rounded up to the nearest minute for forwarded calls, which may be cost-prohibitive for Sprint plans.

- Buy a Sprint CDMA phone (i.e. Sanyo 7050 for \$60) and temporarily replace their phone with the Sanyo 7050 phone, plus pay to add an unlimited data plan to their account at $\$ 15$ per month.

$\checkmark \quad$ TRAC-IT can be installed to their phone by text-messaging a link to a USF website to the phone. The participant would download from that link to their phone.

- A Sprint user without a TRAC-IT compatible phone and with an unlimited data plan

$\checkmark \quad$ There are two options to give them a compatible phone:

Forward their calls from their phone number to a CUTR Sprint phone with unlimited plan. The cost would be $\$ 0.20$ per minute for forwarded calls which may be cost-prohibitive for Sprint plans and the TRAC-IT user would still use the cell phone for personal calls. 
Buy a Sprint CDMA phone (Sanyo 7050 is $\$ 60$ ) and temporarily replace theirs with a Sanyo 7050 phone.

$\checkmark \quad$ TRAC-IT can be installed on the participants phone by textmessaging a link to a USF website to the phone.

- A Sprint user with a TRAC-IT compatible phone and without an unlimited data plan

$\checkmark \quad$ The user plan can be upgraded to include an unlimited data plan for $\$ 15$ per month.

$\checkmark \quad$ TRAC-IT can be installed on the participants phone by textmessaging a link to a USF website to the phone.

- A Sprint user with a TRAC-IT compatible phone and with an unlimited data plan

$\checkmark \quad$ TRAC-IT can be installed on the participants phone by textmessaging a link to a USF website to the phone.

Ideal options are those with no additional incurred costs where the user's phone is TRAC-IT compatible and has an unlimited data plan. The research team anticipates that these two scenarios will become more common and widespread in late 2008 to early 2009.

\subsubsection{Recruitment Methods}

Since individuals must use particular wireless providers and have certain model mobile phones, efficient recruitment can be a difficult obstacle. The research team identified a promising new method of recruitment referred to as Wireless Application Protocol (WAP) advertising. WAP advertising is a version of online advertising for web pages appearing on mobile phones, referred to as WAP pages. A banner appears on the user's phone while the user is browsing the web or reviewing his or her account information, and the user can click on it to be directed to a page with further instructions or advertising. WAP advertising differs from traditional online advertising in that it is controlled by the wireless carrier, so a banner ad can be inserted by the carrier on any web page that is visited. Additionally, ads can be targeted to individuals in certain geographic areas, certain cell phone models, and plans with certain characteristics (i.e., family plans). Therefore, WAP advertising has the potential to reach the exact intended population, even down to which cell phone models are supported by the mobile TRAC-IT application. The team planned to use WAP advertising to recruit households for the TRAC-IT Phase 2 field tests.

Due to the current limitations of cellular carrier deployment of Location-Based Services technology (see TRAC-IT Phase 3 Final Report), as of June 2007 the Sprint-Nextel CDMA network (formerly Sprint PCS network) and Sprint-Nextel iDEN network (formerly Nextel iDEN network) are the only U.S. carriers that support the standards required by the TRAC-IT system. Therefore, customers from these two networks were targeted for the field tests once the system integration was 
deemed stable. WAP advertising would be used as the primary recruitment method.

For each Nextel customer, the cost would likely be $\$ 10$ per month per person to add unlimited data service to his or her plan. The existing phone could be used.

For each Sprint customer, the cost was $\$ 15$ per person per month to add unlimited data service to his or her plan. It is likely that the participant's phone will have to be swapped out with a TRAC-IT-compatible phone. A compatible phone, the Sanyo 7050, is $\$ 60$ and could be transferred from participant to participant during the field tests.

If the total number of participants is 60 (at most, 20 active at a time), and the estimated number of weeks per participant is 3 , and the survey sample is half Sprint users and half Nextel users, the estimated total cost of deployment was $\$ 1,350$.

\subsection{Survey Protocol}

When households were identified, a letter of solicitation that explains the research project and the survey involvement of the participant were provided to each household (included in Appendix). If the household was interested in participating, an informed consent document would be provided to each participating individual in the household (included in Appendix). Once the informed consent document was signed, the individual would be given access to an online orientation, discussed in next section. Once the individual completed the orientation, he would either be provided a TRAC-IT phone if his phone was not TRAC-IT compatible or would be instructed to download the TRAC-IT application to their TRAC-IT compatible phone.

\subsubsection{TRAC-IT Orientation Session: The Movie}

As part of this research effort, a new tool was developed to make the orientation session as convenient as possible for participants. The TRAC-IT power point presentation that was developed for live orientation sessions with participants as attendees was documented as a movie and put on the NCTR website online at http://www.nctr.usf.edu/flash/TRACIT\%200RIENTATION\%20SESSION.htm.

A selection of screen shots from online orientation session is included in report Appendix. Figure 29 is a screenshot of the first slide. The audio played with the slide was recorded by one of the research team members and reads:

Welcome to the orientation session. Thank you for being a part of this research effort. Your willingness to participate is greatly appreciated. This session runs like a movie, please feel free to play, pause, forward or rewind the movie. 


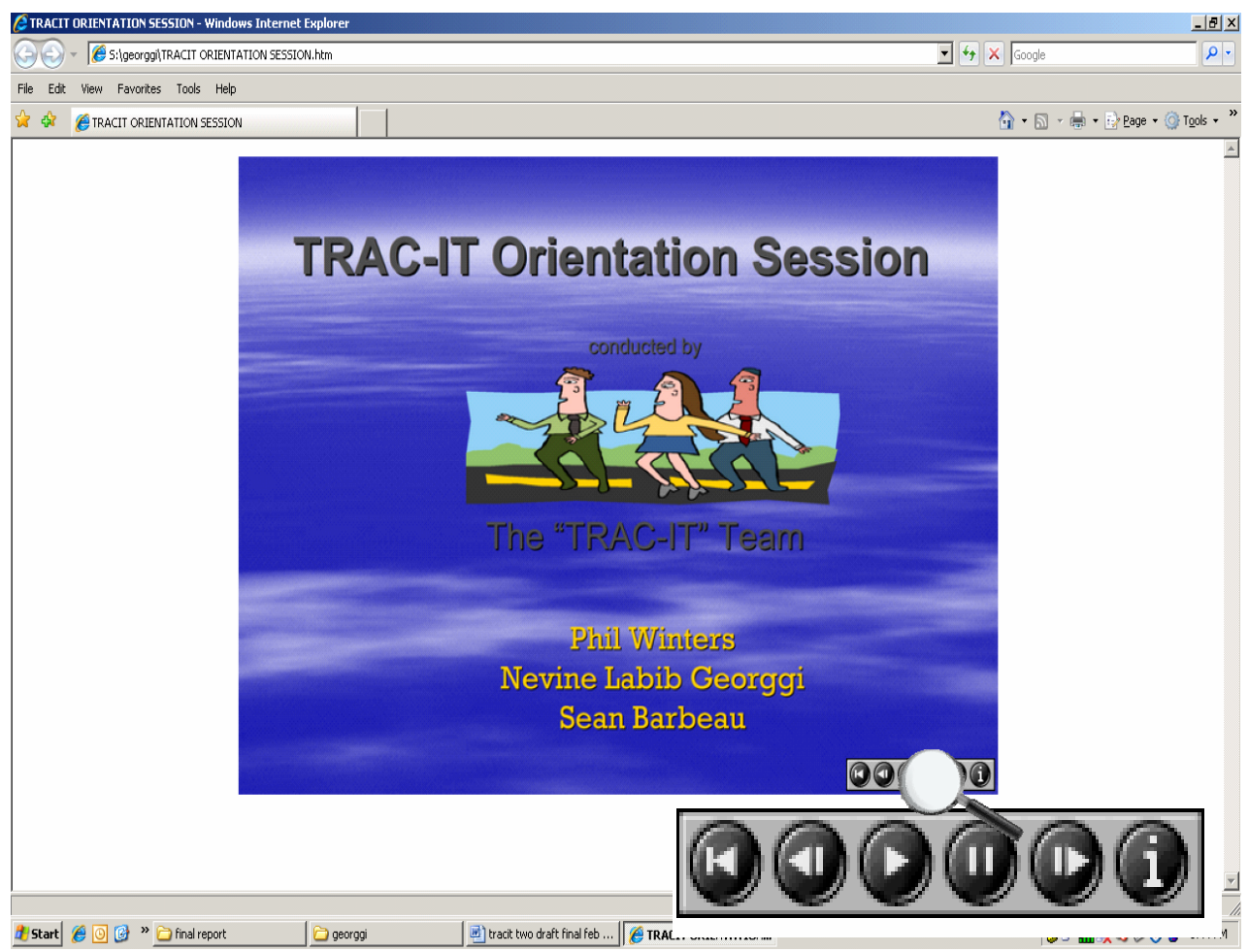

Figure 29 - TRAC-IT Orientation Session Played as a Movie

The movie continues with the audio explaining the goals and objectives of travel surveys and the role the participant will play in making the research beneficial to the community covering the following outline:

- What is a travel survey?

- What is it used for?

- Why collect the data?

- Who is conducting the survey?

- Who is participating?

- Where are the data collected?

- When are the data collected?

- How are data collected?

- How is my privacy protected?

- Trip - how is a trip defined?

The following are screenshots of the consecutive slides that are included in the first portion of the movie. The actual slides follow the slide design shown in Figure 29 but are displayed in black and white in Table 4 for clarity and legibility. 
Table 4 - Sample Screenshots of the Flash Animations of TRAC-IT

TRAC-IT Orientation Session

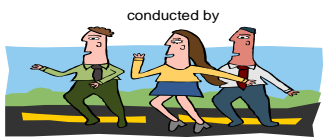

The "TRAC-IT" Team

Phil Winters Nevine Labib Georggi

Sean Barbeau

\section{We need you \\ to participate in a daily \\ travel survey,}

\section{using a GPS-enabled} cell phone.

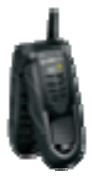

Slide 2

Slide 1

What is a Travel Survey?

What, Why, Who ... Travel Surveys

- What is a travel survey?

$$
\text { - What is it used for? }
$$

- Why collect the data?

- Who is conducting the survey? - Who is participating?

- Where are the data collected?

- When are the data collected?

- How are data collected?

- How is my privacy protected?

- $\quad$ Trip - how is a trip defined?

Slide 3

Why Conduct a Travel Survey?

- Transportation infrastructure improvements are very expensive and require extensive planning in advance.

- Data from survey is used to predict future demand on area roads, trains, and buses (at least 20 years into the future).

- If we know where and what we need to build, then we have a better chance of making timely, cost- effective improvements.
- A tool to collect data about daily journeys of individuals or households including information about:

- an individual (socio-economic, demographic, etc.),

- their household (size, structure, relationships),

- their vehicles if any (age, make, model), and

- a diary of their journeys on a given day (their start and end time and location, mode of travel, purpose of trip, number of household members making the trip).

Slide 4

Who is conducting the survey?

- The National Center for Transit Research at the Center for urban Transportation Research at the University of South Florida

- Person in Charge

Philip L. Winters

TDM Program Director

Center for Urban Transportation

Research

University of South Florida

4202 E. Fowler Ave., CUT100

Tampa, FL 33620-5375

(813) 974-9811

winters@cutr.usf.edu 


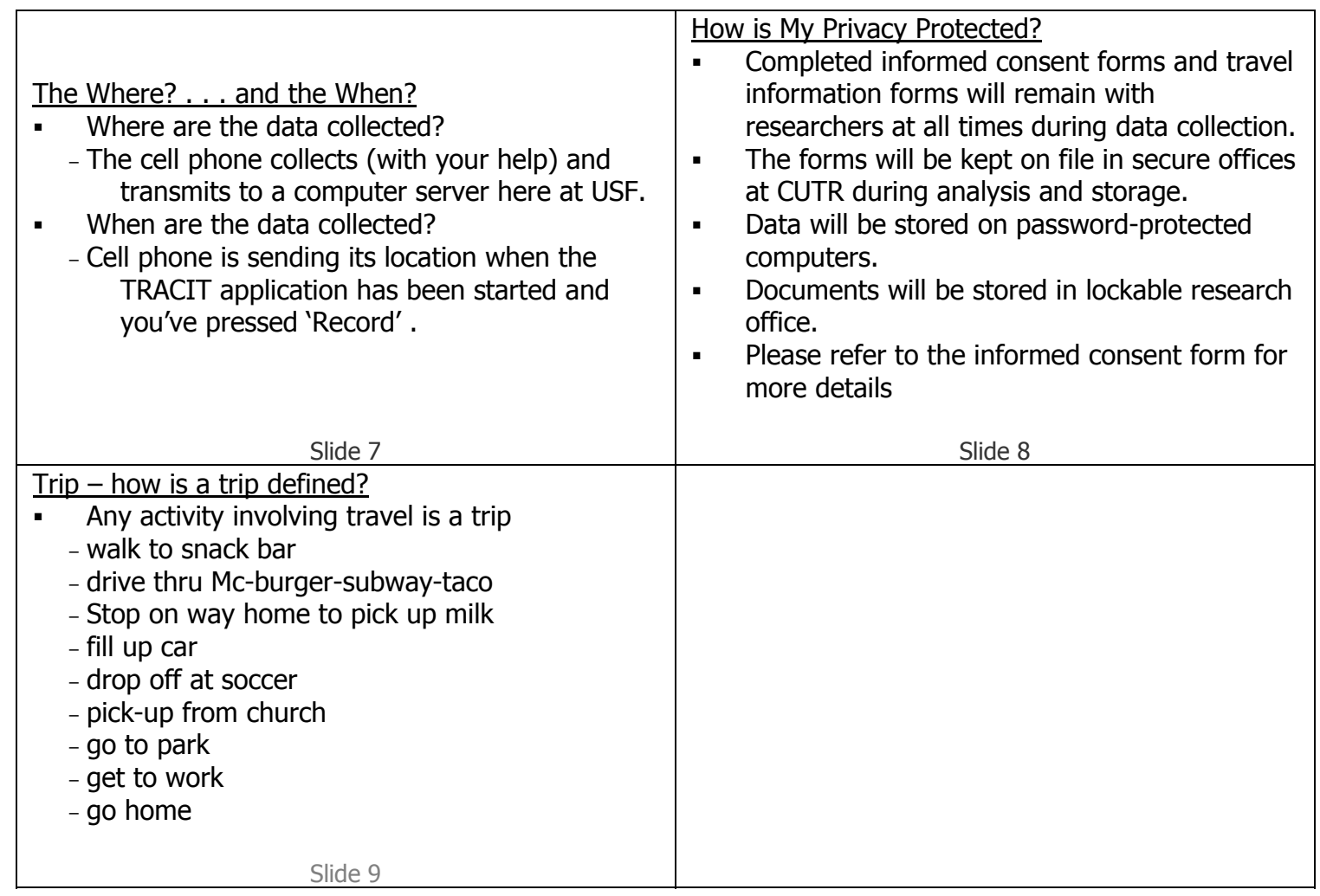

The narrator of the movie then introduces the Demo portion of the session. The Demo is a click-by-click animated Flash video of a simulated mobile phone with TRAC-IT application running. Each animation takes an average of 3-5 seconds to display how the TRAC-IT application works and how Quickstops can be used.. Approximately 50 sequential animations are shown to the user who can still pause or play the movie at their own pace. Figure 30 is a collection of screenshots from the TRAC-IT mobile application being executed on a mobile phone emulator.

After the demo is played, the narrator gives a few reminders and pointers to the participant using with power point slides. If played at its own pace, the entire session should be run in about 30 minutes.

This electronic orientation session can be uploaded to the Internet, emailed as an attachment, or sent to a survey participant in a CD-ROM format. The advantage of the electronic session is that survey participants can view it at their own convenience and their own pace. Also, they can repeatedly view it in case they need to refresh their memory on how to use TRAC-IT. If consent forms were to be electronically signed, the entire recruitment process of participants can be remotely conducted eliminating the time it would take both survey participants and researchers to conduct and attend live orientation sessions. 


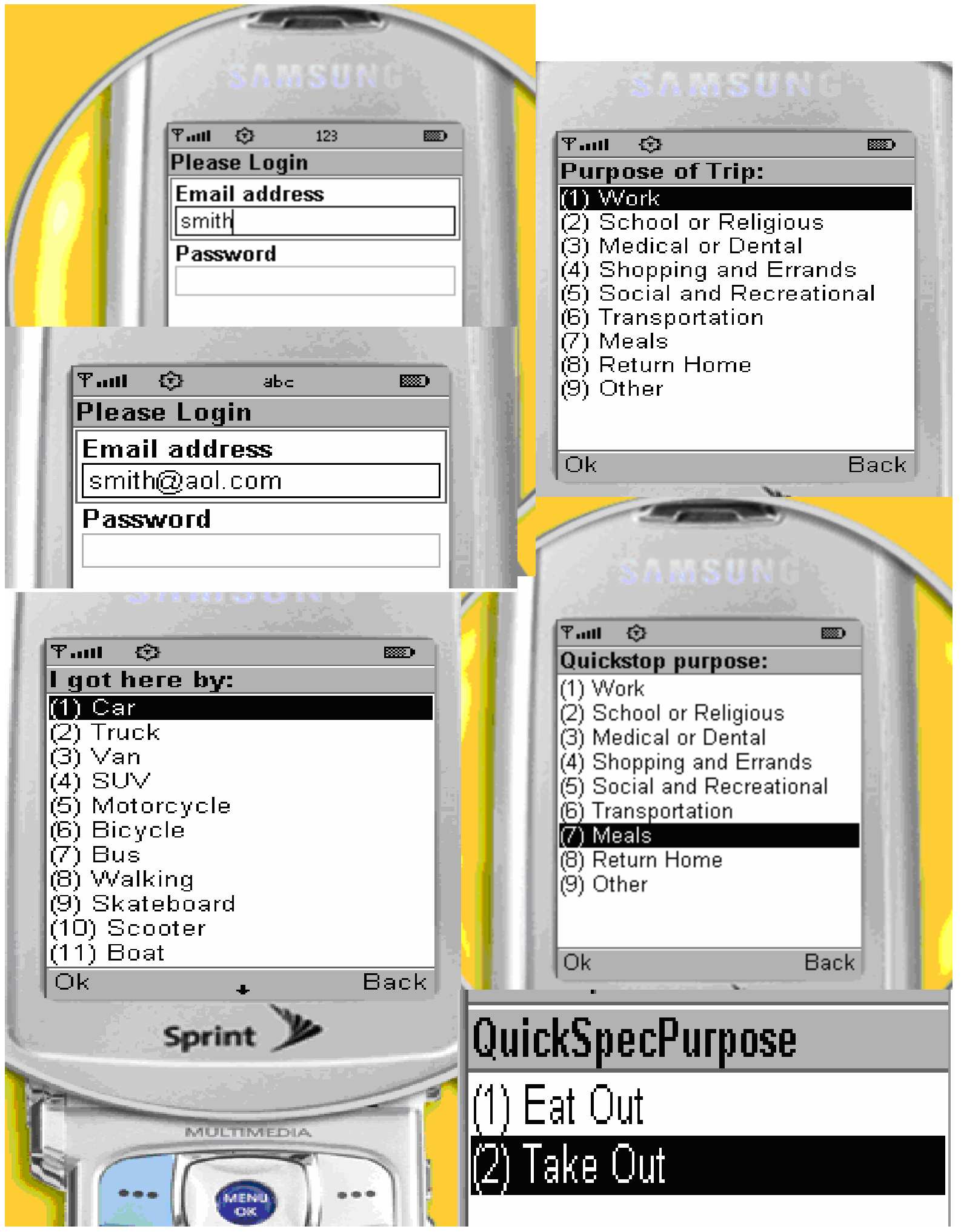

Figure 30 - A Collection of Screenshots from TRAC-IT Mobile Application 


\section{Chapter Six - Survey Analysis and Findings}

The TRAC-IT Phase 3 field tests served as an initial proof-of-concept evaluation of the cell phone's ability to gather transportation behavior data. The same tests were used for evaluation of the Expert System Component of the Personal Travel Coach. These tests successfully demonstrated the TRAC-IT mobile phone application's potential of serving as a next-generation travel survey tool. However, issues related to the integrity of recorded trip data were identified and further research is recommended to fine tune the application and improves system reliability. A detailed documentation is included in the TRAC-IT Phase 3 final report. Issues detected are summarized in the next sections.

For Phase 2, it was determined that travel behavior changes, analyzed by comparing experimental and control groups before and after receiving personal travel coaching, would be invalidated if based on patterns not accurately representing household travel. Due to complications related to new policy-based cell phone usage restrictions by some state agencies and challenges with leapfrog technologies this study pursued, the research team decided to limit the field testing task of this phase to the tests conducted for TRAC-IT Phase 3 with the analysis of feedback generated by the Expert System focusing primarily on trips recorded by participants of the Phase 3 survey.

\subsection{Initial Testing of Survey Instrument}

Initial testing of the TRAC-IT system was done by the research team members and their households. While trip information was gathered during this testing, a number of issues were identified, including critical technical issues that caused loss of trip data. A summary of issues identified in this leapfrog technology project and steps taken to resolve them when possible are discussed next.

\subsubsection{Policy-Based Restrictions}

During the research performed for this project, USF changed internal policies related to purchasing cell phones and service plans under research grants. While the policy was designed to prevent the abuse of personal expenses being charged to grants, it also unexpectedly impacted research projects. As a result, an exception request process and corresponding approval were required for each USF grant before any cell phones or service plans could be purchased. This process took nearly eight months before university officials approved the exception request. This unanticipated delay impacted the duration of field testing possible under TRAC-IT Phase 2 and Phase 3 as well as research conducted by graduate students hired for this project.

Gaining access to test TRAC-IT on the cellular networks was also more challenging than expected. Although GPS technology that would support TRAC-IT is 
embedded in many phones, most cellular carriers impose strict access restrictions due to privacy concerns related to location-aware applications. While the research team approached all major cellular carriers and requested access to test TRAC-IT, only the Sprint-Nextel Developer Program granted access to test TRAC-IT on its CDMA network and iDEN networks. The project managers acknowledge and appreciate Sprint-Nextel for encouraging the cutting edge nature of this research series.

\subsubsection{Rapidly Progressing Technology}

Mobile phone technology progresses at a very rapid pace. To address new technology needs, research projects must adapt accordingly. Among the most significant challenges encountered during the project were the merger of Sprint and Nextel into Sprint-Nextel and the emergence of next-generation high sensitivity GPS-enabled mobile phones on the Sprint-Nextel CDMA network. New technology creates new opportunities such as improved GPS reception and network-based positioning when GPS is not available. However, it creates new challenges for TRAC-IT as it must be reconfigured to take advantage of these new features.

\subsubsection{Blocking I ncoming Phone Calls}

One problem discovered with the initial implementation of TRAC-IT was that frequent data transmissions (e.g., every 4 seconds) blocked the reception of incoming phone calls. As a result, phone calls would go to voicemail. TRAC-IT has since been modified to avoid occupying the transmission channel when it is not necessary to do so. One improvement in this area is the ability to selectively buffer data on the cell phone. The feature collects several location fixes until the defined buffer limit is reached and then transmits all buffered data at once to the server. This method occupies the communications channel for less time than transmitting location data every time a new fix is obtained.

\subsubsection{Limited Battery Life}

Battery power was reported to be as short as four hours when TRAC-IT was used in the passive recording mode. Active recording (i.e., TRAC-IT only records when the user manually starts trip recording) had significantly improved battery results. Several new power-saving features have been added to the TRAC-IT cell phone application that attempt to improve general performance and to make passive tracking feasible. One algorithm lowers the polling frequency of the GPS system when indoors and/or when a GPS signal cannot be found but then increases the polling rate when the phone regains a GPS signal to ensure a detailed path was still recording. Another algorithm filters location data on the mobile phone and transmits only critical points required to reconstruct the user's route to the server. Further testing of these algorithms is required to ensure the improvement of 
software stability and to optimize the settings in the algorithms to maximize battery life. User settings have also been developed to allow the user to switch these algorithms on and off.

\subsubsection{Privacy Concerns for Passive Tracking Feature}

In preliminary tests, users requested the ability to turn passive tracking on and off in order to better protect their privacy. User settings now allow participants to control exactly when their travel behavior is being recorded while still allowing TRAC-IT to run in the background at all times. Users can turn passive tracking on and off via the Settings menu to allow them to opt out of being tracked if passive tracking is being used.

\subsubsection{Users Forgetting to Record Trips}

One of the top issues that all participants reported was the difficulty of remembering to start recording their trip when using TRAC-IT in active recording mode. However, the participants reported that they remembered to carry the phone with them. A new feature was added that reminds users to record their trips using TRAC-IT when they walk outside a building. The TRAC-IT mobile phone application now beeps when a first GPS fix is obtained after a long period of no GPS signal detection. Future development work on this method is required to adapt it to high-sensitivity GPS devices that can get occasional GPS fixes indoors.

\subsubsection{Server Stability}

Server software stability is an ongoing issue due to multiple potential points of failure in the system and lack of redundancy (e.g., back-up server). Within the TRAC-IT system there are several software applications that function as a whole to be the TRAC-IT server. The main applications that handle real-time server functionality are Glassfish, a Java application server that hosts the web services with which the TRAC-IT mobile phone software interacts, and SQL Server 2005 database server. Other system components such as the Expert System are launched separately to process information once it is collected in the database. All of these applications also include subcomponents, such as the connection pool and Java Database Connectivity (JDBC) drivers for the Glassfish Java application server. All of these software instances are being continuously reconfigured to provide greater stability for the server and to address issues as they occur. New configuration features that have been added include the automatic restart of the Glassfish if the server computer is rebooted. Separate Glassfish server software instances have also been established for each server application, including TRACIT, to limited interference between them. Glassfish has been upgraded to the newest version, and the JDBC drivers have been updated to the newest versions for Microsoft SQL Server. Glassfish database connection pools have been 
continuously reconfigured to prevent connection leaks and to provide optimal functionality. Microsoft SQL Server 2005 has been updated to the newest service packs and has been continuously reconfigured to improve system stability. Two hardware servers have also been established so the research team can continue to troubleshoot issues on a development server while the current deployment of TRAC-IT remains unaffected on a production server. However, issues related to the database table locking when multiple concurrent trips are being recorded repeatedly occurred, causing loss of trip data. Additionally, when these issues occur, the server subsequently locks users out of the system until the symptoms are resolved through manual intervention by a system administrator. These lockups result in an unknown number of trips being lost while the server is down. For further information on the TRAC-IT system architecture, please refer to the TRAC-IT Phase 3 final report.

\subsubsection{TRAC-IT Mobile Phone Software I ssues}

When the phone saves information locally, it was found that occasionally data can become corrupted. Work has been done to help avoid this problem by retrieving information from the server to replace the corrupted information. Also, program redundancy enhancements were made so that when the TRAC-IT application shuts down, all data is written to persistent storage correctly. Other issues related to intermittent server connectivity seem to continuously occur for certain users, causing locations not to appear in the TRAC-IT mobile phone user interface.

Another connectivity issue occurs if the user is outside of data coverage when the trip either begins or ends, resulting in the phone not being able to properly record the entire trip. This issue results in partial trip records being registered with missing end information, or the trip is not registered at all. Intermittent connectivity issues are difficult to troubleshoot since the problem may lie in the phone hardware, phone firmware (i.e., operating system), the TRAC-IT phone application, cellular network coverage and/or roaming on other networks, the Internet, the CUTR network, the TRAC-IT Glassfish server, the TRAC-IT server operating system, or a combination of any of these areas. Since the TRAC-IT mobile phone application relies on information from the server, problems with phone software could actually originate from problems with the server. Before these issues are completely resolved, extensive troubleshooting will be required and new features implemented, such as caching trip data on the mobile phone during service interruptions.

\subsection{Expert System Feedback from TRAC-IT 3 Field Tests}

The following sections discuss the results of the Expert System Feedback component from TRAC-IT Phase 3 field testing and a brief analysis of the results. 
Fourteen volunteers were individually solicited to participate in the TRAC-IT Phase 3 field testing, which lasted 2 to 3 weeks per participant. A number of mobile phones, including models Sanyo SCP-7050 on the Sprint-Nextel CDMA network and Motorola iDEN i580 on the Sprint-Nextel iDEN network, were distributed to the 14 volunteers. The location of the user's activity was primarily on and around the USF campus area in Tampa, although some trips were recorded in Seattle, Fort Lauderdale, and Orlando to test TRAC-IT in other geographic locations. A variety of modes were utilized, including walking and riding transit with vehicle trips being the most common.

During field testing, 317 trips were recorded in the remote database by the 14 volunteers spread out over a period of three months, resulting in the collection of 66,523 location data points. In 32 cases (approximately $10 \%$ of trips), the end trip information recorded by the participant using the user interface of the phone was lost and not recorded in the database due to technical issues with the TRACIT system. An unknown number of trips were not recorded due to technical issues, which intermittently prevented the use of the TRAC-IT system.

Of the 317 trip segments recorded in the field testing period, 160 produced feedback advice from the Personal Travel Coach Expert System. The remaining 157 trips were determined as acceptable by the Expert System and no feedback was generated for these trips. The breakdown of travel advice by type is shown in Table 4:

Table 5 - Breakdown of Personal Travel Coach Feedback by Type

\begin{tabular}{|c|c|c|}
\hline Advice Type & $\begin{array}{c}\text { Number of } \\
\text { times offered }\end{array}$ & $\begin{array}{c}\text { Rate of } \\
\text { advice type }\end{array}$ \\
\hline $\begin{array}{l}\text { To chain trips (travel for more than one purpose on the } \\
\text { same trip) }\end{array}$ & 39 & $24.4 \%$ \\
\hline To walk & 37 & $23.1 \%$ \\
\hline To bike & 33 & $20.6 \%$ \\
\hline To carpool & 20 & $12.5 \%$ \\
\hline To shop on-line & 15 & $9.4 \%$ \\
\hline To combine trips & 12 & $7.5 \%$ \\
\hline To alter travel schedule to avoid rush hour & 4 & $2.5 \%$ \\
\hline Total & 160 & $100 \%$ \\
\hline
\end{tabular}

Research team members that participated in the survey determined that almost all feedback generated for their trips was appropriate, with the exception of several cases where the advice returned was impractical, mostly due to user error. For example, several trips to schools by users to drop off children were mistakenly labeled with the purpose of School->Go to School instead of Transportation>Drop off Person, and therefore suggestions were made to consider biking or walking to the location. Future versions of TRAC-IT should consider enhancements to prevent this type of user error. 
Some suspect feedback was the result of TRAC-IT system errors. For example, a software error in distance calculation resulted in some trips having inaccurately long distances, which triggered shop on-line suggestions when they were not appropriate since the actual traveled distance by the participant was much shorter than the calculated distance.

There are still other cases where further modification of the rules is required to add certain preconditions that would prevent suggestions being given in particular scenarios. For example, the following feedback was given to one user: "Your trip on 12/6/2007 from GAS STATION to Publix was less than 1 mile. Please consider walking or biking to this location next time." To prevent this feedback, another precondition should be added to the distance-based rule that states if the trip being examined is a segment of a trip chain (i.e., previous trip was a quick stop made on the way to another trip) and the purpose was, for example, Car Services, then the walking or biking advice should not be generated. 


\section{Chapter Seven - Conclusions and Recommendations}

The TRAC-IT system is a leapfrog technology research project that should advance the quality and quantity of household multimodal data collected in travel surveys. Using the TRAC-IT application for both Personal Travel Coach components, the Expert System, and the Path Prediction Prototype, gives travel surveyors the next-generation cutting edge they need to provide transportation professionals and decision-makers with the data needed to understand and plan for consumers' needs. Inherent with such innovative projects are unforeseen challenges, both technical and policy-based.

Technical integration hurdles are associated with location-aware information systems when combined with mobile phones, overlapping software, wireless carriers, and multiple server-side infrastructures. Rapid technological advancements in the telecommunications industry, including the merger of major carriers (Sprint and Nextel) and the emergence of next-generation high-sensitivity GPS-enabled mobile phones on the Sprint CDMA network, created new opportunities with new challenges. Unanticipated and interruptive policy changes such as USF's restriction on cell phone purchases regardless of their proposed use delayed development and testing TRAC-IT for months. Other challenges such as the WAP advertising issue, previously discussed in this report, hindered the project progress.

The combined impacts of these factors resulted in creation of a potentially revolutionary but not thoroughly vetted travel behavior data collection and analysis system. System instability issues uncovered during TRAC-IT Phase 3 testing resulted in the research team's decision to continue developing and refining TRAC-IT as a data collection tool rather than attempting to measure travel behavior changes of test and control groups in a before and after survey approach as originally planned as one of TRAC-IT Phase 2 objectives. At various stages in the development and deployment of TRAC-IT Phase 2 and Phase 3, it appeared that field testing with more households would be possible. New technical issues continued to emerge causing loss of data that would have invalidated any analysis of travel patterns collected using the system.

Intermittent interruptions in cell phone/server connections due to hardware and software configurations supporting the TRAC-IT system, such as security updates requiring rebooting the server, prevented users from reliably recording all their trips. Similarly, issues in TRAC-IT software prevented users from completing records of all their trips, including how the mobile application could not always show a list of saved locations to user causing further issues with software wizards and locking the database from access by other phones. Not only were trips not complete, but the research team observed a decline in reported trips when the system was operational. Field test participants expressed their frustration about 
the inability to log into their cell phone to record new trips on a consistent basis. This contributed to a loss of an unknown number of trips not recorded by participants in addition to the known 10 percent loss of end trip data because of technical TRAC-IT problems. Clearly, the need for redundant servers and a robust error handling system would be essential for large-scale implementation.

The loss of trip data is not a trivial matter when automated analyses by intelligent systems are being performed. The Expert System depends on a comprehensive record of the user's travel behavior to provide meaningful feedback. When comprehensive data were available, the system provided precise and insightful suggestions. Losing data would underestimate the number of trips taken by participants, resulting in incomplete feedback that should have been given to the experimental group, rendering inconclusive measurements of travel behavior changes of that group versus the control group. The decision was made to focus the research resources on increasing the reliability of the system rather than purchasing additional phones for a large-scale survey using TRAC-IT while the system was still experiencing intermittent technical problems. Though the testing of the effectiveness of feedback advice using a before and after survey was not accomplished, this leapfrog research project has made the following significant contributions:

1. The development of an enhanced Personal Travel Coach with a new and improved implementation that supports eight rules that analyze household and individual travel patterns before providing suggestions.

2. The redesign of the TRAC-IT travel data collection tool to include userfriendly wizards with minimal input from the participant. The PDA version was successfully transferred to a new cell phone version. The new software system (i.e., server and mobile phone software) that supports GPS-enabled mobile phones was developed and tested. This mobile phone version is the first software-only travel behavior data collection tool that can track a person's travel behavior regardless of mode (e.g., auto, bus, bike, and walk) by using a hardware device that most people already own and is always with them as opposed to previous systems that track vehicle (e.g., AVL for transit, Vehicle Navigation Systems for auto) or require proprietary devices dedicated to data collection.

3. It increased the depth and breadth of travel behavior data with minimal burden on the participant by automatically collecting route, travel time, time and day of week, and travel distance from GPS data while still collecting survey data input manually from the user for items such as purpose, mode of transportation, beginning and ending location descriptions, and occupancy.

4. It identified how to passively collect trip purpose through integration of GPS position data with local tax GIS records. 
5. It identified how to passively collect mode of transportation data through a mode detection algorithm.

6. It introduced feedback in the form of email with a visual map to reach participants after they have finished recording their trip so participants can instantly confirm their trip details via a reply email before they forget.

7. It developed the Path Prediction prototype module of the Personal Travel Coach that is able to predict the users' travel paths based on their real-time location and their previous personalized travel behavior history. Path prediction allows the TRAC-IT system to detect potential incidents that lie within the user's possible travel route and push travel advice to the user in real-time via text or audio alerts to the mobile phone before they reach problem areas. This TRAC-IT feature should increase the effectiveness of 511 information systems towards changing travel behavior by moving to an information push model instead of pull (i.e., user automatically receives notices instead of calling into the system to retrieve data) while assuring only relevant information is sent to the user (i.e., location information is used to filter out irrelevant data).

8. It developed an electronic orientation session that can be uploaded to the Internet, emailed as an attachment, or sent to a survey participant in a CDROM format. The advantage of the electronic session is that the survey participant can view it at their own convenience and their own pace. Also, they can repeatedly view it in case they need to refresh their memory on how to use TRAC-IT. If consent forms are electronically signed, the entire recruitment process of participants can be remotely conducted eliminating the time it would take both survey participants and researchers to conduct and attend live orientation sessions.

Once the TRAC-IT system is stabilized, the experimental testing outlined as prepared for this project should be carried out in the future as the potential benefits of impacting travel behavior by providing personalized feedback have proven effective in previous pilot studies. The following tasks must be accomplished before TRAC-IT is deployed and the experimental testing is conducted:

(1) Resolve remaining TRAC-IT technical software issues:

- Distance calculation - Software that handles distance calculation is an issue that occasionally results in erroneously large distance measurements. This problem should be resolved so distance-based expert system rules can provide more accurate advice.

- Database table locking - There is a problem with the TRAC-IT system during periods of intermittent connectivity between the mobile phone and server. If there is a communication failure (i.e., cellular network not available) during times when the TRAC-IT user tries to begin or end a 
trip, the database is left in a state of an exclusive lock for the last phone that attempted access. Once this lock occurs, no other phones can perform any functions, including log-in to the system and starting or stopping trip recording until a system administrator manually intervenes. This issue must be resolved to stabilize the system and always allow users to log in and record their trips.

- Server restarts - There exists a configuration issue that requires the TRAC-IT server to restart when new updates become available to protect the system. However, this update process interferes with the TRAC-IT software server and does not always allow the system to restart properly. This is one of the reasons users are occasionally prevented from logging in and recording trips. The server must be configured to schedule restarts at opportune times enabling the TRAC-IT software server to remain functional throughout this procedure.

(2) Implement enhanced TRAC-IT features to resolve existing weaknesses and improve system performance:

- Trip data caching - The current version of TRAC-IT relies on always-on network connectivity in order to immediately transfer the results of a trip to the TRAC-IT server. This system design allows even low-end cell phones to use TRAC-IT since no on-board data storage for trips are required. However, there are many cases where data connectivity is lost to mobile applications due to lack of cellular coverage, roaming, or wireless interference. These issues account for the greatest loss in reported trip data for the current TRAC-IT system, necessitating an improvement to the system. To enhance the reliability of the TRAC-IT system, temporary on-board caching of trip data should be implemented in future versions. Temporary caching of trip data would allow TRAC-IT to remain operable even when network connectivity is interrupted and therefore is recommended as a critical module to be developed.

Although additional new features may not be critical to the implementation of experimental testing, it would greatly enhance the performance of the TRAC-IT system in general. Passive tracking is a feature that will optimize the number of reported trips by TRAC-IT and increase the validity of results. The number one usability issue reported by participants in the TRAC-IT 3 field tests was the trouble remembering to start recording trips using the TRAC-IT interface. However, participants did report that they did not have nearly as much difficulty remembering to carry the TRAC-IT mobile phone with them. Since the goal of TRAC-IT is for the software application to be installed on personal mobile phones, it is very likely that future participants carrying TRAC-IT on their own phone will always have the phone with them. Passive tracking, which does not require the user to actively start and stop, recording trips, is a feature that will decrease the number of missed trips. 
There are two main challenges when utilizing passive tracking that must be addressed: impact on device resources, especially battery life, and the privacy of the individual. Since TRAC-IT can always be running in passive data collection mode, it is vital that the application be highly optimized in order to prevent a negative impact on mobile phone resources. The application must not interfere with receiving phone calls and must also use as little energy as possible to prevent rendering the device useless by expending all battery power. Techniques developed for TRAC-IT, location data buffering, the state machine, and critical point algorithms should be further studied, and the benefits of these features should be quantified to better understand device performance that highly optimizes the TRAC-IT application.

By utilizing passive tracking, the TRAC-IT survey essentially is transformed from an opt-in model to an opt-out model. In other words, the current TRAC-IT model requires users to actively confirm that they want each trip they take to be recorded. Passive tracking would default to always recording a user's trip unless the user explicitly selects to prevent a trip from being recorded. Some participants may not be comfortable with this model, and therefore it is suggested that additional privacy protection measures be put in place when passive tracking is used. Such methods could include sensitive times or places to be defined by the user that would automatically prevent tracking during those times or near particular locations.

Other modules of the TRAC-IT system could also benefit from enhancements. Currently, while advice for participants is automatically generated by the Expert System, the methods of delivery are still manual. In the current version of the TRAC-IT system the feedback from the Expert System, as well related trip information, must be manually exported from the TRAC-IT database, formatted into an email (including the creation of a map screenshot showing trip route, origin, and destination), and then sent to the participant. Automation of this process would reduce the amount of labor necessary to provide feedback and visual trip information to participants in future TRAC-IT surveys. Additionally, this automation would allow the TRAC-IT research team to easily monitor the implications of different application settings and features designed to increase system performance during application testing. A variety of delivery methods other than email should also be evaluated, including the effectiveness of each method on impacting travel behavior.

Future research is needed for the Path Prediction algorithm. Most importantly, the algorithm should be implemented in real-time using GPS-enabled mobile phones, tested and analyzed with many more sets of trip data. The research team experienced many technical problems with the implementation of the algorithm in ESRI ArcServer and ArcObjects software. These problems prevented the execution of the algorithm in real-time using the TRAC-IT mobile phone and server software. Future implementations should explore alternative GIS systems 
that support spatial queries such as PostSQL with PostGIS or Oracle Spatial and determine if they offer more stability than the combination of ArcServer, ArcObjects, and ArcSDE with SQL Server 2005.

The Path Prediction algorithm can also be further improved to increase the accuracy of the algorithm by reducing false negatives and false positives as well as the time required to notify the user of incidents on their route. In future refinements of the algorithm, the stored buffers' widths could be increased via the Path Maker application to accommodate drifting GPS fixes that deviate significantly from the street network; this modification would improve the buffer detection when the formation of the polyline is not ideal. A more statistical approach for situations where no trip record exists for a given route could be used instead of the current method, but care should be taken to avoid increasing the computational complexity of the system. Simple probabilistic methods could be examined for situations where no trip buffer is matched for the current trip.

Improvements in the Path Maker application could be developed to delete redundant trip data and store the best or most ideal path a user traverses. For example, if the user travels home from work everyday at 5 o'clock, there is no benefit to creating and storing a buffer for each of these redundant trips. In an effort to streamline the operations further and provide additional scalability, the polygons representing the trips could be approximated using bounding boxes. This would reduce the complexity of the GIS operations used to determine whether a line lies within a particular area. For the applications mentioned, such as traffic incident detection, the reduction in accuracy of the approach may not impact the performance of the system.

Future research should also examine linking TRAC-IT to 511 or other forms of real-time advanced traveler information systems to push information to the traveler based on where they are currently and where they are likely to be going. Such real-time information could include traffic incidents, dynamic congestion pricing, managed lane pricing, highly-targeted commercial advertising (when appropriate), transit scheduling changes, and other useful data. Now that Navteq has purchased Traffic.com, it may be possible to re-open a dialog with Traffic.com to test TRAC-IT using real-time speed sensors on Tampa Bay highways. Alternately, other cities with different types of advanced traveler information systems in areas such as South Florida could be evaluated for the possibility of linking those systems to TRAC-IT. Future enhancements for incident detections and alerts could include relaying driving directions for alternate routes and verbal feedback to the user as an improvement over the current text message that simply alerts the user of an incident. Since TRAC-IT is not restricted to a vehicle, such information could reach individuals before they travel on multiple modes of transportation such as walking, biking, and transit. 
The applications of TRAC-IT are not limited to travel behavior change. This tool could help evaluate the travel patterns of various market segments or policy decisions. For example, TRAC-IT could be used to evaluate the impacts of various land use proposals such as transit-oriented development on transit use, greenhouse gases, and mobility. TRAC-IT provides a simple method to collect any type of location-based data, and therefore linking mobile phones to alternate types of devices, such as carbon-dioxide sensors, or vehicles to collect other types of data via the mobile phone may be useful. 


\section{References}

(1) Cleland, F., and P. Winters (1999) "Reducing vehicle trips and vehicle miles of travel through customized travel options, final report: results of survey and conclusions". Center for Urban Transportation Research, College of Engineering, University of South Florida. Florida Department of Transportation. http://www.cutr.usf.edu/tdm/pdf/reducing VMT.pdf, accessed September 17, 2007

(2) Winters, P., N. Georggi, and S. Barbeau (2005) "Traveling Smart: Increasing Transit Ridership through Automated Collection (TRAC) of Individual Travel Behavior Data and Personalized Feedback". National Center for Transit Research, Center for Urban Transportation Research, University of South Florida. Florida Department of Transportation. http://www.nctr.usf.edu/pdf/576-16.pdf, accessed September 17, 2007.

(3) O'Fallon, Carolyn, and Charles Sullivan. Personalised Marketing - Improving Evaluation. Dr 26th Australasian Transport Research Forum Wellington, New Zealand. 1-3 October 2003

(4) Richardson, A.J. (2003) "Temporal variability of car usage as an input for the design of Before \& After studies," paper presented at the 82nd Annual Meeting of the Transportation Research Board, Washington, D.C.

(5) Pendyala, R.M., A. Parashar and G.R. Muthyalagari (2000) "Measuring day-to day variability in travel characteristics using GPS data," 80th Annual Meeting of the Transportation Research Board, January 7-11, 2001 Washington.

(6) Schlich, Robert (2001) "Measurement issues in identifying variability in travel behavior, " 1st Swiss Transport Research Conference Monte Verità, Ascona. Accessed October 2007 at http://www.strc.ch/schlich.pdf.

(7) Socialdata Australia Pty Ltd, "Individualized Marketing: Reducing Car Kilometers - A Global Approach," Institute for Transport and Infrastructure Research at http://www.aspoaustralia.org.au/References/Berlin\%20Abstract\%20Socialdata\%20IndiMark.pdf. Last Accessed 2/28/2008.

(8) TravelSmart, Australia at http://www.travelsmart.gov.au/training/packaging comm indi.html. Last Accessed 2/28/2008.

(9) Oregon Department of Transportation Individual Transportation Options Pilot Project Final Report of IndiMark $®$ and Behavioral Analysis For the Cities of Salem-Keizer, Eugene, and Bend, Oregon March 2007 http://www.cherriots.org/Documents/TravelSmart final\%20report.pdf. Last Accessed 2/28/2008.

(10) MELE Associates, Inc. (2006), "Individualized Marketing Demonstration Program Individual City Report for Durham, NC," Federal Transit Administration. Accessed September 21, 2007 at http://www.fta.dot.gov/documents/Durham Report Final.pdf

(11) MELE Associates, Inc. (2006), "Individualized Marketing Demonstration Program Individual City Report for Sacramento, CA," Federal Transit Administration. Accessed September 21, 2007 at http://www.fta.dot.gov/documents/Sacramento Report Final.pdf 
(12) MELE Associates, Inc. (2006), Individualized Marketing Demonstration Program

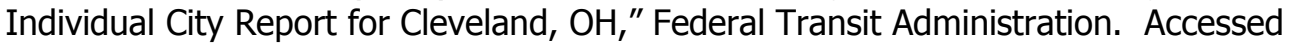
September 21, 2007 at http://www.fta.dot.gov/documents/Cleveland Report Final.pdf

(13) MELE Associates, Inc. (2006), Individualized Marketing Demonstration Program Individual City Report for Bellingham, WA," Federal Transit Administration. Accessed September 21, 2007 at http://www.fta.dot.gov/documents/Bellingham Report Final.pdf

(14) Rose, G., and E. Ampt (2001) "Travel blending: an Australian travel awareness initiative," Transportation Research Part D volume 6, issue 2 pages 95-110

(15) Personalized travel planning: evaluation of 14 pilots part funded by U.K. Department for Transport. Accessed September 2007 at http://www.dft.gov.uk/pgr/sustainable/travelplans/ptp/personalisedtravelplanningev577 4?page $=6 \#$ a1019

(16) Fujii, S. and Taniguchi, A. (2003) "Reducing family car use by providing travel advice or by requesting behavioral plans: an experimental analysis of travel feedback programs," 10th International Conference on Travel Behavior Research, Lucerne, August 2003

(17) Microsoft. "Transact-SQL Reference (Transact-SQL)," SQL Server 2005 Books Online, September 2007. http://msdn2.microsoft.com/en-us/library/ms189826.aspx. Last Accessed 2/27/2008.

(18) Oracle. "Database $11 \mathrm{~g}$ | Oracle Database $11 \mathrm{~g} \mid$ Oracle," http://www.oracle.com/database/index.html. Last Accessed 2/27/2008.

(19) PostgreSQL. "PostgreSQL: The world's most advanced open source database," http://www.postgresql.org/. (c) 1996-2007 PostgresSQL Global Development Group. Last Accessed 2/27/2008.

(20) Microsoft. "Spatial Sample," SQL Server 2005 Books Online, September 2007. http://msdn2.microsoft.com/en-us/library/ms345264.aspx. Last Accessed 2/27/2008.

(21) Microsoft. "How to: Debug T-SQL Using the SQL Server Management Studio," MSDN Library. http://msdn2.microsoft.com/en-us/library/ms241871(VS.80).aspx. (C) 2008 Microsoft Corporation. Last Accessed 2/27/2008.

(22) Oracle. "Oracle Spatial \& Oracle Locator," http://www.oracle.com/database/spatial.html. Last Accessed 2/27/2008.

(23) PostGIS. "What is PostGIS?" http://postgis.refractions.net/. Last Accessed 2/27/2008.

(24) Microsoft. ".NET Framework Developer Center," http://msdn2.microsoft.com/enus/netframework/default.aspx. (C) 2008 Microsoft Corporation. Last Accessed 2/27/2008.

(25) Microsoft. "Microsoft VisualStudio," http://msdn2.microsoft.com/enus/vstudio/default.aspx. (c) Microsoft Corporation. Last Accessed 2/27/2008.

(26) Microsoft. "Microsoft SQL Server 2005 Home," http://www.microsoft.com/sql/default.mspx. Last Accessed 2/28/2008.

(27) ABI Research. "GPS-enabled Location-Based Services (LBS) Subscribers Will Total 315 Million in Five Years," New York, September 27, 2006.

http://www.abiresearch.com/abiprdisplay.jsp?pressid=731. Last Accessed 2/28/2008.

(28) ABI Research, "Personal Locator Services to Reach More than 20 Million North American Consumers by 2011," New York, November 28th, 2006.

http://www.abiresearch.com/abiprdisplay.jsp?pressid=766. Last Accessed 2/28/2008. 
(29) Simmons, R., B. Browning, Yilu Zhang and V. Sadekar. (2006) "Learning to Predict Driver Route and Destination Intent," Proceedings. 2006 IEE Intelligent Transportation Systems, Toronto, Canada, 2006

(30) Karbassi, A., and M. Barth, (2003) "Vehicle Route Prediction and Time of Arrival Estimation Techniques for Improved Transportation System Management." In Proceedings of the Intelligent Vehicles Symposium: p. 511-516

(31) Krumm, John, and Eric Horvitz (2006) "Predestination: Inferring Destinations from Partial Trajectories", UbiComp 2006: The Eighth International Conference on Ubiquitous Computing, September 2006.

(32) Marmasse, N., and C. Schmandt, (2002) "A User-Centered Location Model". Personal and Ubiquitous Computing, (6): p. 318-321.

(33) Ashbrook, D., and T. Starner, (2003) "Using GPS To Learn Significant Locations and Predict Movement Across Multiple Users." Personal and Ubiquitous Computing, 7(5): p. 275-286

(34) Hariharan, R., and K. Toyama. (2004) "Project Lachesis: Parsing and Modeling Location Histories". In Geographic Information Science: Third International Conference, GIScience. Adelphi, MD, USA: Springer-Verlag GmbH.

(35) Liao, L., D. Fox, and H. Kautz. (2004) "Learning and Inferring Transportation Routines in Proceedings of the 19th National Conference on Artificial Intelligence" (AAAI 2004). San Jose, CA, USA.

(36) Krumm, John and Eric Horvitz (2006) "Predestination: Inferring Destinations from Partial Trajectories", UbiComp 2006: The Eighth International Conference on Ubiquitous Computing, September 2006.

(37) ESRI. "ArcGIS: The Complete Enterprise System," http://www.esri.com/software/arcgis/index.html. (C) 1995-2008 ESRI. Last Accessed 2/28/2008.

(38) ESRI. "ArcGIS Server," http://www.esri.com/software/arcgis/index.html. (C) 1995-2008 ESRI. Last Accessed 2/28/2008.

(39) ESRI. "ArcView Key Features," http://www.esri.com/software/arcgis/index.html. (C) 1995-2008 ESRI. Last Accessed 2/28/2008. 